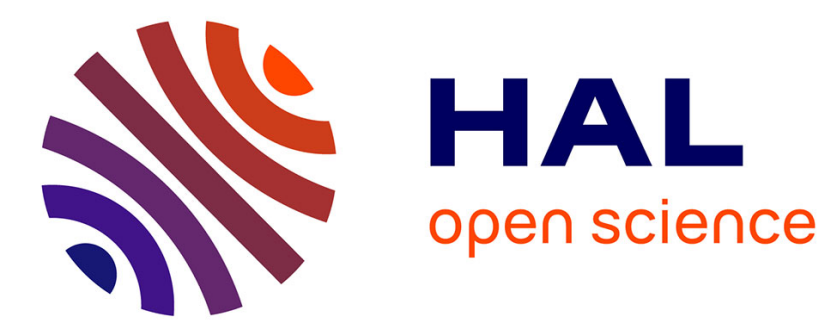

\title{
Shape sensitivity analysis for elastic structures with generalized impedance boundary conditions of the Wentzell type -Application to compliance minimization
}

Fabien Caubet, Djalil Kateb, Frédérique Le Louër

\section{- To cite this version:}

Fabien Caubet, Djalil Kateb, Frédérique Le Louër. Shape sensitivity analysis for elastic structures with generalized impedance boundary conditions of the Wentzell type -Application to compliance minimization. Journal of Elasticity, In press, Journal of Elasticity. hal-01525249

HAL Id: hal-01525249

https://hal.science/hal-01525249

Submitted on 19 May 2017

HAL is a multi-disciplinary open access archive for the deposit and dissemination of scientific research documents, whether they are published or not. The documents may come from teaching and research institutions in France or abroad, or from public or private research centers.
L'archive ouverte pluridisciplinaire HAL, est destinée au dépôt et à la diffusion de documents scientifiques de niveau recherche, publiés ou non, émanant des établissements d'enseignement et de recherche français ou étrangers, des laboratoires publics ou privés. 


\title{
Shape sensitivity analysis for elastic structures with generalized impedance boundary conditions of the Wentzell type - Application to compliance minimization
}

\author{
Fabien Caubet*1, Djalil Kateb ${ }^{\dagger 2}$, and Frédérique Le Louër ${ }^{\ddagger 2}$ \\ ${ }^{1}$ Institut de Mathématiques de Toulouse, Université de Toulouse, F-31062 Toulouse Cedex 9, France. \\ ${ }^{2}$ Sorbonne Universités, Université de Technologie de Compiègne, LMAC EA2222 Laboratoire de \\ Mathématiques Appliquées de Compiègne - CS 60319 - 60203 Compiègne cedex, France
}

May 19, 2017

\begin{abstract}
This paper focuses on Generalized Impedance Boundary Conditions (GIBC) with second order derivatives in the context of linear elasticity and general curved interfaces. A condition of the Wentzell type modeling thin layer coatings on some elastic structure is obtained through an asymptotic analysis of order one of the transmission problem at the thin layer interfaces with respect to the thickness parameter. We prove the well-posedness of the approximate problem and the theoretical quadratic accuracy of the boundary conditions. Then we perform a shape sensitivity analysis of the GIBC model in order to study a shape optimization/optimal design problem. We prove the existence and characterize the first shape derivative of this model. A comparison with the asymptotic expansion of the first shape derivative associated to the original thin layer transmission problem shows that we can interchange the asymptotic and shape derivative analysis. Finally we apply these results to the compliance minimization problem. We compute the shape derivative of the compliance in this context and present some numerical simulations.
\end{abstract}

Keywords: asymptotic analysis, generalized impedance boundary conditions, Wentzell conditions, shape calculus, shape sensitivity analysis, compliance minimization, linear elasticity.

AMS Classification: 35C20, 49Q10, 49Q12, 74B05, 74P05

\section{Introduction and main results}

\subsection{Introduction and general notations}

This paper deals with the mathematical analysis and the shape optimization of some elastic structures coated with a very thin layer of constant thickness. Our objective is first to construct a model problem where the thin layer effects are characterized by perturbed boundary conditions with second order derivatives called boundary condition of the Wentzell type. Then we aim to make a shape sensitivity analysis for this reduced problem using shape calculus tools. These subjects find applications in various area such as elastographic imaging and structural optimal design.

On the one hand, the theoretical and numerical analysis of a thin structure could be a very complex work. Hence, in order to overcome this difficulty, a classical strategy is to approximate an original structure with a thin layer (see, e.g., 10, 30, 46, 16, ) or a rough boundary (see, e.g., 36, 1])

\footnotetext{
*fabien.caubet@math.univ-toulouse.fr

†djalil.kateb@utc.fr

‡frederique.le-louer@utc.fr
} 
by another domain with new boundary conditions, called Generalized Impedance Boundary Conditions (GIBC). The GIBC contain some informations on the geometry and the material characteristics of the thin structure. For exterior problems, artificial (or approximate) conditions are another example (see, e.g., [25, 29]). These boundary conditions are generally simple differential conditions obtained from an asymptotic analysis of the full elliptic equations in the thin layer with respect to a characteristic length: the thickness of the layer, the scale of the roughness, the diameter of the artificial boundary, for the previous three examples, respectively. In linear elasticity, it deserves to mention the book of Ciarlet 21, where a local representation of the GIBC is proposed, and others works [26, 11] in the context of thin elastic plates or shells. The construction of GIBC is also treated for example by Antoine et al [9], by Poignard [44] or by Haddar et al [31, 32, 18] in acoustics and electromagnetism for general three-dimensional surfaces.

On the other hand, the problem of finding an optimal shape for physical issues described by elliptic boundary value problems is widely studied from many years. In linear elasticity, the idea is to study the influence of the shape of a structure on its behavior as for example its rigidity. To this end, several methods can be employed as the classical geometrical shape optimization approach (see, e.g., the work of Murat et al. [39]), the homogenization method (see, e.g., the work of Allaire et al. [5), the topological shape optimization approach (see, e.g., the work of Garreau et al. 28]) or the so-called level-set method (see, e.g., the works of Allaire et al. [7]). Several works deal about the problem of minimizing the compliance of the structure where standard boundary conditions are imposed on the free boundary. We can here mention the recent works of Allaire et al. [6], Amstutz et al. [8], Novotny et al. [4] and Dambrine et al. 24]. This list of references is far from being exhaustive and we also refer to the books of Sokolowski et al. [45], of Henrot et al. [35], of Allaire [3, 4] and of Haslinger et al. 33] for background notions about shape optimization methods.

Finally, more recently, one can find new advances on the computation of shape derivatives and/or the solution of shape optimization and inverse obstacle scattering problems when a GIBC of the first and second orders are imposed on the unknown boundary. For example, we can mention the works of Cakoni et al. 15, 14 and Caubet et al. 17] for the Laplace's equation, of Bourgeois et al. [12] and Kateb et al. [37] for the Helmholtz equation and of Chaulet et al. [19] for the Maxwell's equations.

The present paper is dedicated to the shape sensitivity analysis when first-order GIBC are imposed on the free boundary of some elastic structures.

General notations. For a smooth bounded open set $\omega$ of $\mathbb{R}^{d}(d \geq 2)$ with a boundary $\Gamma$, we denote by $\mathrm{H}^{s}(\omega)$ and $\mathrm{H}^{s}(\Gamma)$ the standard complex valued, Hilbert-Sobolev spaces of order $s \in \mathbb{R}$ defined on $\omega$ and $\Gamma$ respectively (with the convention $\mathrm{H}^{0}=\mathrm{L}^{2}$ ). Spaces of vector functions will be denoted by boldface letters, thus $\mathbf{H}^{s}=\left(\mathrm{H}^{s}\right)^{d}$. Moreover the tangential gradient is denoted by $\nabla_{\Gamma}$ and the surface divergence is denoted by $\operatorname{div}_{\Gamma}$. For any vector field $\boldsymbol{u}, \nabla_{\Gamma} \boldsymbol{u}$ is the matrix whose the $j$-th column is the tangential gradient of the $j$-th component of $\boldsymbol{u}$. For any matrix-valued function $\mathcal{F}, \operatorname{div}_{\Gamma} \mathcal{F}$ is the vector whose the $j$-th component is the surface divergence of the $j$-th column of $\mathcal{F}$. We denote by $\mathrm{I}_{d}$ the $d \times d$ identity matrix. Finally, notice that for two matrices $A$ and $B$, we denote by $A: B$ the matrix scalar product between $A$ and $B$.

\subsection{Setting of the problem, main results and organization of the paper}

Introduction of the two considered problems. Let $\Omega$ be a Lipschitz bounded open set of $\mathbb{R}^{d}$, where $d \geq 2$ is an integer representing the dimension. We assume that the solid $\Omega$ consists of an isotropic material with a linear behavior. The boundary of $\Omega$ is such that $\partial \Omega=: \Gamma_{D} \cup \Gamma_{N}$ where $\Gamma_{D}$ and $\Gamma_{N}$ are two non-empty open sets of $\partial \Omega$ and $\left|\Gamma_{D}\right|>0$. Let $\delta>0$ be a fixed (small) real number and let us define the following set of admissible shapes:

$$
\begin{array}{r}
\mathcal{O}_{\delta}:=\left\{\omega \subset \subset \Omega \text { be a smooth (at least } C^{2}\right) \text { open set such that } \mathrm{d}(x, \partial \Omega)>\delta \text { for all } x \in \omega \\
\text { and such that } \Omega \backslash \bar{\omega} \text { is connected }\} .
\end{array}
$$

Let us consider a (nonempty) inclusion $\omega \in \mathcal{O}_{\delta}$ with boundary $\partial \omega=: \Gamma$. Then $\Omega \backslash \bar{\omega}$ represents a reference configuration of an elastic solid assumed to be built on $\Gamma_{D}$. We denote by $\mathbf{n}$ the unit 
normal vector to $\partial \Omega$ and $\Gamma$ directed outward to $\Omega \backslash \bar{\omega}$. Moreover, H represents the mean curvature of $\Gamma$ and $b$ is the signed distance.

We denote by $A_{\text {ext }}$ the Hooke's law defined, for any symmetric matrix $\xi$, by

$$
A_{\text {ext }} \xi:=2 \mu_{\text {ext }} \xi+\lambda_{\text {ext }} \operatorname{Tr}(\xi) \mathrm{I}_{d},
$$

where $\mu_{\text {ext }}>0$ and $\lambda_{\text {ext }}>0$ are two positive constants which represent the Lamé coefficients of the material making up the solid and we introduce, for any $\boldsymbol{u} \in \mathbf{H}^{1}(\Omega)$, the symmetrized gradient

$$
e(\boldsymbol{u}):=\frac{1}{2}\left(\nabla \boldsymbol{u}+{ }^{t} \nabla \boldsymbol{u}\right)
$$

Let $\varepsilon>0$. We consider that $\Gamma$ has an interior thin layer with thickness $\varepsilon$ surrounding $\omega$ defined by

$$
\omega_{\text {int }}^{\varepsilon}:=\{x+s \mathbf{n}(x) \mid x \in \Gamma \text { and } 0<s<\varepsilon\} .
$$

We recall that the normal vector $\mathbf{n}$ is directed inward the inclusion $\omega$. We set $\omega^{\varepsilon}:=\omega \backslash \overline{\omega_{\text {int }}^{\varepsilon}}$ and we denote its boundary by $\Gamma^{\varepsilon}$. We also denote by $\mathbf{n}^{\varepsilon}$ the inward unit normal vector to $\Gamma^{\varepsilon}$. In the sequel, we use the lower index $e$ for all quantities related to $\Omega \backslash \bar{\omega}$ and the lower index $i$ for all quantities related to $\omega_{\text {int }}^{\varepsilon}$.

We introduce the following Sobolev space

$$
\mathbf{H}_{\Gamma_{D}}^{1}(\Omega \backslash \bar{\omega}):=\left\{\boldsymbol{v} \in \mathbf{H}^{1}(\Omega \backslash \bar{\omega}) ; \boldsymbol{v}=\mathbf{0} \text { on } \Gamma_{D}\right\} .
$$

Let $\boldsymbol{f} \in \mathbf{L}^{2}(\Omega \backslash \bar{\omega})$ be some exterior forces and a load $\boldsymbol{g} \in \mathbf{H}^{-1 / 2}\left(\Gamma_{N}\right)$. We are concerned with the following transmission problem

$$
\left\{\begin{aligned}
-\operatorname{div} A_{\text {ext }} e\left(\boldsymbol{u}_{e}^{\varepsilon}\right) & =\boldsymbol{f} & & \text { in } \Omega \backslash \bar{\omega} \\
-\operatorname{div} A_{\text {int }} e\left(\boldsymbol{u}_{i}^{\varepsilon}\right) & =\mathbf{0} & & \text { in } \omega_{\text {int }}^{\varepsilon} \\
\boldsymbol{u}_{e}^{\varepsilon} & =\mathbf{0} & & \text { on } \Gamma_{D} \\
\left(A_{\mathrm{ext}} e\left(\boldsymbol{u}_{e}^{\varepsilon}\right)\right) \mathbf{n} & =\boldsymbol{g} & & \text { on } \Gamma_{N} \\
\left(A_{\text {int }} e\left(\boldsymbol{u}_{i}^{\varepsilon}\right)\right) \mathbf{n} & =\left(A_{\text {ext }} e\left(\boldsymbol{u}_{e}^{\varepsilon}\right)\right) \mathbf{n} & & \text { on } \Gamma \\
\boldsymbol{u}_{i}^{\varepsilon} & =\boldsymbol{u}_{e}^{\varepsilon} & & \text { on } \Gamma \\
A_{\text {int }} e\left(\boldsymbol{u}_{i}^{\varepsilon}\right) \mathbf{n}^{\varepsilon} & =\mathbf{0} & & \text { on } \Gamma^{\varepsilon}
\end{aligned}\right.
$$

where $A_{\text {int }}$ represents the Hooke's law associated to $\omega_{\text {int }}^{\varepsilon}$ with Lamé coefficients $\mu_{\text {int }}>0$ and $\lambda_{\text {int }}>0$. The solution of such a problem exists, is unique and belongs to $\mathbf{H}_{\Gamma_{D}}^{1}\left(\Omega \backslash \bar{\omega} \cup \omega_{\text {int }}^{\varepsilon}\right)$ thanks to the Lax-Milgram theorem and the Korn's inequality.

We introduce the following Hilbert space

$$
\mathbf{V}(\Gamma)=\left\{\boldsymbol{\psi} \in \mathbf{L}^{2}(\Gamma) ; e_{\Gamma}(\boldsymbol{\psi}) \in \mathbf{L}^{2}(\Gamma)\right\}
$$

endowed with the graph norm $\|\boldsymbol{\psi}\|_{\mathbf{V}(\Gamma)}^{2}:=\left(\|\boldsymbol{\psi}\|_{\mathbf{L}^{2}(\Gamma)}^{2}+\left\|e_{\Gamma}(\boldsymbol{\psi})\right\|_{\mathbf{L}^{2}(\Gamma)}^{2}\right)^{1 / 2}$ where

$$
e_{\Gamma}(\boldsymbol{\psi}):=\frac{1}{2} \Pi_{d}\left(\nabla_{\Gamma} \boldsymbol{\psi}+{ }^{t} \nabla_{\Gamma} \boldsymbol{\psi}\right) \Pi_{d} \quad \text { and } \quad \Pi_{d}:=\mathrm{I}_{d}-\mathbf{n} \otimes \mathbf{n}
$$

We denote its dual space by $\mathbf{V}^{\prime}(\Gamma)$. Then, we set

$$
\mathcal{H}(\Omega \backslash \bar{\omega}):=\left\{\boldsymbol{v} \in \mathbf{H}_{\Gamma_{D}}^{1}(\Omega \backslash \bar{\omega}) ; \boldsymbol{v}_{\mid \Gamma} \in \mathbf{V}(\Gamma)\right\}
$$

The space $\mathcal{H}(\Omega \backslash \bar{\omega})$ endowed with the graph norm $\|\boldsymbol{v}\|_{\mathcal{H}(\Omega \backslash \bar{\omega})}:=\left(\|\boldsymbol{v}\|_{\mathbf{H}^{1}(\Omega \backslash \bar{\omega})}^{2}+\|\boldsymbol{v}\|_{\mathbf{V}(\Gamma)}^{2}\right)^{1 / 2}$ is a Hilbert space.

We prove below (see Proposition 2.1) that the solution of the transmission problem $(1.2)$ can be approximated using an asymptotic analysis by the solution $\boldsymbol{u} \in \mathcal{H}(\Omega \backslash \bar{\omega})$ of the following problem with Generalized Impedance Boundary Conditions (GIBC) on $\Gamma$ :

$$
\left\{\begin{aligned}
-\operatorname{div}\left(A_{\mathrm{ext}} e(\boldsymbol{u})\right) & =\boldsymbol{f} & & \text { in } \Omega \backslash \bar{\omega} \\
\boldsymbol{u} & =\mathbf{0} & & \text { on } \Gamma_{D} \\
\left(A_{\mathrm{ext}} e(\boldsymbol{u})\right) \mathbf{n} & =\boldsymbol{g} & & \text { on } \Gamma_{N} \\
\left(A_{\mathrm{ext}} e(\boldsymbol{u})\right) \mathbf{n}-\varepsilon \operatorname{div}_{\Gamma}\left(\sigma_{\Gamma}(\boldsymbol{u})\right) & =\mathbf{0} & & \text { on } \Gamma .
\end{aligned}\right.
$$


Here, we have used the following notations:

$$
\begin{aligned}
\sigma_{\Gamma}(\boldsymbol{u}) & :=\bar{\lambda}_{\text {int }}\left(\operatorname{div}_{\Gamma} \boldsymbol{u}\right) \Pi_{d}+2 \mu_{\mathrm{int}} e_{\Gamma}(\boldsymbol{u})=\bar{A}_{\mathrm{int}} e_{\Gamma}(\boldsymbol{u}) \\
\bar{A}_{\text {int }} \xi & :=2 \mu_{\mathrm{int}} \xi+\bar{\lambda}_{\mathrm{int}} \operatorname{Tr}(\xi) \Pi_{d}
\end{aligned}
$$

where $\mu_{\mathrm{int}}$ and $\bar{\lambda}_{\mathrm{int}}:=\frac{2 \lambda_{\mathrm{int}} \mu_{\mathrm{int}}}{\lambda_{\mathrm{int}}+2 \mu_{\mathrm{int}}}$ are the modified Lamé constants of the thin layer. The wellposedness of this problem is studied in Appendix A (see Proposition A.1).

Remark 1.1. (i) The negative Wentzell-type operator $\boldsymbol{\psi} \mapsto-\operatorname{div}_{\Gamma}\left(\sigma_{\Gamma}(\boldsymbol{\psi})\right)$ is a positive symmetric operator bounded from $\mathbf{V}(\Gamma)$ to $\mathbf{V}^{\prime}(\Gamma)$. Indeed we have

$$
\begin{aligned}
\left\langle-\operatorname{div}_{\Gamma}\left(\sigma_{\Gamma}(\boldsymbol{\psi})\right), \boldsymbol{\varphi}\right\rangle_{\mathbf{V}^{\prime}(\Gamma), \mathbf{V}(\Gamma)} & =\int_{\Gamma} \bar{\lambda}_{\operatorname{int}}\left(\operatorname{div}_{\Gamma} \boldsymbol{\psi}\right)\left(\operatorname{div}_{\Gamma} \boldsymbol{\varphi}\right) d s+2 \mu_{\mathrm{int}} \int_{\Gamma} e_{\Gamma}(\boldsymbol{\psi}): e_{\Gamma}(\boldsymbol{\varphi}) \\
& =\left\langle\boldsymbol{\psi},-\operatorname{div}_{\Gamma}\left(\sigma_{\Gamma}(\boldsymbol{\varphi})\right)\right\rangle_{\mathbf{V}(\Gamma), \mathbf{V}^{\prime}(\Gamma)} .
\end{aligned}
$$

(ii) For any $\boldsymbol{\psi} \in \mathbf{L}^{2}(\Gamma)$, let set $\boldsymbol{\psi}_{\mathrm{n}}:=\boldsymbol{\psi} \cdot \mathbf{n}$ and $\boldsymbol{\psi}_{\mathrm{t}}:=\boldsymbol{\psi}-\boldsymbol{\psi}_{\mathrm{n}} \mathbf{n}$. Then we get the following inclusion $\left\{\boldsymbol{\psi} \in \mathbf{L}^{2}(\Gamma) ; \boldsymbol{\psi}_{\mathrm{t}} \in \mathbf{H}^{1}(\Gamma)\right\} \subset \mathbf{V}(\Gamma)$ thanks to the equality

$$
e_{\Gamma}(\boldsymbol{\psi})=e_{\Gamma}\left(\boldsymbol{\psi}_{\mathrm{t}}\right)+\boldsymbol{\psi}_{\mathrm{n}}\left[\mathrm{D}^{2} b\right] .
$$

Moreover, using the formulas given in [21, page 88], one can check that the operator $\boldsymbol{\psi} \in$ $\mathbf{V}(\Gamma) \mapsto e_{\Gamma}(\boldsymbol{\psi}) \in \mathbf{L}^{2}(\Gamma)$ corresponds to the operator $\gamma_{\alpha \beta}$ defined in [21, Theorem 2.7.1]. Using an atlas for the boundary $\Gamma$, one can derive an inequality of Korn-type on 2-dimensional compact manifolds without boundary from [21, Theorem 2.7.1] ensuring that there exists a constant $c_{0}>0$ depending on $\Gamma$ such that

$$
\left(\left\|\boldsymbol{\psi}_{\mathrm{t}}\right\|_{\mathbf{H}^{1}(\Gamma)}^{2}+\left\|\boldsymbol{\psi}_{\mathrm{n}}\right\|_{\mathrm{L}^{2}(\Gamma)}^{2}\right)^{1 / 2} \leq c_{0}\|\boldsymbol{\psi}\|_{\mathbf{V}(\Gamma)}
$$

In other words, the two spaces coincide, i.e.

$$
\left\{\boldsymbol{\psi} \in \mathbf{L}^{2}(\Gamma) ; \boldsymbol{\psi}_{\mathrm{t}} \in \mathbf{H}^{1}(\Gamma)\right\}=\mathbf{V}(\Gamma)
$$

We summarize the notations concerning the domains in Figure 1 below.

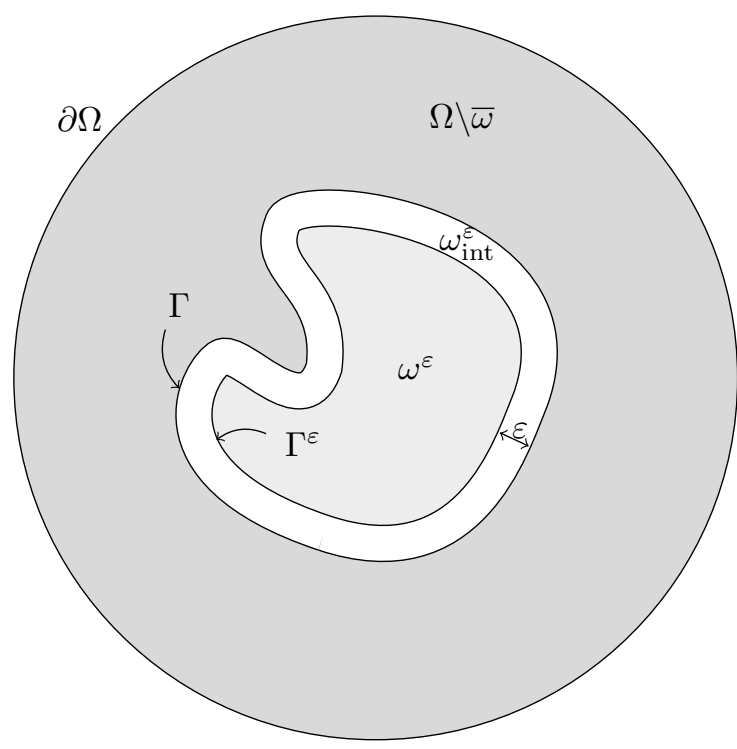

(a)

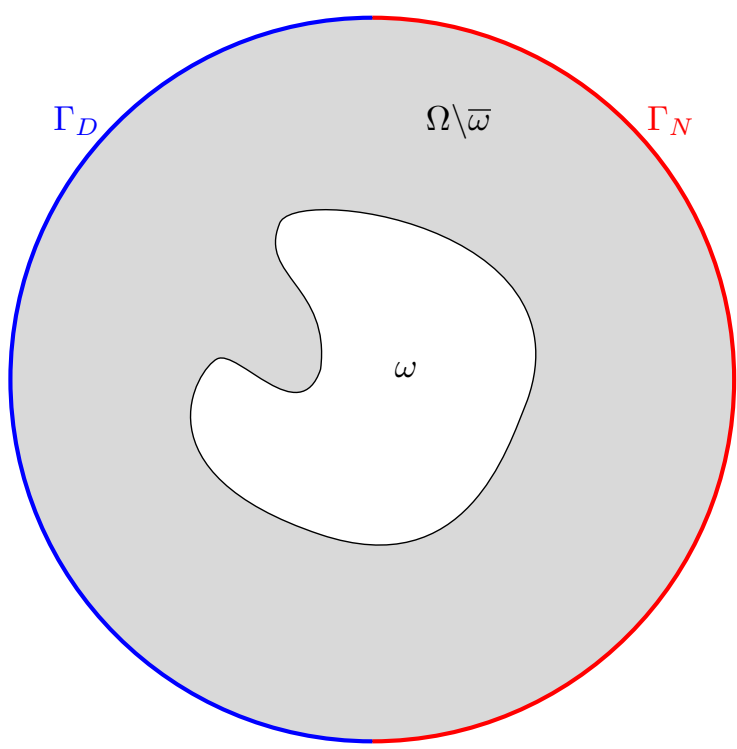

(b)

Figure 1: Notations for the thin layer model (left) and the obtained GIBC model (right). 
Main aims and results of the paper. The first aim of this work is to derive the GIBC on $\Gamma$ given in Problem (1.4). To do this, we perform an asymptotic analysis on the transmission problem (1.2) with respect to the thickness of the layer. We then obtain the following main result of this paper which gives the rate of convergence of this analysis. This part is treated in Section 2 and uses asymptotic results given in Appendix B

Theorem 1.2. If $\boldsymbol{f} \in \mathbf{H}^{1 / 2}(\Omega \backslash \bar{\omega})$, then there exists a constant $C$, depending only on the domains $\Omega$ and $\omega$, such that

$$
\left\|\boldsymbol{u}-\boldsymbol{u}_{e}^{\varepsilon}\right\|_{\mathbf{H}^{1}(\Omega \backslash \bar{\omega})} \leq C \varepsilon^{2},
$$

where $\boldsymbol{u} \in \mathcal{H}(\Omega \backslash \bar{\omega})$ is the solution of Problem 1.4 and $\boldsymbol{u}_{e}^{\varepsilon} \in \mathbf{H}_{\Gamma_{D}}^{1}(\Omega \backslash \bar{\omega})$ is defined by Problem (1.2).

The second main aim is to make a shape sensitivity analysis of this problem. Hence we prove the existence of shape derivatives with respect to the shape $\omega$ and characterize the shape derivative of Problem 1.4 (we refer to Section 3.2 for some reminders about the definition of the shape derivative). Then we obtain the following second main result.

Theorem 1.3. Let $\boldsymbol{V} \in\left\{\boldsymbol{\theta} \in \boldsymbol{C}^{2, \infty}=\boldsymbol{C}^{2} \cap \mathbf{W}^{2, \infty}\left(\mathbb{R}^{d}\right) ; \operatorname{supp}(\boldsymbol{\theta}) \subset \overline{\Omega_{\delta}} ;\|\boldsymbol{\theta}\|_{2, \infty}<\min \left(\frac{\delta}{3}, 1\right)\right\}$ and let $\boldsymbol{u} \in \mathcal{H}(\Omega \backslash \bar{\omega})$ the solution of Problem (1.4). The shape derivative $\boldsymbol{u}^{\prime}$ of $\boldsymbol{u}$, which belongs to $\mathbf{L}^{2}(\Omega \backslash \bar{\omega})$, exists. Moreover, if $\boldsymbol{u} \in \mathbf{H}^{2}\left(\overline{\Gamma)}\right.$, then $\boldsymbol{u}^{\prime} \in \mathcal{H}(\Omega \backslash \bar{\omega})$ is the only solution of the following boundary value problem

$$
\left\{\begin{aligned}
-\operatorname{div}\left(A_{\text {ext }} e\left(\boldsymbol{u}^{\prime}\right)\right) & =\mathbf{0} & & \text { in } \Omega \backslash \bar{\omega} \\
\boldsymbol{u}^{\prime} & =\mathbf{0} & & \text { on } \Gamma_{D} \\
\left(A_{\text {ext }} e\left(\boldsymbol{u}^{\prime}\right)\right) \mathbf{n} & =\mathbf{0} & & \text { on } \Gamma_{N} \\
\left(A_{\text {ext }} e\left(\boldsymbol{u}^{\prime}\right)\right) \mathbf{n}-\varepsilon \operatorname{div}_{\Gamma}\left(\sigma_{\Gamma}\left(\boldsymbol{u}^{\prime}\right)\right) & =\xi\left(\boldsymbol{u}, V_{\mathrm{n}}\right) & & \text { on } \Gamma,
\end{aligned}\right.
$$

with

$$
\begin{aligned}
\xi\left(\boldsymbol{u}, V_{\mathrm{n}}\right) & :=V_{\mathrm{n}} \boldsymbol{f}+\operatorname{div}_{\Gamma}\left(V_{\mathrm{n}} \Pi_{d}\left(A_{\mathrm{ext}} e(\boldsymbol{u})\right) \Pi_{d}\right)-\varepsilon \operatorname{div}_{\Gamma}\left(V_{\mathrm{n}}\left(\left[\mathrm{D}^{2} b\right]-\mathrm{H} \Pi_{d}\right) \sigma_{\Gamma}(\boldsymbol{u})\right) \\
& -\varepsilon \operatorname{div}_{\Gamma}\left(V_{\mathrm{n}}\left(\bar{A}_{\mathrm{int}} \Pi_{d}\left(\frac{1}{2}\left(\left[\mathrm{D}^{2} b\right] \nabla_{\Gamma} \boldsymbol{u}+{ }^{t}\left(\left[\mathrm{D}^{2} b\right] \nabla_{\Gamma} \boldsymbol{u}\right)\right)\right) \Pi_{d}\right)\right)+\varepsilon \operatorname{div}_{\Gamma}\left(\sigma_{\Gamma}\left(V_{\mathrm{n}} \partial_{\mathbf{n}} \boldsymbol{u}\right)\right) \\
+\varepsilon\left(\left[\mathrm{D}^{2} b\right]\right. & \left.+\mathbf{n} \operatorname{div}_{\Gamma} \Pi_{d}\right)\left(\operatorname{div}_{\Gamma}\left(V_{\mathrm{n}} \sigma_{\Gamma}(\boldsymbol{u})\right)\right)+\varepsilon \operatorname{div}_{\Gamma}\left(\overline{A_{\mathrm{int}}}\left(\frac{1}{2}\left(\left[\nabla_{\Gamma} \boldsymbol{u}\right] \mathbf{n} \otimes \nabla_{\Gamma} V_{\mathrm{n}}+\nabla_{\Gamma} V_{\mathrm{n}} \otimes\left[\nabla_{\Gamma} \boldsymbol{u}\right] \mathbf{n}\right)\right)\right),
\end{aligned}
$$

where $V_{\mathrm{n}}:=\boldsymbol{V} \cdot \mathbf{n}$, where $\mathrm{H}$ is the mean curvature of $\Gamma$ and $b$ is the signed distance.

We also provide an asymptotic analysis on the shape derivatives for the transmission problem 1.2 . and compare it with the shape derivatives of Problem (1.4). This analysis concerning the shape calculus is done in Section 3 .

We conclude this paper with an example of application of this work concerning the minimization of the compliance in Section 4 . We compute the shape derivative of the compliance through the introduction of an adjoint problem and make some numerical simulations in the two-dimensional case in order to illustrate and validate our theoretical results. Thus, defining the compliance of the structure $\Omega \backslash \bar{\omega}$ as

$$
\mathcal{J}(\Omega \backslash \bar{\omega}):=\int_{\Omega \backslash \bar{\omega}} A_{\text {ext }} e(\boldsymbol{u}): e(\boldsymbol{u}),
$$

where $\boldsymbol{u} \in \mathcal{H}(\Omega \backslash \bar{\omega})$ solves Problem (1.4), we obtain the following expression of the shape gradient of this cost functional.

Theorem 1.4. Let $\boldsymbol{V} \in\left\{\boldsymbol{\theta} \in \boldsymbol{C}^{2, \infty}=\boldsymbol{C}^{2} \cap \mathbf{W}^{2, \infty}\left(\mathbb{R}^{d}\right) ; \operatorname{supp}(\boldsymbol{\theta}) \subset \overline{\Omega_{\delta}} ;\|\boldsymbol{\theta}\|_{2, \infty}<\min \left(\frac{\delta}{3}, 1\right)\right\}$. If $\boldsymbol{u} \in \mathbf{H}^{2}(\Gamma)$, then the shape derivative of the cost functional can be written as

$$
\nabla \mathcal{J}(\Omega \backslash \bar{\omega}) \cdot \boldsymbol{V}=\int_{\Gamma} V_{\mathrm{n}} \xi^{*}(\boldsymbol{u}, \boldsymbol{p}+\boldsymbol{u}) d s+\int_{\Gamma} V_{\mathrm{n}}\left(A_{\mathrm{ext}} e(\boldsymbol{u}): e(\boldsymbol{u})\right),
$$


where $\boldsymbol{u} \in \mathcal{H}(\Omega \backslash \bar{\omega})$ is the solution of Problem (1.4) and where

$$
\begin{array}{r}
\xi^{*}(\boldsymbol{u}, \boldsymbol{v}):=-\left(\Pi_{d}\left(A_{\mathrm{ext}} e(\boldsymbol{u})\right) \Pi_{d}\right): \nabla_{\Gamma} \boldsymbol{v}+\varepsilon\left\{\left(\left(\left[\mathrm{D}^{2} b\right]-\mathrm{H} \Pi_{d}\right) \sigma_{\Gamma}(\boldsymbol{u})\right): \nabla_{\Gamma} \boldsymbol{v}+\left(\left[\mathrm{D}^{2} b\right] \nabla_{\Gamma} \boldsymbol{u}\right): \sigma_{\Gamma}(\boldsymbol{v})\right. \\
\left.+\left(\partial_{\mathbf{n}} \boldsymbol{u}\right) \operatorname{div}_{\Gamma}\left(\sigma_{\Gamma}(\boldsymbol{v})\right)+\sigma_{\Gamma}(\boldsymbol{u}):\left(\nabla_{\Gamma}\left(\left[\nabla_{\Gamma} \boldsymbol{v}\right] \mathbf{n}\right)\right)+\operatorname{div}_{\Gamma}\left(\sigma_{\Gamma}(\boldsymbol{v})\left[\nabla_{\Gamma} \boldsymbol{u}\right] \mathbf{n}\right)\right\}+\boldsymbol{f} \cdot \boldsymbol{v}
\end{array}
$$

and $\boldsymbol{p} \in \mathcal{H}(\Omega \backslash \bar{\omega})$ is solution to the following adjoint problem

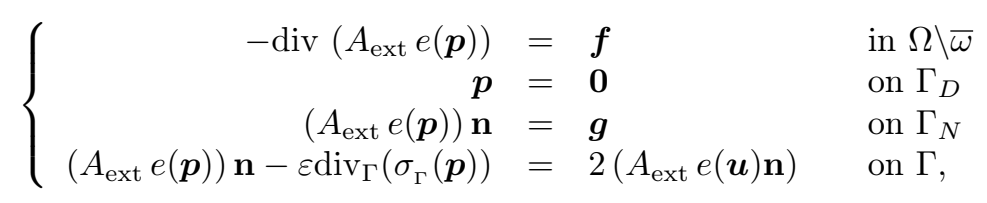

with $V_{\mathrm{n}}:=\boldsymbol{V} \cdot \mathbf{n}$ and $b$ denoting the signed distance.

Remark 1.5. We are confident that the regularity assumption $\boldsymbol{u} \in \mathbf{H}^{2}(\Gamma)$ made in the two previous theorems is useless since automatically satisfied. Unfortunately, up to our knowledge, the arguments to obtain it are not trivial and we postpone this study to a forthcoming work.

\section{Derivation of the GIBC model and convergence analysis}

This section is devoted to the derivation of the GIBC model (1.4) analyzed in this paper and to the proof of Theorem 1.2 To do this, we make an asymptotic analysis with respect to the thin layer of the transmission problem 1.2 .

\subsection{Asymptotic analysis of the transmission problem}

Let $N \in \mathbb{N}$. We want to approximate the solution $\boldsymbol{u}_{e}^{\varepsilon} \in \mathbf{H}_{\Gamma_{D}}^{1}(\Omega \backslash \bar{\omega})$ of the transmission problem 1.2 by the solution $\boldsymbol{v}_{[N]}^{\varepsilon}$ of some boundary value problems of the form

$$
\left\{\begin{array}{rlll}
-\operatorname{div} A_{\mathrm{ext}} e\left(\boldsymbol{v}_{[N]}^{\varepsilon}\right) & =\boldsymbol{f} & \text { in } \Omega \backslash \bar{\omega} \\
\boldsymbol{v}_{[N]}^{\varepsilon} & =\mathbf{0} & & \text { on } \Gamma_{D} \\
\left(A_{\mathrm{ext}} e\left(\boldsymbol{v}_{[N]}^{\varepsilon}\right)\right) \mathbf{n} & =\boldsymbol{g} & & \text { on } \Gamma_{N} \\
C\left(\varepsilon, A_{\mathrm{ext}} e\left(\boldsymbol{v}_{[N]}^{\varepsilon}\right) \mathbf{n}, \boldsymbol{v}_{[N]}^{\varepsilon}\right) & =\mathbf{0} & & \text { on } \Gamma
\end{array}\right.
$$

where $\left\|\boldsymbol{u}_{e}^{\varepsilon}-\boldsymbol{v}_{[N]}^{\varepsilon}\right\|_{\mathbf{H}^{1}(\Omega \backslash \bar{\omega})}=O\left(\varepsilon^{N+1}\right)$ and $C\left(\varepsilon, A_{\text {ext }} e\left(\boldsymbol{v}_{[N]}^{\varepsilon}\right) \mathbf{n}, \boldsymbol{v}_{[N]}^{\varepsilon}\right)$ is a so-called Generalized Impedance Boundary Condition (GIBC). To do so, we follow the procedure described for example in [46, 37]. For any $x \in \Gamma$ and $s \geq 0$, we set $\boldsymbol{u}(x+s \mathbf{n}(x))=: \overline{\boldsymbol{u}}(x, s)$ and we use the change of variables $y=x+s \mathbf{n}(x)=x+\varepsilon S \mathbf{n}(x)$ with $S \in[0,1]$. We set $\overline{\boldsymbol{u}}(x, s)=\overline{\boldsymbol{u}}(x, \varepsilon S)=: \mathbf{U}(x, S)$.

Firstly we obtain the following asymptotic expansion when $\varepsilon \rightarrow 0$ (see Appendix B for details):

$$
\operatorname{div} A_{\text {int }} e(\boldsymbol{u})(x+\varepsilon S \mathbf{n}(x))=\frac{1}{\varepsilon^{2}}\left(\Lambda_{0} \partial_{S}^{2}+\varepsilon \Lambda_{1} \partial_{S}+\sum_{n \geq 2} \varepsilon^{n} \Lambda_{n}\right) \mathbf{U}(x, S),
$$

where

$$
\begin{aligned}
\Lambda_{0} & :=\left(\lambda_{\mathrm{int}}+2 \mu_{\mathrm{int}}\right) \mathbf{n} \otimes \mathbf{n}+\mu_{\mathrm{int}}\left(\mathrm{I}_{d}-\mathbf{n} \otimes \mathbf{n}\right), \\
\Lambda_{1} \mathbf{U} & :=\mu_{\mathrm{int}} \mathrm{H} \boldsymbol{U}+\left(\lambda_{\mathrm{int}}+\mu_{\mathrm{int}}\right)\left(\mathbf{n} \operatorname{div}_{\Gamma} \mathbf{U}+\nabla_{\Gamma}(\mathbf{U} \cdot \mathbf{n})\right), \\
\Lambda_{2} \mathbf{U} & :=\Lambda_{2,2} \mathbf{U}+S \Lambda_{2,1} \partial_{S} \mathbf{U}
\end{aligned}
$$

with

$$
\begin{aligned}
& \Lambda_{2,1} \mathbf{U}:=-\mu_{\mathrm{int}} \operatorname{Tr}\left(\left[\mathrm{D}^{2} b\right]^{2}\right) \mathbf{U}-\left(\lambda_{\mathrm{int}}+\mu_{\mathrm{int}}\right)\left(\mathbf{n}\left(\operatorname{Tr}\left(\left[\mathrm{D}^{2} b\right] \nabla_{\Gamma} \mathbf{U}\right)\right)+\left[\mathrm{D}^{2} b\right] \nabla_{\Gamma}(\mathbf{U} \cdot \mathbf{n})\right), \\
& \left.\Lambda_{2,2} \mathbf{U}:=\left(\lambda_{\mathrm{int}}+\mu_{\mathrm{int}}\right) \nabla_{\Gamma} \operatorname{div}_{\Gamma} \mathbf{U}+\mu_{\mathrm{int}} \Delta_{\Gamma} \mathbf{U}-\lambda_{\mathrm{int}} \mathbf{n}\left(\operatorname{Tr}\left(\left[\mathrm{D}^{2} b\right] \nabla_{\Gamma} \mathbf{U}\right)\right)-\mu_{\mathrm{int}}\left(\left[\mathrm{D}^{2} b\right] \nabla_{\Gamma} \mathbf{U}\right]\right) \mathbf{n} .
\end{aligned}
$$


Moreover the traction trace operator is defined on $\Gamma$, i.e. for $S=0$, by

$$
A_{\text {int }} e(\mathbf{U}):=\frac{1}{\varepsilon} \Lambda_{0} \partial_{S} \mathbf{U}+\lambda_{\operatorname{int}} \mathbf{n} \operatorname{div}_{\Gamma} \mathbf{U}+\mu_{\text {int }}\left[\nabla_{\Gamma} \mathbf{U}\right] \mathbf{n}
$$

and it admits the following expansion on the surface $\Gamma^{\varepsilon}$, i.e. for $S=1$, (see also Appendix $\mathrm{B}$ for details):

$$
A_{\text {int }} e\left(\mathbf{U}_{i}(\cdot, 1)\right)=\frac{1}{\varepsilon} \Lambda_{0} \partial_{S} \mathbf{U}_{i}(\cdot, 1)+\mathbf{B}_{t}^{0} \mathbf{U}_{i}(\cdot, 1)+\sum_{\ell \geq 1} \varepsilon^{\ell} \mathbf{B}_{t}^{\ell} \mathbf{U}_{i}(\cdot, 1),
$$

with

$$
\begin{aligned}
& \mathbf{B}_{t}^{0} \mathbf{U}:=\lambda_{\mathrm{int}} \mathbf{n} \operatorname{div}_{\Gamma} \mathbf{U}+\mu_{\mathrm{int}}\left[\nabla_{\Gamma} \mathbf{U}\right] \mathbf{n} \\
& \mathbf{B}_{t}^{1} \mathbf{U}:=-\left(\lambda_{\mathrm{int}} \mathbf{n}\left(\operatorname{Tr}\left(\left[\mathrm{D}^{2} b\right] \nabla_{\Gamma} \mathbf{U}\right)\right)+\mu_{\mathrm{int}}\left(\left[\mathrm{D}^{2} b\right] \nabla_{\Gamma} \mathbf{U}\right) \mathbf{n}\right) .
\end{aligned}
$$

Secondly we set $\boldsymbol{u}_{e}^{\varepsilon}(x):=\sum_{n \geq 0} \varepsilon^{n} \boldsymbol{u}_{e}^{n}(x)$ in $\Omega \backslash \bar{\omega}$ and $\boldsymbol{u}_{i}^{\varepsilon}(x, s):=\mathbf{U}_{i}^{\varepsilon}(x, S)=\sum_{n \geq 0} \varepsilon^{n} \mathbf{U}_{i}^{n}(x, S)$ in $\Gamma \times[0,1]$. Hence the transmission problem $[1.2$ can be rewritten as follows:

$$
\left\{\begin{aligned}
-\sum_{n \geq 0} \varepsilon^{n} \operatorname{div} A_{\mathrm{ext}} e\left(\boldsymbol{u}_{e}^{n}\right) & =\boldsymbol{f} & & \text { in } \Omega \backslash \bar{\omega} \\
\sum_{n \geq 0} \varepsilon^{n} \partial_{S}^{2} \Lambda_{0} \mathbf{U}_{i}^{n} & =-\sum_{n \geq 1} \varepsilon^{n} \Lambda_{1} \partial_{S} \mathbf{U}_{i}^{n-1}-\sum_{n \geq 2} \varepsilon^{n} \Lambda_{2} \mathbf{U}_{i}^{n-2}-\cdots & & \text { in } \Gamma \times(0,1) \\
\sum_{n \geq 0} \varepsilon^{n} \boldsymbol{u}_{e}^{n} & =\mathbf{0} & & \text { on } \Gamma_{D} \\
\sum_{n \geq 0} \varepsilon^{n}\left(A_{\mathrm{ext}} e\left(\boldsymbol{u}_{e}^{n}\right)\right) \mathbf{n} & =\boldsymbol{g} & & \text { on } \Gamma_{N} \\
\sum_{n \geq 1}^{n} \varepsilon^{n}\left(A_{\mathrm{ext}} e\left(\boldsymbol{u}_{e}^{n-1}\right)\right) \mathbf{n} & =\sum_{n \geq 0} \varepsilon^{n} \partial_{S} \Lambda_{0} \mathbf{U}_{i}^{n}+\sum_{n \geq 1} \varepsilon^{n} \mathbf{B}_{t}^{0} \mathbf{U}_{i}^{n-1} & & \text { on } \Gamma \times\{0\} \\
\sum_{n \geq 0} \varepsilon^{n} \Lambda_{0} \boldsymbol{u}_{e}^{n} & =\sum_{n \geq 0}^{n} \varepsilon^{n} \Lambda_{0} \mathbf{U}_{i}^{n} & & \text { on } \Gamma \times\{0\} \\
\sum_{n \geq 0} \varepsilon^{n} \partial_{S} \Lambda_{0} \mathbf{U}_{i}^{n} & =-\sum_{n \geq 1} \varepsilon^{n} \mathbf{B}_{t}^{0} \mathbf{U}_{i}^{n-1}-\sum_{n \geq 2} \varepsilon^{n} \mathbf{B}_{t}^{1} \mathbf{U}_{i}^{n-2}+\cdots & & \text { on } \Gamma \times\{1\} .
\end{aligned}\right.
$$

Then we prove the following result.

Proposition 2.1. The $G I B C$, defined on $\Gamma$, modeling the interior thin layer effects of the inclusion $\omega$ for $N=0,1$, are given by

$$
C\left(\varepsilon, A_{\mathrm{ext}} e\left(\boldsymbol{v}_{[N]}^{\varepsilon}\right) \mathbf{n}, \boldsymbol{v}_{[N]}^{\varepsilon}\right):=\left(A_{\mathrm{ext}} e\left(\boldsymbol{v}_{[N]}^{\varepsilon}\right) \mathbf{n}\right)+\mathbf{C}^{\varepsilon, N} \boldsymbol{v}_{[N]}^{\varepsilon},
$$

where $\mathbf{C}^{\varepsilon, 0}:=\mathbf{0}$ and $\mathbf{C}^{\varepsilon, 1} \boldsymbol{v}:=-\varepsilon \operatorname{div}_{\Gamma}\left(\bar{A}_{\text {int }} e_{\Gamma}(\boldsymbol{v})\right)$.

Proof. The rank $n=0$ allows us to compute $\mathbf{U}_{i}^{0}$ only. The solution $\mathbf{U}_{i}^{0}$ is characterized by

$$
\left\{\begin{aligned}
\partial_{S}^{2} \Lambda_{0} \mathbf{U}_{i}^{0} & =\mathbf{0} & & \text { in } \Gamma \times(0,1) \\
\partial_{S} \Lambda_{0} \mathbf{U}_{i}^{0} & =\mathbf{0} & & \text { on } \Gamma \times\{1\} \\
\Lambda_{0} \mathbf{U}_{i}^{0} & =\Lambda_{0} \boldsymbol{u}_{e}^{0} & & \text { on } \Gamma \times\{0\}
\end{aligned}\right.
$$

We deduce $\Lambda_{0} \mathbf{U}_{i}^{0}(x, S)=\Lambda_{0} \boldsymbol{u}_{e \mid \Gamma}^{0}(x)$.

When $n=1$, we obtain the two systems

$$
\left\{\begin{aligned}
\partial_{S}^{2} \Lambda_{0} \mathbf{U}_{i}^{1} & =-\Lambda_{1} \partial_{S} \mathbf{U}_{i}^{0}=\mathbf{0} & & \text { in } \Gamma \times(0,1) \\
\partial_{S} \Lambda_{0} \mathbf{U}_{i}^{1} & =-\mathbf{B}_{t}^{0} \mathbf{U}_{i}^{0}=-\mathbf{B}_{t}^{0} \boldsymbol{u}_{e \mid \Gamma}^{0} & & \text { on } \Gamma \times\{1\} \\
\Lambda_{0} \mathbf{U}_{i}^{1} & =\Lambda_{0} \boldsymbol{u}_{e}^{1} & & \text { on } \Gamma \times\{0\}
\end{aligned}\right.
$$

and

$$
\left\{\begin{aligned}
-\operatorname{div} A_{\mathrm{ext}} e\left(\boldsymbol{u}_{e}^{0}\right) & =\boldsymbol{f} & & \text { in } \Omega \backslash \bar{\omega} \\
\boldsymbol{u}_{e}^{0} & =\mathbf{0} & & \text { on } \Gamma_{D} \\
\left(A_{\mathrm{ext}} e\left(\boldsymbol{u}_{e}^{0}\right)\right) \mathbf{n} & =\boldsymbol{g} & & \text { on } \Gamma_{N} \\
\left(A_{\mathrm{ext}} e\left(\boldsymbol{u}_{e}^{0}\right)\right) \mathbf{n} & =\partial_{S} \Lambda_{0} \mathbf{U}_{i}^{1}+\mathbf{B}_{t}^{0} \mathbf{U}_{i}^{0} & & \text { on } \Gamma .
\end{aligned}\right.
$$


We obtain $\Lambda_{0} \mathbf{U}_{i}^{1}(x, S)=-S \mathbf{B}_{t}^{0} \boldsymbol{u}_{e \mid \Gamma}^{0}(x)+\Lambda_{0} \boldsymbol{u}_{e \mid \Gamma}^{1}(x)$ and

$$
\left(A_{\text {ext }} e\left(\boldsymbol{u}_{e}^{0}\right)\right) \mathbf{n}=\partial_{S} \Lambda_{0} \mathbf{U}_{i}^{1}(\cdot, 0)+\mathbf{B}_{t}^{0} \mathbf{U}_{i}^{0}(\cdot, 0)=\mathbf{0} .
$$

In this case we approximate $\boldsymbol{u}_{e}^{\varepsilon}$ by the function $\boldsymbol{v}_{[0]}^{\varepsilon}=\boldsymbol{u}_{e}^{0}$ and then $\left(A_{\text {ext }} e\left(\boldsymbol{u}_{e}^{\varepsilon}-\boldsymbol{v}_{[0]}^{\varepsilon}\right)\right) \mathbf{n}=\mathbf{0}=O(\varepsilon)$.

When $n=2$, we obtain the two systems

$$
\left\{\begin{aligned}
\partial_{S}^{2} \Lambda_{0} \mathbf{U}_{i}^{2} & =-\Lambda_{1} \partial_{S} \mathbf{U}_{i}^{1}-\Lambda_{2,2} \mathbf{U}_{i}^{0} & & \text { in } \Gamma \times(0,1) \\
\partial_{S} \Lambda_{0} \mathbf{U}_{i}^{2} & =-\mathbf{B}_{t}^{0} \mathbf{U}_{i}^{1}-\mathbf{B}_{t}^{1} \mathbf{U}_{i}^{0} & & \text { on } \Gamma \times\{1\} \\
\Lambda_{0} \mathbf{U}_{i}^{2} & =\Lambda_{0} \boldsymbol{u}_{e}^{2} & & \text { on } \Gamma \times\{0\}
\end{aligned}\right.
$$

and

$$
\left\{\begin{aligned}
-\operatorname{div} A_{\text {ext }} e\left(\boldsymbol{u}_{e}^{1}\right) & =\mathbf{0} & & \text { un } \Omega \backslash \bar{\omega} \\
\boldsymbol{u}_{e}^{1} & =\mathbf{0} & & \text { on } \Gamma_{D} \\
\left(A_{\text {ext }} e\left(\boldsymbol{u}_{e}^{1}\right)\right) \mathbf{n} & =\mathbf{0} & & \text { on } \Gamma_{N} \\
\left(A_{\text {ext }} e\left(\boldsymbol{u}_{e}^{1}\right)\right) \mathbf{n} & =\partial_{S} \Lambda_{0} \mathbf{U}_{i}^{2}+\mathbf{B}_{t}^{0} \mathbf{U}_{i}^{1} & & \text { on } \Gamma .
\end{aligned}\right.
$$

We compute $\partial_{S}^{2} \Lambda_{0} \mathbf{U}_{i}^{2}(x, S)=\Lambda_{1} \Lambda_{0}^{-1} \mathbf{B}_{t}^{0} \boldsymbol{u}_{e \mid \Gamma}^{0}(x)-\Lambda_{2,2} \boldsymbol{u}_{e \mid \Gamma}^{0}(x)$ and we obtain

$$
\begin{aligned}
\Lambda_{0} \mathbf{U}_{i}^{2}(\cdot, S)=\left(\frac{S^{2}}{2}-S\right)\left(\Lambda_{1} \Lambda_{0}^{-1} \mathbf{B}_{t}^{0}-\Lambda_{2,2}\right) & \boldsymbol{u}_{e \mid \Gamma}^{0} \\
+ & S\left(\mathbf{B}_{t}^{0} \Lambda_{0}^{-1} \mathbf{B}_{t}^{0} \boldsymbol{u}_{e \mid \Gamma}^{0}-\mathbf{B}_{t}^{0} \boldsymbol{u}_{e \mid \Gamma}^{1}-\mathbf{B}_{t}^{1} \boldsymbol{u}_{e \mid \Gamma}^{0}\right)+\Lambda_{0} \boldsymbol{u}_{e \mid \Gamma}^{2}
\end{aligned}
$$

and

$$
\left(A_{\mathrm{ext}} e\left(\boldsymbol{u}_{e}^{1}\right)\right) \mathbf{n}=\partial_{S} \Lambda_{0} \mathbf{U}_{i}^{2}(\cdot, 0)+\mathbf{B}_{t}^{0} \mathbf{U}_{i}^{1}(\cdot, 0)=-\left(\Lambda_{1} \Lambda_{0}^{-1} \mathbf{B}_{t}^{0}-\Lambda_{2,2}-\mathbf{B}_{t}^{0} \Lambda_{0}^{-1} \mathbf{B}_{t}^{0}+\mathbf{B}_{t}^{1}\right) \boldsymbol{u}_{i \mid \Gamma}^{0} .
$$

We find

$$
\begin{aligned}
& \mathbf{B}_{t}^{0} \Lambda_{0}^{-1} \mathbf{B}_{t}^{0}=\left(\lambda_{\mathrm{int}} \mathbf{n} \operatorname{div}_{\Gamma} \cdot+\mu_{\mathrm{int}}\left[\nabla_{\Gamma} \cdot\right] \mathbf{n}\right)\left(\frac{\lambda_{\mathrm{int}}}{\lambda_{\mathrm{int}}+2 \mu_{\mathrm{int}}} \mathbf{n d i v}_{\Gamma} \cdot+\left[\nabla_{\Gamma} \cdot\right] \mathbf{n}\right) \\
& =\frac{\lambda_{\text {int }}^{2}}{\lambda_{\text {int }}+2 \mu_{\text {int }}} \operatorname{Hn} \operatorname{div}_{\Gamma}+\lambda_{\text {int }} \mathbf{n}\left(\mathbf{n} \cdot \Delta_{\Gamma}\right)+\lambda_{\text {int }} \mathbf{n} \operatorname{Tr}\left(\left[\mathrm{D}^{2} b\right] \nabla_{\Gamma} \cdot\right) \\
& +\frac{\lambda_{\text {int }} \mu_{\text {int }}}{\lambda_{\text {int }}+2 \mu_{\text {int }}} \nabla_{\Gamma} \operatorname{div}_{\Gamma}-\mu_{\text {int }}\left(\left[D^{2} b\right] \nabla_{\Gamma^{*}}\right) \mathbf{n}, \\
& \Lambda_{1} \Lambda_{0}^{-1} \mathbf{B}_{t}^{0}=\Lambda_{1}\left(\frac{\lambda_{\text {int }}}{\lambda_{\text {int }}+2 \mu_{\text {int }}} \mathbf{n} \operatorname{div}_{\Gamma} \cdot+\left[\nabla_{\Gamma} \cdot\right] \mathbf{n}\right) \\
& =\lambda_{\text {int }} \mathbf{n H d i v}{ }_{\Gamma}+\mu_{\text {int }} \mathrm{H}\left[\nabla_{\Gamma} \cdot \mathbf{n}+\left(\lambda_{\text {int }}+\mu_{\text {int }}\right) \mathbf{n}\left(\mathbf{n} \cdot \Delta_{\Gamma}\right)\right. \\
& +\frac{\lambda_{\text {int }}\left(\lambda_{\text {int }}+\mu_{\text {int }}\right)}{\lambda_{\text {int }}+2 \mu_{\text {int }}} \nabla_{\Gamma} \operatorname{div}_{\Gamma}+\left(\lambda_{\text {int }}+\mu_{\text {int }}\right) n \operatorname{Tr}\left(\left[\mathrm{D}^{2} b\right] \nabla_{\Gamma^{*}}\right), \\
& \Lambda_{1} \Lambda_{0}^{-1} \mathbf{B}_{t}^{0}-\mathbf{B}_{t}^{0} \Lambda_{0}^{-1} \mathbf{B}_{t}^{0}+\mathbf{B}_{t}^{1}=\lambda_{\operatorname{int}} \mathbf{n H d i v}{ }_{\Gamma}+\mu_{\mathrm{int}} \mathrm{H}\left[\nabla_{\Gamma} \cdot\right] \mathbf{n}+\mu_{\mathrm{int}} \mathbf{n}\left(\mathbf{n} \cdot \Delta_{\Gamma}\right) \\
& +\frac{\lambda_{\text {int }}^{2}}{\lambda_{\text {int }}+2 \mu_{\text {int }}}\left(\nabla_{\Gamma} \operatorname{div}_{\Gamma}-\operatorname{Hn} \operatorname{div}_{\Gamma}\right)-\left(\lambda_{\text {int }}-\mu_{\text {int }}\right) \mathbf{n} \operatorname{Tr}\left(\left[\mathrm{D}^{2} b\right] \nabla_{\Gamma^{*}}\right) .
\end{aligned}
$$

We finally use

$$
\begin{aligned}
\nabla_{\Gamma} \operatorname{div}_{\Gamma}-\operatorname{Hndiv}_{\Gamma} & =\operatorname{div}_{\Gamma}\left(\left(\operatorname{div}_{\Gamma}\right)\left(\mathrm{I}_{d}-\mathbf{n} \otimes \mathbf{n}\right)\right), \\
\mathrm{H}\left[\nabla_{\Gamma^{*}}\right] \mathbf{n}+\mathbf{n}\left(\mathbf{n} \cdot \Delta_{\Gamma}\right)+\mathbf{n} \operatorname{Tr}\left(\left[\mathrm{D}^{2} b\right] \nabla_{\Gamma^{*}}\right)+\left(\left[\mathrm{D}^{2} b\right] \nabla_{\Gamma}\right) \mathbf{n} & =\operatorname{div}_{\Gamma}\left(\mathbf{n} \otimes\left[\nabla_{\Gamma^{*}}\right] \mathbf{n}+\left[\nabla_{\Gamma^{*}}\right] \mathbf{n} \otimes \mathbf{n}\right)
\end{aligned}
$$
to get

$$
\left(A_{\mathrm{ext}} e\left(\boldsymbol{u}_{e}^{1}\right)\right) \mathbf{n}=\operatorname{div}_{\Gamma}\left(\frac{2 \lambda_{\mathrm{int}} \mu_{\mathrm{int}}}{\lambda_{\mathrm{int}}+2 \mu_{\mathrm{int}}}\left(\operatorname{div}_{\Gamma} \boldsymbol{u}_{e}^{0}\right) \Pi_{d}+\mu_{\mathrm{int}} \Pi_{d}\left(\left[\nabla_{\Gamma} \boldsymbol{u}_{e}^{0}\right]+{ }^{t}\left[\nabla_{\Gamma} \boldsymbol{u}_{e}^{0}\right]\right) \Pi_{d}\right) .
$$

In this case we approximate the solution $\boldsymbol{u}_{e}^{\varepsilon}$ by the function $\boldsymbol{v}_{[1]}^{\varepsilon}$ that satisfies Problem 2.1] with the following boundary condition on $\Gamma$

$$
\left(A_{\mathrm{ext}} e\left(\boldsymbol{v}_{[1]}^{\varepsilon}\right)\right) \mathbf{n}-\varepsilon \operatorname{div}_{\Gamma}\left(\frac{2 \lambda_{\mathrm{int}} \mu_{\mathrm{int}}}{\lambda_{\mathrm{int}}+2 \mu_{\mathrm{int}}}\left(\operatorname{div}_{\Gamma} \boldsymbol{v}_{[1]}^{\varepsilon}\right) \Pi_{d}+\mu_{\mathrm{int}} \Pi_{d}\left(\left[\nabla_{\Gamma} \boldsymbol{v}_{[1]}^{\varepsilon}\right]+{ }^{t}\left[\nabla_{\Gamma} \boldsymbol{v}_{[1]}^{\varepsilon}\right]\right) \Pi_{d}\right)=\mathbf{0} .
$$


below.

Setting $\boldsymbol{u}_{[1]}^{\varepsilon}:=\boldsymbol{u}_{e}^{0}+\varepsilon \boldsymbol{u}_{e}^{1}$, we deduce the following estimate which permits to prove Theorem 2.3

Lemma 2.2. We have

$$
\begin{aligned}
\left(A _ { \mathrm { ext } } e \left(\boldsymbol{u}_{[1]}^{\varepsilon}\right.\right. & \left.\left.-\boldsymbol{v}_{[1]}^{\varepsilon}\right)\right) \mathbf{n}+\mathbf{C}^{\varepsilon, 1}\left(\boldsymbol{u}_{[1]}^{\varepsilon}-\boldsymbol{v}_{[1]}^{\varepsilon}\right) \\
& =\varepsilon^{2} \operatorname{div}_{\Gamma}\left(\frac{2 \lambda_{\mathrm{int}} \mu_{\mathrm{int}}}{\lambda_{\mathrm{int}}+2 \mu_{\mathrm{int}}}\left(\operatorname{div}_{\Gamma} \boldsymbol{u}_{e}^{1}\right) \Pi_{d}+\mu_{\mathrm{int}} \Pi_{d}\left(\left[\nabla_{\Gamma} \boldsymbol{u}_{e}^{1}\right]+{ }^{t}\left[\nabla_{\Gamma} \boldsymbol{u}_{e}^{1}\right]\right) \Pi_{d}\right)=O\left(\varepsilon^{2}\right) .
\end{aligned}
$$

\subsection{Convergence analysis (Proof of Theorem 1.2)}

We focus now on the remainder $\boldsymbol{r}_{[N]}^{\varepsilon}:=\boldsymbol{v}_{[N]}^{\varepsilon}-\boldsymbol{u}_{e}^{\varepsilon}$ for $N=0,1$. We obtain the following result which proves Theorem 1.2

Theorem 2.3. Let $N=0,1$. If $\boldsymbol{f} \in \mathbf{H}^{1 / 2}(\Omega \backslash \bar{\omega})$, then there exists a constant $C_{\Omega \backslash \bar{\omega}}$ independent on $\varepsilon$ such that

$$
\left\|\boldsymbol{r}_{[N]}^{\varepsilon}\right\|_{\mathbf{H}^{1}(\Omega \backslash \bar{\omega})} \leq C_{\Omega \backslash \bar{\omega}} \varepsilon^{N+1} .
$$

Proof. We follow the procedure described in the proof of [23, Theorem 4.1]. The idea is to decompose the remainder, for $N=0,1$, as $\boldsymbol{r}_{[N]}^{\varepsilon}:=\boldsymbol{v}_{[N]}^{\varepsilon}-\boldsymbol{u}_{[N]}^{\varepsilon}+\boldsymbol{u}_{[N]}^{\varepsilon}-\boldsymbol{u}_{e}^{\varepsilon}$, where $\boldsymbol{u}_{[0]}^{\varepsilon}:=\boldsymbol{u}_{e}^{0}$ and $\boldsymbol{u}_{[1]}^{\varepsilon}:=\boldsymbol{u}_{e}^{0}+\varepsilon \boldsymbol{u}_{e}^{1}$.

Firstly we set $\boldsymbol{R}_{\varepsilon}^{N}:=\boldsymbol{u}^{\varepsilon}-\sum_{n=0}^{N} \varepsilon^{n} \boldsymbol{u}^{n}$ and we denote by $\boldsymbol{R}_{\varepsilon, e}^{N}$ and $\boldsymbol{R}_{\varepsilon, i}^{N}$ the restriction of $\boldsymbol{R}_{\varepsilon}^{N}$ respectively to $\Omega \backslash \bar{\omega}$ and to $\omega_{\text {ext }}^{\epsilon}$. It is straightforward to show that $\boldsymbol{R}_{\varepsilon}^{N}=\left(\boldsymbol{R}_{\varepsilon, e}^{N}, \boldsymbol{R}_{\varepsilon, i}^{N}\right)$ is a solution of the following system

$$
\left\{\begin{aligned}
-\operatorname{div} A_{\text {ext }} e\left(\boldsymbol{R}_{\varepsilon, e}^{N}\right) & =\mathbf{0} & & \text { in } \Omega \backslash \bar{\omega} \\
-\operatorname{div} A_{\text {int }} e\left(\boldsymbol{R}_{\varepsilon, i}^{N}\right) & =O\left(\varepsilon^{N-1}\right) & & \text { in } \omega_{\text {int }}^{\varepsilon} \\
\boldsymbol{R}_{\epsilon, e}^{N} & =\mathbf{0} & & \text { on } \Gamma_{D} \\
A_{\text {ext }} e\left(\boldsymbol{R}_{\varepsilon, e}^{N}\right) & =\mathbf{0} & & \text { on } \Gamma_{N} \\
A_{\text {ext }} e\left(\boldsymbol{R}_{\varepsilon, e}^{N}\right) \mathbf{n} & =A_{\text {int }} e\left(\boldsymbol{R}_{\varepsilon, i}^{N}\right) \mathbf{n}+O\left(\varepsilon^{N}\right) & & \text { on } \Gamma \\
\Lambda_{0} \boldsymbol{R}_{\varepsilon, e}^{N} & =\Lambda_{0} \boldsymbol{R}_{\varepsilon, i}^{N} & & \text { on } \Gamma \\
A_{\text {int }} e\left(\boldsymbol{R}_{\varepsilon, i}^{N}\right) \mathbf{n} & =O\left(\varepsilon^{N}\right) & & \text { on } \Gamma^{\varepsilon} .
\end{aligned}\right.
$$

The remainders are considered as distributions in $\mathbf{H}^{-1}\left(\omega_{\text {int }}^{\varepsilon}\right), \mathbf{H}^{-1 / 2}(\Gamma)$ and $\mathbf{H}^{-1 / 2}\left(\Gamma^{\varepsilon}\right)$ respectively. Taking the variational form for the transmission problem, one can prove the uniform coercivity with respect to $\varepsilon$ of the associated bilinear form and the continuity of the bilinear and linear forms. We get, thanks to the Lax-Milgram theorem and the Korn's inequality, that there exists a constant $C$, depending on $N$ but independent on $\varepsilon$, such that

$$
\left\|\boldsymbol{R}_{\varepsilon, e}^{N}\right\|_{\mathbf{H}^{1}(\Omega \backslash \bar{\omega})} \leq C \varepsilon^{N-1} .
$$

To improve the precision of the remainder, we write

$$
\boldsymbol{R}_{\varepsilon, e}^{N}=\boldsymbol{R}_{\varepsilon, e}^{N+2}+\varepsilon^{N+1} \boldsymbol{u}_{e}^{N+1}+\varepsilon^{N+2} \boldsymbol{u}_{e}^{N+2}
$$

and get the estimation

$$
\left\|\boldsymbol{u}_{e}^{\varepsilon}-\boldsymbol{u}_{[N]}^{\varepsilon}\right\|_{\mathbf{H}^{1}(\Omega \backslash \bar{\omega})}=\left\|\boldsymbol{R}_{\varepsilon, e}^{N}\right\|_{\mathbf{H}^{1}(\Omega \backslash \bar{\omega})} \leq C \varepsilon^{N+1} .
$$

Secondly let us focus on $\boldsymbol{d}_{[N]}:=\boldsymbol{u}_{[N]}^{\varepsilon}-\boldsymbol{v}_{[N]}^{\varepsilon}$. For $N=0$, we have $\boldsymbol{u}_{[0]}^{\varepsilon}=\boldsymbol{u}_{e}^{0}=\boldsymbol{v}_{[0]}^{\varepsilon}$, i.e. $\boldsymbol{d}_{[0]}=\mathbf{0}$. Hence, thanks to the previous estimate 2.8), there exists a positive constant $C_{0}$, independent of $\varepsilon$, such that

$$
\left\|\boldsymbol{r}_{[0]}^{\varepsilon}\right\|_{\mathbf{H}^{1}(\Omega \backslash \bar{\omega})} \leq C_{0} \varepsilon
$$


For $N=1, \boldsymbol{d}_{[1]}=\boldsymbol{u}_{[1]}^{\varepsilon}-\boldsymbol{v}_{[1]}^{\varepsilon}$ satisfies the following system

$$
\left\{\begin{aligned}
-\operatorname{div} A_{\mathrm{ext}} e\left(\boldsymbol{d}_{[1]}\right) & =\mathbf{0} & & \text { in } \Omega \backslash \bar{\omega} \\
\boldsymbol{d}_{[1]} & =\mathbf{0} & & \text { on } \Gamma_{D} \\
A_{\mathrm{ext}} e\left(\boldsymbol{d}_{[1]}\right) \mathbf{n} & =\mathbf{0} & & \text { on } \Gamma_{N} \\
\left(A_{\mathrm{ext}} e\left(\boldsymbol{d}_{[1]}\right) \mathbf{n}+\mathbf{C}^{\varepsilon, 1}\left(\boldsymbol{d}_{[1]}\right)\right. & =\varepsilon^{2} \operatorname{div}_{\Gamma}\left(\sigma_{\Gamma}\left(\boldsymbol{u}_{e}^{1}\right)\right) & & \text { on } \Gamma .
\end{aligned}\right.
$$

Let us notice that the last equality comes from Lemma 2.2 and the right hand side is considered as a distribution in $\mathbf{V}^{\prime}(\Gamma)$. Writing the variational form, we deduce, thanks to Proposition A.1 and Remark 1.1, that there exist two positive constants $C, C^{\prime}>0$ such that

$$
\left\|\boldsymbol{d}_{[1]}\right\|_{\mathcal{H}(\Omega \backslash \bar{\omega})} \leq C \varepsilon^{2}\left\|\operatorname{div}_{\Gamma}\left(\sigma_{\Gamma}\left(\boldsymbol{u}_{e}^{1}\right)\right)\right\|_{\mathbf{V}^{\prime}(\Gamma)} \leq C^{\prime} \varepsilon^{2}\left\|\boldsymbol{u}_{e}^{1}\right\|_{\mathbf{v}(\Gamma)}
$$

Moreover, thanks to the condition $\boldsymbol{f} \in \mathbf{H}^{1 / 2}(\Omega \backslash \bar{\omega})$, the local regularity in a neighborhood of $\omega$ ensures that the solution $\boldsymbol{u}_{e}^{0}$ of Problem (2.3) (with the boundary condition given by 2.4)) belongs to $\mathbf{H}^{5 / 2}\left(\Omega_{\delta} \backslash \bar{\omega}\right)$ for some smooth open set $\Omega_{\delta}$ such that $\omega \subset \subset \Omega_{\delta} \subset \subset \Omega \backslash \bar{\omega}$. Then $\left.\boldsymbol{u}_{e}^{0}\right|_{\Gamma} \in \mathbf{H}^{2}(\Gamma)$ and $\left.\operatorname{div}_{\Gamma}\left(\sigma_{\Gamma}\left(\boldsymbol{u}_{e}^{0}\right)\right)\right|_{\Gamma} \in \mathbf{L}^{2}(\Gamma)$. Using the local regularity of the solution $\boldsymbol{u}_{e}^{1}$ of Problem 2.5) with the Neumann boundary condition $(2.6)$, we obtain that there exists $C^{\prime \prime}>0$ such that

$$
\left\|\boldsymbol{u}_{e}^{1}\right\|_{\mathbf{H}^{3 / 2}\left(\Omega_{\delta} \backslash \bar{\omega}\right)} \leq C^{\prime \prime}\left\|\operatorname{div}_{\Gamma}\left(\sigma_{\Gamma}\left(\boldsymbol{u}_{e}^{0}\right)\right)\right\|_{\mathbf{L}^{2}(\Gamma)} .
$$

Finally, using the continuity of the trace operator and the continuous embedding $\mathbf{H}^{1}(\Gamma) \subset \mathbf{V}(\Gamma)$, we obtain that there exists $C^{\prime \prime \prime}>0$

$$
\left\|\boldsymbol{d}_{[1]}\right\|_{\mathcal{H}(\Omega \backslash \bar{\omega})} \leq C^{\prime \prime \prime} \varepsilon^{2}\left\|\operatorname{div}_{\Gamma}\left(\sigma_{\Gamma}\left(\boldsymbol{u}_{e}^{0}\right)\right)\right\|_{\mathbf{L}^{2}(\Gamma)} .
$$

Using the triangular inequality and inequality (2.8), we conclude that there exists a positive constant $C_{1}$, independent of $\varepsilon$, such that

$$
\left\|\boldsymbol{r}_{[1]}^{\varepsilon}\right\|_{\mathbf{H}^{1}(\Omega \backslash \bar{\omega})} \leq C_{1} \varepsilon^{2}
$$

Remark 2.4. The regularity results used in the above proof can be found for example in 143 , Chapter 4] or in [13, Theorems 9.25 and 9.26, and Remark 24] (notice that the mentioned results in [13] concern global regularity results but the local regularity results are then classically deduced using a cut-off function).

\section{Shape sensitivity analysis}

This section is devoted to the proof of the existence of the shape derivative of the state $\boldsymbol{u}$, solution of Problem (1.4). This step is done following the general method exposed for example in [35, Chapter 5] which consists in using a change of variable and the implicit function theorem. Then we prove the characterization of the shape derivative $\boldsymbol{u}^{\prime}$ claimed in Theorem 1.3 . We conclude this section with an asymptotic analysis for the shape derivatives of the transmission problem (1.2) which shows that we can interchange the asymptotic and shape sensitivity analysis.

We have first to introduce some notations. Let us define $\Omega_{\delta}$ an open set with a $C^{\infty}$ boundary such that

$$
\{x \in \Omega ; \mathrm{d}(x, \partial \Omega)>\delta / 2\} \subset \Omega_{\delta} \subset\{x \in \Omega ; \mathrm{d}(x, \partial \Omega)>\delta / 3\} .
$$

Then, in order to make a shape sensitivity analysis, we define

$$
\mathcal{U}:=\left\{\boldsymbol{\theta} \in \boldsymbol{C}^{2, \infty}=\boldsymbol{C}^{2} \cap \mathbf{W}^{2, \infty}\left(\mathbb{R}^{d}\right) ; \operatorname{supp}(\boldsymbol{\theta}) \subset \overline{\Omega_{\delta}} \text { and }\|\boldsymbol{\theta}\|_{2, \infty}<\min \left(\frac{\delta}{3}, 1\right)\right\}
$$

as the space of admissible deformations. This space enables us to perturb only the object $\omega$ and not the fixed domain $\Omega$. 


\subsection{Existence of the shape derivatives for the GIBC model}

In this section, we prove that the solution $\boldsymbol{u} \in \mathcal{H}(\Omega \backslash \bar{\omega})$ of the GIBC Problem $(1.4)$ is differentiable with respect to the shape $\omega$. Notice that if $\boldsymbol{\theta} \in \mathcal{U}$, then $(\mathbf{I}+\boldsymbol{\theta})$ is a diffeomorphism. For such a $\boldsymbol{\theta} \in \mathcal{U}$ and $\omega \in \mathcal{O}_{\delta}$, we check that $\Omega=(\mathbf{I}+\boldsymbol{\theta})(\Omega)$ and we define the perturbed domain

$$
\omega_{\theta}:=(\mathbf{I}+\boldsymbol{\theta})(\omega) \in \mathcal{O}_{\delta} .
$$

First we need to introduce the following Piola transform and prove the following result.

Proposition 3.1. Let $\boldsymbol{\theta} \in \mathcal{U}$. The adjoint Piola transform of $(\mathbf{I}+\boldsymbol{\theta})^{-1}$ defined by

$$
\begin{aligned}
\mathcal{P}^{*}(\boldsymbol{\theta}): \mathcal{H}(\Omega \backslash \bar{\omega}) & \longrightarrow \mathcal{H}\left(\Omega \backslash \overline{\omega_{\theta}}\right) \\
\boldsymbol{u} & \longmapsto\left(\left(\mathrm{I}_{d}+\nabla \boldsymbol{\theta}\right)^{-1} \boldsymbol{u}\right) \circ(\mathbf{I}+\boldsymbol{\theta})^{-1}
\end{aligned}
$$

is an isomorphism and we denote by $\mathcal{P}^{-*}(\boldsymbol{\theta})$ its inverse.

Proof. The only point is to prove that $\mathcal{P}^{*}(\boldsymbol{\theta}) \boldsymbol{\psi}$ belongs to $\mathcal{H}\left(\Omega \backslash \overline{\omega_{\theta}}\right)$ for all $\boldsymbol{\psi} \in \mathcal{H}(\Omega \backslash \bar{\omega})$. Firstly, for $\boldsymbol{\psi} \in \mathcal{H}(\Omega \backslash \bar{\omega})$, it is obvious that $\mathcal{P}^{*}(\boldsymbol{\theta}) \boldsymbol{\psi}$ belongs to $\mathbf{H}^{1}\left(\Omega \backslash \bar{\omega}_{\theta}\right)$ and that $\boldsymbol{\psi}=\mathbf{0}$ on $\Gamma_{D}$. Secondly, the restriction of $\left(\mathrm{I}_{d}+\nabla \boldsymbol{\theta}\right)^{-1}$ to the boundary $\Gamma$ maps $\left\{\boldsymbol{\psi} \in \mathbf{H}^{1}(\Gamma) ; \boldsymbol{\psi} \cdot \mathbf{n}=\mathbf{0}\right\}$ into $\mathbf{H}^{1}(\Gamma)$. Moreover, we have [35, Proposition 5.4.14]

$$
\mathbf{n}_{\theta}:=\frac{\mathcal{P}^{*}(\boldsymbol{\theta}) \mathbf{n}}{\left\|\mathcal{P}^{*}(\boldsymbol{\theta}) \mathbf{n}\right\|} .
$$

Then $\mathcal{P}^{*}(\boldsymbol{\theta})$ is an isomorphism from $\mathbf{V}(\Gamma)$ to $\mathbf{V}\left(\Gamma_{\theta}\right)$ (i.e. the tangential component remains in $\left.\left\{\boldsymbol{\psi} \in \mathbf{H}^{1}\left(\Gamma_{\theta}\right) ; \boldsymbol{\psi} \cdot \mathbf{n}_{\theta}=\mathbf{0}\right\}\right)$.

Let $\boldsymbol{\theta} \in \mathcal{U}$. In what follows, $\nabla_{\Gamma_{\theta}}$ and $\operatorname{div}_{\Gamma_{\theta}}$ are respectively the tangential gradient and surface divergence operators according to the boundary $\Gamma_{\theta}$. Let consider the solution $\boldsymbol{u}_{\theta} \in \mathcal{H}\left(\Omega \backslash \bar{\omega}_{\theta}\right)$ of the following perturbed problem

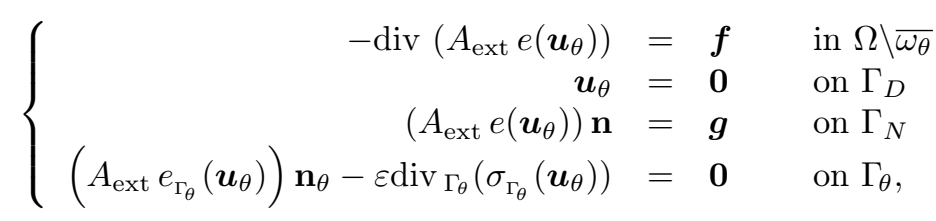

where $\mathbf{n}_{\theta}$ represents the exterior unit normal to $\Gamma_{\theta}$ given by $(3.2)$, where

$$
e_{\Gamma_{\theta}}\left(\boldsymbol{u}_{\theta}\right):=\frac{1}{2} \Pi_{d, \theta}\left(\nabla_{\Gamma_{\theta}} \boldsymbol{u}_{\theta}+{ }^{t} \nabla_{\Gamma_{\theta}} \boldsymbol{u}_{\theta}\right) \Pi_{d, \theta} \quad \text { and } \quad \Pi_{d, \theta}:=\mathrm{I}_{d}-\mathbf{n}_{\theta} \otimes \mathbf{n}_{\theta},
$$

and where

$$
\sigma_{\Gamma_{\theta}}\left(\boldsymbol{u}_{\theta}\right):=\bar{\lambda}_{\mathrm{int}}\left(\operatorname{div}_{\Gamma_{\theta}} \boldsymbol{u}_{\theta}\right) \Pi_{d, \theta}+2 \mu_{\mathrm{int}} e_{\Gamma_{\theta}}\left(\boldsymbol{u}_{\theta}\right) .
$$

Then $\boldsymbol{u}_{\theta} \in \mathcal{H}\left(\Omega \backslash \overline{\omega_{\theta}}\right)$ is the solution of

$$
\begin{aligned}
& \int_{\Omega \backslash \bar{\omega}_{\theta}} A_{\mathrm{ext}} e\left(\boldsymbol{u}_{\theta}\right): e\left(\boldsymbol{\varphi}_{\theta}\right)-\int_{\Gamma_{N}} \boldsymbol{g} \cdot \boldsymbol{\varphi}_{\theta}+\varepsilon \int_{\Gamma_{\theta}} \sigma_{\Gamma_{\theta}}\left(\boldsymbol{u}_{\theta}\right): \nabla_{\Gamma_{\theta}} \boldsymbol{\varphi}_{\theta}=\int_{\Omega \backslash \overline{\omega_{\theta}}} \boldsymbol{f} \cdot \boldsymbol{\varphi}_{\theta}, \\
& \forall \boldsymbol{\varphi}_{\theta} \in \mathcal{H}\left(\Omega \backslash \overline{\omega_{\theta}}\right) .
\end{aligned}
$$

We will apply the general method exposed in [35, Chapter 5] and [27, Section 3.1] using the adjoint Piola transform and the implicit function theorem on

$$
\boldsymbol{u}^{\theta}:=\mathcal{P}^{-*}(\boldsymbol{\theta}) \boldsymbol{u}_{\theta} \in \mathcal{H}(\Omega \backslash \bar{\omega}) .
$$


Lemma 3.2. For $\boldsymbol{\theta} \in \mathcal{U}, \boldsymbol{u}^{\theta} \in \mathcal{H}(\Omega \backslash \bar{\omega})$ satisfies for all $\boldsymbol{\varphi} \in \mathcal{H}(\Omega \backslash \bar{\omega})$

$$
\begin{aligned}
\lambda_{\text {ext }} \int_{\Omega \backslash \bar{\omega}} J_{\boldsymbol{\theta}}\left({ }^{t} \mathcal{D}_{1}(\boldsymbol{\theta}): \nabla\left(\mathcal{D}_{1}(\boldsymbol{\theta}) \boldsymbol{u}^{\theta}\right)\right)\left({ }^{t} \mathcal{D}_{1}(\boldsymbol{\theta}): \nabla\left(\mathcal{D}_{1}(\boldsymbol{\theta}) \boldsymbol{\varphi}\right)\right) \\
\quad+\mu_{\mathrm{ext}} \int_{\Omega \backslash \bar{\omega}} J_{\boldsymbol{\theta}}\left(\mathcal{D}_{1}(\boldsymbol{\theta}) \nabla\left(\mathcal{D}_{1}(\boldsymbol{\theta}) \boldsymbol{u}^{\theta}\right)\right):\left(\mathcal{D}_{1}(\boldsymbol{\theta}) \nabla\left(\mathcal{D}_{1}(\boldsymbol{\theta}) \boldsymbol{\varphi}\right)+{ }^{t}\left(\mathcal{D}_{1}(\boldsymbol{\theta}) \nabla\left(\mathcal{D}_{1}(\boldsymbol{\theta}) \boldsymbol{\varphi}\right)\right)\right) \\
\quad-\int_{\Gamma_{N}} \boldsymbol{g} \cdot \boldsymbol{\varphi}+\varepsilon \bar{\lambda}_{\mathrm{int}} \int_{\Gamma} w_{\boldsymbol{\theta}}\left({ }^{t} \mathcal{D}_{2}(\boldsymbol{\theta}): \nabla_{\Gamma}\left(\mathcal{D}_{1}(\boldsymbol{\theta}) \boldsymbol{u}^{\theta}\right)\right)\left({ }^{t} \mathcal{D}_{2}(\boldsymbol{\theta}): \nabla_{\Gamma}\left(\mathcal{D}_{1}(\boldsymbol{\theta}) \boldsymbol{\varphi}\right)\right) \\
\quad+\varepsilon \mu_{\text {int }} \int_{\Gamma} w_{\boldsymbol{\theta}}\left(\mathcal{D}_{2}(\boldsymbol{\theta}) \nabla_{\Gamma}\left(\mathcal{D}_{1}(\boldsymbol{\theta}) \boldsymbol{u}^{\theta}\right)\right):\left(\mathcal{D}_{2}(\boldsymbol{\theta}) \nabla_{\Gamma}\left(\mathcal{D}_{1}(\boldsymbol{\theta}) \boldsymbol{u}^{\theta}\right)+{ }^{t}\left(\mathcal{D}_{2}(\boldsymbol{\theta}) \nabla_{\Gamma}\left(\mathcal{D}_{1}(\boldsymbol{\theta}) \boldsymbol{u}^{\theta}\right)\right)\right) \\
-\varepsilon \mu_{\mathrm{int}} \int_{\Gamma} w_{\boldsymbol{\theta}}\left(\mathcal{D}_{2}(\boldsymbol{\theta}) \nabla_{\Gamma}\left(\mathcal{D}_{1}(\boldsymbol{\theta}) \boldsymbol{u}^{\theta}\right) \mathbf{n}^{\theta}\right) \cdot\left(\mathcal{D}_{2}(\boldsymbol{\theta}) \nabla_{\Gamma}\left(\mathcal{D}_{1}(\boldsymbol{\theta}) \boldsymbol{\varphi}\right) \mathbf{n}^{\theta}\right)=\int_{\Omega \backslash \bar{\omega}}(\boldsymbol{f} \circ(\mathbf{I}+\boldsymbol{\theta})) \cdot \mathcal{D}_{1}(\boldsymbol{\theta}) \boldsymbol{\varphi} J_{\theta},
\end{aligned}
$$

where $\mathbf{n}^{\theta}:=\mathbf{n}_{\theta} \circ(\mathbf{I}+\boldsymbol{\theta})$ and where

$$
\begin{aligned}
J_{\boldsymbol{\theta}} & :=\operatorname{det}\left(\mathrm{I}_{d}+\nabla \boldsymbol{\theta}\right) \in \mathrm{W}^{1, \infty}\left(\mathbb{R}^{d}\right), \\
w_{\theta} & :=\operatorname{det}\left(\mathrm{I}_{d}+\nabla \boldsymbol{\theta}\right)\left\|t\left(\mathrm{I}_{d}+\nabla \boldsymbol{\theta}\right)^{-1} \mathbf{n}\right\| \in \mathrm{W}^{1, \infty}\left(\mathbb{R}^{d}\right), \\
\mathcal{D}_{1}(\boldsymbol{\theta}) & :=\left(\mathrm{I}_{d}+\nabla \boldsymbol{\theta}\right)^{-1} \in C^{1}\left(\mathbb{R}^{d}, \mathcal{M}_{d, d}\right), \\
\mathcal{D}_{2}(\boldsymbol{\theta}) & :=\left(\mathrm{I}_{d}+\nabla_{\Gamma} \boldsymbol{\theta}\right)^{-1} \in C^{1}\left(\Gamma, \mathcal{M}_{d, d}\right) .
\end{aligned}
$$

Proof. Let $\boldsymbol{\varphi} \in \mathcal{H}(\Omega \backslash \bar{\omega})$. We define $\varphi_{\boldsymbol{\theta}}:=\mathcal{P}^{*}(\boldsymbol{\theta}) \boldsymbol{\varphi} \in \mathcal{H}\left(\Omega \backslash \overline{\omega_{\theta}}\right)$. Thus, using $\varphi_{\boldsymbol{\theta}}$ as a test function in the variational formulation (3.4), we obtain

$$
\begin{aligned}
\lambda_{\text {ext }} \int_{\Omega \backslash \overline{\omega_{\theta}}}\left(\operatorname{div}\left(\mathcal{P}^{*}(\boldsymbol{\theta}) \boldsymbol{u}^{\theta}\right)\right)\left(\operatorname{div}\left(\mathcal{P}^{*}(\boldsymbol{\theta}) \boldsymbol{\varphi}\right)\right)+2 \mu_{\mathrm{ext}} \int_{\Omega \backslash \overline{\omega_{\theta}}} \nabla\left(\mathcal{P}^{*}(\boldsymbol{\theta}) \boldsymbol{u}^{\theta}\right): e\left(\mathcal{P}^{*}(\boldsymbol{\theta}) \boldsymbol{\varphi}\right) \\
\quad-\int_{\Gamma_{N}} \boldsymbol{g} \cdot\left(\mathcal{P}^{*}(\boldsymbol{\theta}) \boldsymbol{\varphi}\right)+\varepsilon \bar{\lambda}_{\mathrm{int}} \int_{\Gamma_{\theta}}\left(\operatorname{div}_{\Gamma_{\theta}}\left(\mathcal{P}^{*}(\boldsymbol{\theta}) \boldsymbol{u}^{\theta}\right)\right)\left(\operatorname{div}_{\Gamma_{\theta}}\left(\mathcal{P}^{*}(\boldsymbol{\theta}) \boldsymbol{\varphi}\right)\right) \\
\quad+2 \varepsilon \mu_{\mathrm{int}} \int_{\Gamma_{\theta}} \nabla_{\Gamma_{\theta}}\left(\mathcal{P}^{*}(\boldsymbol{\theta}) \boldsymbol{u}^{\theta}\right): e_{\Gamma_{\theta}}\left(\mathcal{P}^{*}(\boldsymbol{\theta}) \boldsymbol{\varphi}\right)=\int_{\Omega \backslash \overline{\omega_{\theta}}} \boldsymbol{f} \cdot\left(\mathcal{P}^{*}(\boldsymbol{\theta}) \boldsymbol{\varphi}\right) .
\end{aligned}
$$

Hence, using the change of variables $x=(\mathbf{I}+\boldsymbol{\theta})(y)$ in the integrand and the following formulas

$$
\begin{aligned}
\nabla\left(\boldsymbol{v} \circ(\mathbf{I}+\boldsymbol{\theta})^{-1}\right) \circ(\mathbf{I}+\boldsymbol{\theta}) & =\mathcal{D}_{1}(\boldsymbol{\theta}) \nabla \boldsymbol{v} \\
\operatorname{div}\left(\boldsymbol{v} \circ(\mathbf{I}+\boldsymbol{\theta})^{-1}\right) \circ(\mathbf{I}+\boldsymbol{\theta}) & =\operatorname{Tr}\left[\nabla\left(\boldsymbol{v} \circ(\mathbf{I}+\boldsymbol{\theta})^{-1}\right)\right] \circ(\mathbf{I}+\boldsymbol{\theta})={ }^{t} \mathcal{D}_{1}(\boldsymbol{\theta}): \nabla \boldsymbol{v}, \\
\nabla_{\Gamma_{\theta}}\left(\boldsymbol{v} \circ(\mathbf{I}+\boldsymbol{\theta})^{-1}\right) \circ(\mathbf{I}+\boldsymbol{\theta}) & =\mathcal{D}_{2}(\boldsymbol{\theta}) \nabla_{\Gamma} \boldsymbol{v} \\
\operatorname{div}_{\Gamma_{\theta}}\left(\boldsymbol{v} \circ(\mathbf{I}+\boldsymbol{\theta})^{-1}\right) \circ(\mathbf{I}+\boldsymbol{\theta}) & =\operatorname{Tr}\left[\nabla_{\Gamma_{\theta}}\left(\boldsymbol{v} \circ(\mathbf{I}+\boldsymbol{\theta})^{-1}\right)\right] \circ(\mathbf{I}+\boldsymbol{\theta})={ }^{t} \mathcal{D}_{2}(\boldsymbol{\theta}): \nabla_{\Gamma} \boldsymbol{v}, \\
\nabla_{\Gamma_{\theta}}\left(\boldsymbol{u}_{\theta}\right): e_{\Gamma_{\theta}}\left(\boldsymbol{\varphi}_{\theta}\right) & =\frac{1}{2} \nabla_{\Gamma_{\theta}}\left(\boldsymbol{u}_{\theta}\right):\left(\nabla_{\Gamma_{\theta}}(\boldsymbol{\varphi})+{ }^{t} \nabla_{\Gamma_{\theta}}(\boldsymbol{\varphi})\right)-\frac{1}{2}\left(\nabla_{\Gamma_{\theta}}\left(\boldsymbol{u}_{\theta}\right) \mathbf{n}_{\theta}\right) \cdot\left(\nabla_{\Gamma_{\theta}}\left(\boldsymbol{\varphi}_{\theta}\right) \mathbf{n}_{\theta}\right), \\
\left(\mathcal{P}^{*}(\boldsymbol{\theta}) \boldsymbol{\varphi}\right)_{\mid \Gamma_{N}} & =\boldsymbol{\varphi}_{\mid \Gamma_{N}},
\end{aligned}
$$

we obtain the announced result.

Lemma 3.3. The function

$$
\boldsymbol{\theta} \in \mathcal{U} \mapsto \boldsymbol{u}^{\theta} \in \mathcal{H}(\Omega \backslash \bar{\omega})
$$

is differentiable in a neighborhood of $\mathbf{0}$. 
Proof. Let us consider the application $\mathcal{F}: \mathcal{U} \times \mathcal{H}(\Omega \backslash \bar{\omega}) \rightarrow \mathbf{L}^{2}(\Omega)$ defined for all $\boldsymbol{\varphi} \in \mathcal{H}(\Omega \backslash \bar{\omega})$ by

$$
\begin{aligned}
& \langle\mathcal{F}(\boldsymbol{\theta}, \boldsymbol{v}), \boldsymbol{\varphi}\rangle=\lambda_{\mathrm{ext}} \int_{\Omega \backslash \bar{\omega}} J_{\boldsymbol{\theta}}\left({ }^{t} \mathcal{D}_{1}(\boldsymbol{\theta}): \nabla\left(\mathcal{D}_{1}(\boldsymbol{\theta}) \boldsymbol{v}\right)\right)\left({ }^{t} \mathcal{D}_{1}(\boldsymbol{\theta}): \nabla\left(\mathcal{D}_{1}(\boldsymbol{\theta}) \boldsymbol{\varphi}\right)\right) \\
& +\mu_{\mathrm{ext}} \int_{\Omega \backslash \bar{\omega}} J_{\boldsymbol{\theta}}\left(\mathcal{D}_{1}(\boldsymbol{\theta}) \nabla\left(\mathcal{D}_{1}(\boldsymbol{\theta}) \boldsymbol{v}\right)\right):\left(\mathcal{D}_{1}(\boldsymbol{\theta}) \nabla\left(\mathcal{D}_{1}(\boldsymbol{\theta}) \boldsymbol{\varphi}\right)+{ }^{t}\left(\mathcal{D}_{1}(\boldsymbol{\theta}) \nabla\left(\mathcal{D}_{1}(\boldsymbol{\theta}) \boldsymbol{\varphi}\right)\right)\right) \\
& \quad-\int_{\Gamma_{N}} \boldsymbol{g} \cdot \boldsymbol{\varphi}+\varepsilon \bar{\lambda}_{\mathrm{int}} \int_{\Gamma} w_{\boldsymbol{\theta}}\left({ }^{t} \mathcal{D}_{2}(\boldsymbol{\theta}): \nabla_{\Gamma}\left(\mathcal{D}_{1}(\boldsymbol{\theta}) \boldsymbol{v}\right)\right)\left({ }^{t} \mathcal{D}_{2}(\boldsymbol{\theta}): \nabla_{\Gamma}\left(\mathcal{D}_{1}(\boldsymbol{\theta}) \boldsymbol{\varphi}\right)\right) \\
& +\varepsilon \mu_{\mathrm{int}} \int_{\Gamma} w_{\boldsymbol{\theta}}\left(\mathcal{D}_{2}(\boldsymbol{\theta}) \nabla_{\Gamma}\left(\mathcal{D}_{1}(\boldsymbol{\theta}) \boldsymbol{v}\right)\right):\left(\mathcal{D}_{2}(\boldsymbol{\theta}) \nabla_{\Gamma}\left(\mathcal{D}_{1}(\boldsymbol{\theta}) \boldsymbol{v}\right)+{ }^{t}\left(\mathcal{D}_{2}(\boldsymbol{\theta}) \nabla_{\Gamma}\left(\mathcal{D}_{1}(\boldsymbol{\theta}) \boldsymbol{v}\right)\right)\right) \\
& -\varepsilon \mu_{\mathrm{int}} \int_{\Gamma} w_{\boldsymbol{\theta}}\left(\mathcal{D}_{2}(\boldsymbol{\theta}) \nabla_{\Gamma}\left(\mathcal{D}_{1}(\boldsymbol{\theta}) \boldsymbol{v}\right) \mathbf{n}^{\theta}\right) \cdot\left(\mathcal{D}_{2}(\boldsymbol{\theta}) \nabla_{\Gamma}\left(\mathcal{D}_{1}(\boldsymbol{\theta}) \boldsymbol{\varphi}\right) \mathbf{n}^{\theta}\right)-\int_{\Omega \backslash \bar{\omega}}(\boldsymbol{f} \circ(\mathbf{I}+\boldsymbol{\theta})) \cdot \mathcal{D}_{1}(\boldsymbol{\theta}) \boldsymbol{\varphi} J_{\theta} .
\end{aligned}
$$

We have, for $\boldsymbol{\theta}=\mathbf{0}, \mathcal{F}\left(\mathbf{0}, \boldsymbol{u}^{0}\right)=\mathcal{F}(\mathbf{0}, \boldsymbol{u})=\mathbf{0}$. Moreover, we prove analogously to what is done in [35, Proof of Theorem 5.3.2] that the application $\mathcal{F}$ is $C^{1}$. Finally, we compute for all $\boldsymbol{w}, \hat{\boldsymbol{w}} \in \mathcal{H}(\Omega)$,

$$
\mathrm{D}_{\boldsymbol{u}} \mathcal{F}(\mathbf{0}, \boldsymbol{u})(\boldsymbol{w}) \cdot \hat{\boldsymbol{w}}=\int_{\Omega \backslash \bar{\omega}} A_{\mathrm{ext}} e(\boldsymbol{w}): e(\hat{\boldsymbol{w}})+\varepsilon \int_{\Gamma} \sigma_{\Gamma}(\boldsymbol{w}): \nabla_{\Gamma} \hat{\boldsymbol{w}}
$$

Thus, $\mathrm{D}_{\boldsymbol{u}} \mathcal{F}(\mathbf{0}, \boldsymbol{u})$ is an isomorphism.

Hence, the implicit function theorem applies and then there exists a $C^{1}$ function $\boldsymbol{\theta} \in \mathcal{U} \mapsto$ $\boldsymbol{v}(\boldsymbol{\theta}) \in \mathcal{H}(\Omega \backslash \bar{\omega})$ such that $\mathcal{F}(\mathbf{0}, \boldsymbol{v}(\boldsymbol{\theta}))=\mathbf{0}$ in a neighborhood of $\mathbf{0}$. Using the uniqueness of the solution of such a problem, we obtain that $\boldsymbol{\theta} \in \mathcal{U} \mapsto \boldsymbol{u}^{\theta} \in \mathcal{H}(\Omega \backslash \bar{\omega})$ is $C^{1}$.

Lemma 3.4. There exists $\widetilde{\boldsymbol{u}}_{\theta}$ an extension in $\mathbb{R}^{d}$ of $\boldsymbol{u}_{\theta}$ such that

$$
\boldsymbol{\theta} \in \mathcal{U} \mapsto \widetilde{\boldsymbol{u}}_{\theta} \in \mathbf{L}^{2}\left(\mathbb{R}^{d}\right)
$$

is differentiable at $\mathbf{0}$.

Proof. For $\boldsymbol{\theta} \in \mathcal{U}, \boldsymbol{u}_{\theta}=\mathcal{P}^{*}(\boldsymbol{\theta}) \boldsymbol{u}^{\theta} \in \mathcal{H}\left(\Omega \backslash \overline{\omega_{\theta}}\right) \subset \mathbf{H}^{1}\left(\Omega \backslash \overline{\omega_{\theta}}\right)$. According to the differentiability of $\boldsymbol{\theta} \mapsto \boldsymbol{u}^{\theta}$ (see Lemma 3.3 and Stein's extension theorem (see, e.g., [2, Theorem 5.24]), there exists $\widetilde{\boldsymbol{u}}^{\theta}$, an extension of $\boldsymbol{u}^{\theta}$, such that $\boldsymbol{\theta} \in \mathcal{U} \mapsto \widetilde{\boldsymbol{u}}^{\theta} \in \mathbf{H}^{1}\left(\mathbb{R}^{d}\right)$ is differentiable at $\mathbf{0}$. Moreover,

$$
\boldsymbol{\theta} \in \mathcal{U} \mapsto(\mathbf{I}+\boldsymbol{\theta})^{-1}-\mathbf{I} \in \boldsymbol{C}^{1, \infty}\left(\mathbb{R}^{d}\right) \quad \text { and } \quad \boldsymbol{\theta} \in \mathcal{U} \mapsto\left(\mathrm{I}_{d}+\nabla \boldsymbol{\theta}\right)^{-1} \in \boldsymbol{C}^{1, \infty}\left(\mathbb{R}^{d}\right)
$$

are differentiable at $\mathbf{0}$. Thus,

$$
\mathcal{V}_{1}: \boldsymbol{\theta} \in \mathcal{U} \mapsto\left(\left(\mathrm{I}_{d}+\nabla \boldsymbol{\theta}\right)^{-1} \widetilde{\boldsymbol{u}}^{\theta},(\mathbf{I}+\boldsymbol{\theta})^{-1}-\mathbf{I}\right) \in \mathbf{H}^{1}\left(\mathbb{R}^{d}\right) \times \boldsymbol{C}^{1, \infty}\left(\mathbb{R}^{d}\right)
$$

is differentiable at $\mathbf{0}$. We apply [35, Lemma 5.3.9] to get that

$$
\mathcal{V}_{2}:(\boldsymbol{h}, \boldsymbol{\mu}) \in \mathbf{H}^{1}\left(\mathbb{R}^{d}\right) \times \boldsymbol{C}^{1, \infty}\left(\mathbb{R}^{d}\right) \mapsto \boldsymbol{h} \circ(\mathbf{I}+\boldsymbol{\mu}) \in \mathbf{L}^{2}\left(\mathbb{R}^{d}\right)
$$

is $C^{1}$. By composition, $\boldsymbol{\theta} \in \mathcal{U} \mapsto \widetilde{\boldsymbol{u}}_{\theta}:=\mathcal{V}_{2} \circ \mathcal{V}_{1}(\boldsymbol{\theta}) \in \mathbf{L}^{2}\left(\mathbb{R}^{d}\right)$ is differentiable at $\mathbf{0}$.

Using the Fréchet differentiability given by this lemma, we obtain the Gâteaux differentiability in the direction $\boldsymbol{V} \in \mathcal{U}$ : there exists $\widetilde{\boldsymbol{u}}_{t}$, extension in $\mathbb{R}^{d}$ of $\boldsymbol{u}_{t}$ such that the function $t \in[0, T) \mapsto$ $\widetilde{\boldsymbol{u}}_{t} \in \mathbf{L}^{2}\left(\mathbb{R}^{d}\right)$ is differentiable at 0 by composition. Hence, we prove the following result.

Lemma 3.5. The solution $\boldsymbol{u}$ of Problem (1.4) is differentiable with respect to the domain $\omega \in \mathcal{O}_{\delta}$. We denote by $\boldsymbol{u}^{\prime}$ its derivative at 0 . 


\subsection{Shape derivatives for the GIBC problem (Proof of Theorem 1.3)}

Since we proved in the previous section the differentiability with respect to the domain, we can now compute the shape derivatives of the state. For the reader's convenience, let us recall the definition of the shape derivative in our situation (see [35] for details).

- If the mapping $\boldsymbol{\theta} \in \mathcal{U} \mapsto \boldsymbol{u}_{\theta} \circ(\mathbf{I}+\boldsymbol{\theta}) \in \mathbf{L}^{2}(\Omega \backslash \bar{\omega})$ is Fréchet differentiable at $\mathbf{0}$, we say that $\boldsymbol{\theta} \mapsto \boldsymbol{u}_{\theta}$ possesses a total first variation (or derivative) at $\mathbf{0}$. In such a case, this total first derivative at $\mathbf{0}$ in the direction $\boldsymbol{\theta}$ is denoted by $\dot{\boldsymbol{u}}_{\theta}$.

- If, for every $\mathscr{D} \subset \subset \Omega \backslash \bar{\omega}$, the mapping $\boldsymbol{\theta} \in \mathcal{U} \mapsto \boldsymbol{u}_{\theta \mid \mathscr{D}} \in \mathbf{L}^{2}(\mathscr{D})$ is Fréchet differentiable at $\mathbf{0}$, we say that $\boldsymbol{\theta} \mapsto \boldsymbol{u}_{\theta}$ possesses a local first variation (or derivative) at $\mathbf{0}$. In such a case, this local first derivative at $\mathbf{0}$ in the direction $\boldsymbol{\theta}$ is denoted by $\boldsymbol{u}_{\theta}^{\prime}$, is called shape derivative and is well defined in the whole domain $\Omega \backslash \bar{\omega}$ :

$$
\boldsymbol{u}_{\theta}^{\prime}=\left.\frac{\mathrm{d}}{\mathrm{d} t}\left(\boldsymbol{u}_{t \boldsymbol{\theta} \mid \mathscr{D}}\right)\right|_{t=0} \quad \text { in each } \mathscr{D} \subset \subset \Omega \backslash \bar{\omega} .
$$

In the following, for $\boldsymbol{V} \in \mathcal{U}$, we denote by $\boldsymbol{u}^{\prime}$ the local first variation $\boldsymbol{u}_{V}^{\prime}$ which is referred as the shape derivative of the state. We also use the notation

$$
V_{\mathrm{n}}:=\boldsymbol{V} \cdot \mathbf{n} .
$$

Let $T>0$. For a given perturbation $\boldsymbol{V} \in \mathcal{U}$ and $t \in[0, T)$, we define

$$
\omega_{t}:=(\mathbf{I}+t \boldsymbol{V})(\omega)
$$

with boundary $\Gamma_{t}$ and we consider the solution $\boldsymbol{u}_{t} \in \mathcal{H}\left(\Omega \backslash \overline{\omega_{t}}\right)$ of the following perturbed GIBC problem:

$$
\left\{\begin{aligned}
-\operatorname{div}\left(A_{\text {ext }} e\left(\boldsymbol{u}_{t}\right)\right) & =\boldsymbol{f} & & \text { in } \Omega \backslash \overline{\omega_{t}} \\
\boldsymbol{u}_{t} & =\mathbf{0} & & \text { on } \Gamma_{D} \\
\left(A_{\mathrm{ext}} e\left(\boldsymbol{u}_{t}\right)\right) \mathbf{n} & =\boldsymbol{g} & & \text { on } \Gamma_{N} \\
\left(A_{\mathrm{ext}} e\left(\boldsymbol{u}_{t}\right)\right) \mathbf{n}_{t}-\varepsilon \operatorname{div}_{\Gamma_{t}}\left(\sigma_{\Gamma_{t}}\left(\boldsymbol{u}_{t}\right)\right) & =\mathbf{0} & & \text { on } \Gamma_{t},
\end{aligned}\right.
$$

where $\mathbf{n}_{t}$ represents the exterior unit normal to $\Gamma_{t}$, where

$$
e_{\Gamma_{t}}\left(\boldsymbol{u}_{t}\right):=\frac{1}{2} \Pi_{d, t}\left(\nabla_{\Gamma_{t}} \boldsymbol{u}_{t}+{ }^{t} \nabla_{\Gamma_{t}} \boldsymbol{u}_{t}\right) \Pi_{d, t} \quad \text { and } \quad \Pi_{d, t}:=\mathrm{I}_{d}-\mathbf{n}_{t} \otimes \mathbf{n}_{t}
$$

and where

$$
\sigma_{\Gamma_{t}}\left(\boldsymbol{u}_{t}\right):=\bar{\lambda}_{\mathrm{int}}\left(\operatorname{div}_{\Gamma_{t}} \boldsymbol{u}_{t}\right) \Pi_{d, t}+2 \mu_{\mathrm{int}} e_{\Gamma_{t}}\left(\boldsymbol{u}_{t}\right) .
$$

Proof of Theorem 1.3. For $t \in[0, T)$, we consider Problem (3.5). By differentiating with respect to the shape at $t=0$, we classically obtain (see, e.g., [42, Theorem 3.3] or also [35, Chapter 5])

$$
\left\{\begin{aligned}
-\operatorname{div}\left(A_{\text {ext }} e\left(\boldsymbol{u}^{\prime}\right)\right) & =\mathbf{0} & & \text { in } \Omega \backslash \bar{\omega} \\
\boldsymbol{u}^{\prime} & =\mathbf{0} & & \text { on } \Gamma_{D} \\
\left(A_{\mathrm{ext}} e\left(\boldsymbol{u}^{\prime}\right)\right) \mathbf{n} & =\mathbf{0} & & \text { on } \Gamma_{N} .
\end{aligned}\right.
$$

Let us now compute the shape derivative of the $\operatorname{GIBC}\left(A_{\text {ext }} e\left(\boldsymbol{u}_{t}\right)\right) \mathbf{n}_{t}-\operatorname{div}_{\Gamma_{t}}\left(\sigma_{\Gamma_{t}}\left(\boldsymbol{u}_{t}\right)\right)=\mathbf{0}$ on $\Gamma_{t}$. We define by $\boldsymbol{\tau}_{t}$ the transformation that maps the restriction of $\boldsymbol{u}_{t}$ to $\Gamma_{t}$ onto the restriction of $\boldsymbol{u}_{t} \circ(\mathbf{I}+t \boldsymbol{V})$ to $\Gamma$. Since we know that the shape derivative depends only on the normal component of the variations $\boldsymbol{V}$ (see, e.g., [35, Theorem 5.9.2]), we compute the material derivatives in the direction $\boldsymbol{V}=V_{\mathrm{n}} \mathbf{n}$. We first recall that we have [35, Corrolaire 5.2.5]

Then, we get

$$
\frac{\mathrm{d}}{\mathrm{d} t}\left[\boldsymbol{\tau}_{t} \boldsymbol{u}_{t}\right]_{t=0}=\boldsymbol{u}^{\prime}+V_{\mathrm{n}} \partial_{\mathbf{n}} \boldsymbol{u}
$$

$$
\begin{aligned}
\frac{\mathrm{d}}{\mathrm{d} t}\left[\left(\boldsymbol{\tau}_{t} A_{\mathrm{ext}} e\left(\boldsymbol{u}_{t}\right)\right)\left(\boldsymbol{\tau}_{t} \mathbf{n}_{t}\right)\right]_{t=0} & =\left(A_{\text {ext }} e\left(\boldsymbol{u}^{\prime}\right)\right) \mathbf{n}-\left(A_{\text {ext }} e(\boldsymbol{u})\right) \nabla_{\Gamma} V_{\mathrm{n}}+V_{\mathrm{n}} \partial_{\mathbf{n}}\left(A_{\text {ext }} e(\boldsymbol{u})\right) \mathbf{n} \\
& =\left(A_{\text {ext }} e\left(\boldsymbol{u}^{\prime}\right)\right) \mathbf{n}-\left(A_{\text {ext }} e(\boldsymbol{u})\right) \nabla_{\Gamma} V_{\mathrm{n}}+V_{\mathrm{n}}\left(\operatorname{div}-\operatorname{div}_{\Gamma}\right)\left(A_{\text {ext }} e(\boldsymbol{u})\right) \\
& =\left(A_{\text {ext }} e\left(\boldsymbol{u}^{\prime}\right)\right) \mathbf{n}-\left(A_{\text {ext }} e(\boldsymbol{u})\right) \nabla_{\Gamma} V_{\mathrm{n}}-V_{\mathrm{n}} \boldsymbol{f}-V_{\mathrm{n}} \operatorname{div}{ }_{\Gamma}\left(A_{\text {ext }} e(\boldsymbol{u})\right) \\
& =\left(A_{\text {ext }} e\left(\boldsymbol{u}^{\prime}\right)\right) \mathbf{n}-V_{\mathrm{n}} \boldsymbol{f}-\operatorname{div}_{\Gamma}\left(V_{\mathrm{n}} A_{\text {ext }} e(\boldsymbol{u})\right) .
\end{aligned}
$$


Since $\left(A_{\text {ext }} e(\boldsymbol{u})\right) \mathbf{n}=\operatorname{div}_{\Gamma}\left(\sigma_{\Gamma}(\boldsymbol{u})\right)$, we have

$$
\begin{aligned}
\frac{\mathrm{d}}{\mathrm{d} t}\left[\left(\boldsymbol{\tau}_{t} A_{\text {ext }} e\left(\boldsymbol{u}_{t}\right)\right)\left(\boldsymbol{\tau}_{t} \mathbf{n}_{t}\right)\right]_{t=0}= & \left(A_{\text {ext }} e\left(\boldsymbol{u}^{\prime}\right)\right) \mathbf{n}-V_{\mathrm{n}} \boldsymbol{f}-\operatorname{div}_{\Gamma}\left(V_{\mathrm{n}} \Pi_{d}\left(A_{\text {ext }} e(\boldsymbol{u})\right) \Pi_{d}\right) \\
& -V_{\mathrm{n}} \mathrm{H}\left(A_{\text {ext }} e(\boldsymbol{u})\right) \mathbf{n}-\operatorname{div}_{\Gamma}\left(V_{\mathrm{n}} \Pi_{d}\left(A_{\text {ext }} e(\boldsymbol{u})\right) \mathbf{n} \otimes \mathbf{n}\right) .
\end{aligned}
$$

Moreover, we have (see [22, Theorem 5.4])

$$
\frac{\mathrm{d}}{\mathrm{d} t}\left[\boldsymbol{\tau}_{t} \nabla_{\Gamma_{t}} \boldsymbol{\tau}_{t}^{-1}\right]_{t=0} \boldsymbol{u}=-V_{\mathrm{n}}\left[\mathrm{D}^{2} b \nabla_{\Gamma} \boldsymbol{u}\right]+\mathbf{n} \otimes\left({ }^{t}\left[\nabla_{\Gamma} \boldsymbol{u}\right] \nabla_{\Gamma} V_{\mathrm{n}}\right)
$$

and (see [22, Theorem 5.6])

$$
\begin{aligned}
\frac{\mathrm{d}}{\mathrm{d} t}\left[\boldsymbol{\tau}_{t} \operatorname{div}_{\Gamma_{t}} \boldsymbol{\tau}_{t}^{-1}\right]_{t=0} \boldsymbol{u}= & -V_{\mathrm{n}} \operatorname{Tr}\left[\mathrm{D}^{2} b \nabla_{\Gamma} \boldsymbol{u}\right]+\left[\nabla_{\Gamma} \boldsymbol{u}\right] \mathbf{n} \cdot \nabla_{\Gamma} V_{\mathrm{n}} \\
= & -V_{\mathrm{n}}\left(\operatorname{div}_{\Gamma}\left(\mathrm{D}^{2} b-\mathrm{H} \Pi_{d}\right) \boldsymbol{u}+\operatorname{Hdiv}_{\Gamma} \Pi_{d} \boldsymbol{u}+\operatorname{Tr}\left[\left(\mathrm{D}^{2} b\right)^{2}\right](\boldsymbol{u} \cdot \mathbf{n})\right) \\
& +\nabla_{\Gamma}(\boldsymbol{u} \cdot \mathbf{n}) \cdot \nabla_{\Gamma} V_{\mathrm{n}}-\left[\mathrm{D}^{2} b\right] \boldsymbol{u} \cdot \nabla_{\Gamma} V_{\mathrm{n}} .
\end{aligned}
$$

Then,

$$
\begin{aligned}
\frac{\mathrm{d}}{\mathrm{d} t}\left[\boldsymbol{\tau}_{t} \operatorname{div}_{\Gamma_{t}} \boldsymbol{\tau}_{t}^{-1}\right]_{t=0} \sigma_{\Gamma}(\boldsymbol{u})= & \\
& -V_{\mathrm{n}}\left(\operatorname{div}_{\Gamma}\left(\left(\mathrm{D}^{2} b-\mathrm{H} \Pi_{d}\right) \sigma_{\Gamma}(\boldsymbol{u})\right)+\operatorname{Hdiv}_{\Gamma}\left(\sigma_{\Gamma}(\boldsymbol{u})\right)\right)-{ }^{t}\left(\left[\mathrm{D}^{2} b\right] \sigma_{\Gamma}(\boldsymbol{u})\right) \nabla_{\Gamma} V_{\mathrm{n}} .
\end{aligned}
$$

We have

$$
\begin{aligned}
\sigma_{\Gamma_{t}}(\boldsymbol{u})= & \bar{\lambda}_{\mathrm{int}}\left(\boldsymbol{\tau}_{t} \operatorname{div}_{\Gamma_{t}} \boldsymbol{\tau}_{t}^{-1} \boldsymbol{u}\right)\left(\mathbf{I}-\boldsymbol{\tau}_{t} \mathbf{n}_{t} \otimes \boldsymbol{\tau}_{t} \mathbf{n}_{t}\right) \\
& +2 \mu_{\mathrm{int}}\left(\mathbf{I}-\boldsymbol{\tau}_{t} \mathbf{n}_{t} \otimes \boldsymbol{\tau}_{t} \mathbf{n}_{t}\right)\left(\frac{1}{2}\left(\left[\boldsymbol{\tau}_{t} \nabla_{\Gamma_{t}} \boldsymbol{\tau}_{t}^{-1} \boldsymbol{u}\right]+{ }^{t}\left[\boldsymbol{\tau}_{t} \nabla_{\Gamma_{t}} \boldsymbol{\tau}_{t}^{-1} \boldsymbol{u}\right]\right)\right)\left(\mathbf{I}-\boldsymbol{\tau}_{t} \mathbf{n}_{t} \otimes \boldsymbol{\tau}_{t} \mathbf{n}_{t}\right)
\end{aligned}
$$

and

$$
\frac{\mathrm{d}}{\mathrm{d} t}\left[\left(\mathbf{I}-\boldsymbol{\tau}_{t} \mathbf{n}_{t} \otimes \boldsymbol{\tau}_{t} \mathbf{n}_{t}\right)\right]_{t=0}=\mathbf{n} \otimes \nabla_{\Gamma} V_{\mathrm{n}}+\nabla_{\Gamma} V_{\mathrm{n}} \otimes \mathbf{n} .
$$

Hence we obtain

$$
\begin{aligned}
\frac{\mathrm{d}}{\mathrm{d} t}\left[\sigma_{\Gamma_{t}}\right]_{t=0}(\boldsymbol{u})=-V_{\mathrm{n}}\left(\bar{\lambda}_{\mathrm{int}}\left(\operatorname{Tr}\left[\left[\mathrm{D}^{2} b\right] \nabla_{\Gamma} \boldsymbol{u}\right]\right) \Pi_{d}+2 \mu_{\mathrm{int}} \Pi_{d}\left[\frac{1}{2}\left(\left[\left[\mathrm{D}^{2} b\right] \nabla_{\Gamma} \boldsymbol{u}\right]+{ }^{t}\left[\left[\mathrm{D}^{2} b\right] \nabla_{\Gamma} \boldsymbol{u}\right]\right)\right] \Pi_{d}\right) \\
+\bar{\lambda}_{\mathrm{int}}\left(\left[\nabla_{\Gamma} \boldsymbol{u}\right] \mathbf{n} \cdot \nabla_{\Gamma} V_{\mathrm{n}}\right) \Pi_{d}+\bar{\lambda}_{\mathrm{int}}\left(\operatorname{div}_{\Gamma} \boldsymbol{u}\right)\left(\mathbf{n} \otimes \nabla_{\Gamma} V_{\mathrm{n}}+\nabla_{\Gamma} V_{\mathrm{n}} \otimes \mathbf{n}\right) \\
+2 \mu_{\mathrm{int}}\left[\frac{1}{2}\left(\left[\nabla_{\Gamma} \boldsymbol{u}\right] \mathbf{n} \otimes \nabla_{\Gamma} V_{\mathrm{n}}+\nabla_{\Gamma} V_{\mathrm{n}} \otimes\left[\nabla_{\Gamma} \boldsymbol{u}\right] \mathbf{n}\right)\right] \\
+2 \mu_{\mathrm{int}}\left[\left(\mathbf{n} \otimes\left(e_{\Gamma}(\boldsymbol{u}) \nabla_{\Gamma} V_{\mathrm{n}}\right)+\left(e_{\Gamma}(\boldsymbol{u}) \nabla_{\Gamma} V_{\mathrm{n}}\right) \otimes \mathbf{n}\right)\right]
\end{aligned}
$$

and we deduce

$$
\begin{aligned}
& \operatorname{div}_{\Gamma} \frac{\mathrm{d}}{\mathrm{d} t}\left[\sigma_{\Gamma_{t}}\right]_{t=0}(\boldsymbol{u})= \\
& -\operatorname{div}_{\Gamma}\left(V_{\mathrm{n}}\left(\bar{\lambda}_{\mathrm{int}}\left(\operatorname{Tr}\left[\left[\mathrm{D}^{2} b\right] \nabla_{\Gamma} \boldsymbol{u}\right]\right) \Pi_{d}+2 \mu_{\mathrm{int}} \Pi_{d}\left[\frac{1}{2}\left(\left[\left[\mathrm{D}^{2} b\right] \nabla_{\Gamma} \boldsymbol{u}\right]+{ }^{t}\left[\left[\mathrm{D}^{2} b\right] \nabla_{\Gamma} \boldsymbol{u}\right]\right)\right] \Pi_{d}\right)\right) \\
& +\operatorname{div}_{\Gamma}\left(\bar{\lambda}_{\mathrm{int}}\left(\left[\nabla_{\Gamma} \boldsymbol{u}\right] \mathbf{n} \cdot \nabla_{\Gamma} V_{\mathrm{n}}\right) \Pi_{d}+2 \mu_{\mathrm{int}}\left[\frac{1}{2}\left(\left[\nabla_{\Gamma} \boldsymbol{u}\right] \mathbf{n} \otimes \nabla_{\Gamma} V_{\mathrm{n}}+\nabla_{\Gamma} V_{\mathrm{n}} \otimes\left[\nabla_{\Gamma} \boldsymbol{u}\right] \mathbf{n}\right)\right]\right) \\
& +\left(\mathrm{H}_{d}+\left[\mathrm{D}^{2} b\right]+\operatorname{ndiv}_{\Gamma} \Pi_{d}\right)\left(\sigma_{\Gamma}(\boldsymbol{u}) \nabla_{\Gamma} V_{\mathrm{n}}\right) .
\end{aligned}
$$

We conclude using the chain and product rules that

$$
\begin{aligned}
\frac{\mathrm{d}}{\mathrm{d} t}\left[\left(\boldsymbol{\tau}_{t} A_{\text {ext }} e\left(\boldsymbol{u}_{t}\right)\right)\left(\boldsymbol{\tau}_{t} \mathbf{n}_{t}\right)\right]_{t=0}+\frac{\mathrm{d}}{\mathrm{d} t}\left[\boldsymbol{\tau}_{t} \operatorname{div}_{\Gamma_{t}} \boldsymbol{\tau}_{t}^{-1}\right]_{t=0} \sigma_{\Gamma}(\boldsymbol{u}) \\
+\operatorname{div}_{\Gamma} \frac{\mathrm{d}}{\mathrm{d} t}\left[\sigma_{\Gamma_{t}}\right]_{t=0}(\boldsymbol{u})+\operatorname{div}_{\Gamma} \sigma_{\Gamma}\left(\frac{\mathrm{d}}{\mathrm{d} t}\left[\boldsymbol{\tau}_{t} \boldsymbol{u}_{t}\right]_{t=0}\right)=\mathbf{0} .
\end{aligned}
$$




\subsection{Asymptotic analysis for the shape derivative of the transmission problem}

This section focuses on the asymptotic analysis of the shape derivative of the solution to the thin layer transmission problem 1.2. First we give a characterization of the shape derivative as a solution to a new transmission problem with non-vanishing jumps. The existence of the shape derivative follows from standard procedures (see, e.g., [42, Chapter 3.4]). Hence we claim the following result which characterizes the shape derivative of the state (see, e.g., [42, Theorem 3.3] and [38] for the jump formula).

Theorem 3.6. The thin layer transmission problem characterizing the first shape derivatives in the direction $V$ is

$$
\left\{\begin{aligned}
-\operatorname{div} A_{\mathrm{ext}} e\left(\boldsymbol{u}_{e}^{\prime \varepsilon}\right) & =\mathbf{0} & & \text { in } \Omega \backslash \bar{\omega} \\
-\operatorname{div} A_{\mathrm{int}} e\left(\boldsymbol{u}_{i}^{\prime \varepsilon}\right) & =\mathbf{0} & & \text { in } \omega_{\text {int }}^{\varepsilon} \\
\boldsymbol{u}_{e}^{\prime \varepsilon} & =\mathbf{0} & & \text { on } \Gamma_{D} \\
\left(A_{\mathrm{ext}} e\left(\boldsymbol{u}_{e}^{\prime \varepsilon}\right)\right) \mathbf{n} & =\mathbf{0} & & \text { on } \Gamma_{N} \\
A_{\mathrm{ext}} e\left(\boldsymbol{u}_{e}^{\prime \varepsilon}\right) \mathbf{n}-A_{\mathrm{int}} e\left(\boldsymbol{u}_{i}^{\prime \varepsilon}\right) \mathbf{n} & =V_{\mathrm{n}} \boldsymbol{f}+\operatorname{div}_{\Gamma} V_{\mathrm{n}} \Pi_{d} A_{\mathrm{ext}} e\left(\boldsymbol{u}_{e}^{\varepsilon}\right) \Pi_{d}-\operatorname{div}_{\Gamma} V_{\mathrm{n}} \Pi_{d} A_{\mathrm{int}} e\left(\boldsymbol{u}_{i}^{\varepsilon}\right) \Pi_{d} & & \text { on } \Gamma \\
\boldsymbol{u}_{e}^{\prime \varepsilon}-\boldsymbol{u}_{i}^{\prime \varepsilon} & =-V_{\mathrm{n}}\left(\partial_{\mathrm{n}} \boldsymbol{u}_{e}^{\varepsilon}-\partial_{\mathrm{n}} \boldsymbol{u}_{i}^{\varepsilon}\right) & & \text { on } \Gamma \\
A_{\mathrm{int}} e\left(\boldsymbol{u}_{i}^{\prime \varepsilon}\right) \mathbf{n}^{\varepsilon} & =\operatorname{div}_{\Gamma}^{\varepsilon} \boldsymbol{\tau}_{\varepsilon}^{-1}\left(V_{\mathrm{n}}\right)\left(\bar{A}_{\mathrm{int}} e_{\Gamma}^{\varepsilon}\left(\boldsymbol{u}_{i}^{\varepsilon}\right)\right) & & \text { on } \Gamma^{\varepsilon} .
\end{aligned}\right.
$$

Then, for $N=0,1$, we want to approximate the shape derivative $\boldsymbol{u}^{\prime}$, solution of Problem (3.6), by the solution $\boldsymbol{w}_{[N]}^{\varepsilon}$ of some boundary value problems of the form

$$
\left\{\begin{array}{rlll}
-\operatorname{div} A_{\mathrm{ext}} e\left(\boldsymbol{w}_{[N]}^{\varepsilon}\right) & =\mathbf{0} & & \text { in } \Omega \backslash \bar{\omega} \\
\boldsymbol{w}_{[N]}^{\varepsilon} & =\mathbf{0} & \text { on } \Gamma_{D} \\
\left(A_{\mathrm{ext}} e\left(\boldsymbol{w}_{[N]}^{\varepsilon}\right)\right) \mathbf{n} & =\mathbf{0} & & \text { on } \Gamma_{N} \\
C\left(\varepsilon, A_{\mathrm{ext}} e\left(\boldsymbol{w}_{[N]}^{\varepsilon}\right) \mathbf{n}, \boldsymbol{w}_{[N]}^{\varepsilon}\right) & =\boldsymbol{h} & & \text { on } \Gamma
\end{array}\right.
$$

where $C\left(\varepsilon, A_{\text {ext }} e\left(\boldsymbol{w}_{[N]}^{\varepsilon}\right) \mathbf{n}, \boldsymbol{w}_{[N]}^{\varepsilon}\right)$ is a so-called Generalized Impedance Boundary Condition (GIBC). The following result gives the GIBC modeling an approximation up to $O\left(\varepsilon^{N+1}\right)$ of $\boldsymbol{u}_{e}^{\prime \varepsilon}$ for $N=0,1$. We recall that $\boldsymbol{u}_{[0]}^{\varepsilon}:=\boldsymbol{u}_{e}^{0}$ and $\boldsymbol{u}_{[1]}^{\varepsilon}:=\boldsymbol{u}_{e}^{0}+\varepsilon \boldsymbol{u}_{e}^{1}$ (see Section 2).

Proposition 3.7. The GIBC, defined on $\Gamma$, modeling the interior thin layer effects of the inclusion $\omega$ on the first shape derivative for $N=0,1$, are given by $C\left(\varepsilon, A_{\text {ext }} e\left(\boldsymbol{w}_{[N]}^{\varepsilon}\right) \mathbf{n}, \boldsymbol{w}_{[N]}^{\varepsilon}\right)=\boldsymbol{h}$ with

$$
C\left(\varepsilon, A_{\mathrm{ext}} e\left(\boldsymbol{w}_{[N]}^{\varepsilon}\right) \mathbf{n}, \boldsymbol{w}_{[N]}^{\varepsilon}\right):=\left(A_{\mathrm{ext}} e\left(\boldsymbol{w}_{[N]}^{\varepsilon}\right) \mathbf{n}\right)+\mathbf{C}^{\varepsilon, N} \boldsymbol{w}_{[N]}^{\varepsilon},
$$

where $\mathbf{C}^{\varepsilon, 0}:=\mathbf{0}$ and $\mathbf{C}^{\varepsilon, 1} \boldsymbol{w}:=-\varepsilon \operatorname{div}_{\Gamma}\left(\bar{A}_{\text {int }} e_{\Gamma}(\boldsymbol{w})\right)$, and with

$$
\boldsymbol{h}:=V_{\mathrm{n}} \boldsymbol{f}+\mathbf{F}_{1}^{\varepsilon, N}
$$

$$
\begin{aligned}
& \text { where } \mathbf{F}_{1}^{\varepsilon, 0}:=\operatorname{div}_{\Gamma}\left(V_{\mathrm{n}} \Pi_{d}\left(A_{\mathrm{ext}} e\left(\boldsymbol{u}_{[0]}^{\varepsilon}\right)\right) \Pi_{d}\right)=\operatorname{div}_{\Gamma} V_{\mathrm{n}}\left(\bar{A}_{\mathrm{ext}} e_{\Gamma}\left(\boldsymbol{u}_{[0]}^{\varepsilon}\right)\right) \text { and } \\
& \begin{array}{r}
\mathbf{F}_{1}^{\varepsilon, 1}:=\operatorname{div}_{\Gamma}\left(V_{\mathrm{n}} \Pi_{d}\left(A_{\mathrm{ext}} e\left(\boldsymbol{u}_{[1]}^{\varepsilon}\right)\right) \Pi_{d}\right)-\varepsilon \operatorname{div}_{\Gamma}\left(V_{\mathrm{n}}\left(\left[\mathrm{D}^{2} b\right]-\operatorname{H} \Pi_{d}\right)\left(\bar{A}_{\mathrm{int}} e_{\Gamma}\left(\boldsymbol{u}_{[1]}^{\varepsilon}\right)\right)\right) \\
-\varepsilon \operatorname{div}_{\Gamma}\left(V_{\mathrm{n}}\left(\bar{A}_{\mathrm{int}}\left[\frac{1}{2} \Pi_{d}\left(\left[\left[\mathrm{D}^{2} b\right] \nabla_{\Gamma} \boldsymbol{u}_{[1]}^{\varepsilon}\right]+{ }^{t}\left[\left[\mathrm{D}^{2} b\right] \nabla_{\Gamma} \boldsymbol{u}_{[1]}^{\varepsilon}\right]\right) \Pi_{d}\right]\right)\right) \\
+\varepsilon \operatorname{div}_{\Gamma}\left(\bar{A}_{\mathrm{int}} e_{\Gamma}\left(V_{\mathrm{n}}\left(\partial_{\mathbf{n}} \boldsymbol{u}_{[1]}^{\varepsilon}-\Lambda_{0}^{-1} A_{\mathrm{ext}} e\left(\boldsymbol{u}_{[1]}^{\varepsilon}\right) \mathbf{n}\right)\right)\right) \\
+\varepsilon\left(\left[\mathrm{D}^{2} b\right]+\mathbf{n} \operatorname{div}_{\Gamma} \Pi_{d}\right) \operatorname{div}_{\Gamma}\left(V_{\mathrm{n}}\left(\bar{A}_{\mathrm{int}} e_{\Gamma}\left(\boldsymbol{u}_{[1]}^{\varepsilon}\right)\right)\right) \\
\quad+\varepsilon \operatorname{div}_{\Gamma}\left(\bar{A}_{\mathrm{int}}\left[\frac{1}{2}\left(\left[\nabla_{\Gamma} \boldsymbol{u}_{[1]}^{\varepsilon}\right] \mathbf{n} \otimes \nabla_{\Gamma} V_{\mathrm{n}}+\nabla_{\Gamma} V_{\mathrm{n}} \otimes\left[\nabla_{\Gamma} \boldsymbol{u}_{[1]}^{\varepsilon}\right] \mathbf{n}\right)\right]\right) .
\end{array}
\end{aligned}
$$


Proof. We set $\boldsymbol{u}_{e}^{\prime \varepsilon}(x)=\sum_{n \geq 0} \varepsilon^{n} \boldsymbol{u}_{e}^{n}(x)$ in $\Omega \backslash \bar{\omega}$ and $\boldsymbol{u}_{i}^{\prime \varepsilon}(x, s)=\sum_{n \geq 0} \varepsilon^{n} \mathbf{U}_{i}^{n}(x, S)$ in $\Gamma \times[0,1]$. Following the computations given in Section 2 (see also [37]), we obtain

$$
\begin{aligned}
\mathbf{U}_{i}^{0}(\cdot, S)= & \boldsymbol{u}_{e}^{0}+V_{\mathrm{n}}\left(\partial_{\mathrm{n}} \boldsymbol{u}_{e}^{0}+\Lambda_{0}^{-1}\left(\mathbf{B}_{t}^{0} \boldsymbol{u}_{e}^{0}-A_{\mathrm{ext}} e\left(\boldsymbol{u}_{e}^{0}\right) \mathbf{n}\right)\right), \\
\mathbf{U}_{i}^{1}(\cdot, S)=\quad-S \Lambda_{0}^{-1} \mathbf{B}_{t}^{0} \mathbf{U}_{i}^{0}(\cdot, 0)+S \Lambda_{0}^{-1} \operatorname{div}_{\Gamma}\left(V_{\mathrm{n}}\left(\bar{A}_{\mathrm{int}} e_{\Gamma}\left(\boldsymbol{u}_{e}^{0}\right)\right)\right) & +\boldsymbol{u}_{e}^{1}+V_{\mathrm{n}}\left(\partial_{\mathrm{n}} \boldsymbol{u}_{e}^{1}+\Lambda_{0}^{-1}\left(\mathbf{B}_{t}^{0} \boldsymbol{u}_{e}^{1}-A_{\mathrm{ext}}\left(\boldsymbol{u}_{e}^{1}\right) \mathbf{n}\right)\right),
\end{aligned}
$$

and

$$
\begin{gathered}
\Lambda_{0} \mathbf{U}_{i}^{2}(\cdot, S)=\left(\frac{S^{2}}{2}-S\right)\left[\left(\Lambda_{1} \Lambda_{0}^{-1} \mathbf{B}_{t}^{0}-\Lambda_{2,2}\right) \mathbf{U}_{i}^{0}(\cdot, 0)-\Lambda_{1} \Lambda_{0}^{-1} \operatorname{div}_{\Gamma}\left(V_{\mathrm{n}}\left(\bar{A}_{\mathrm{int}} e_{\Gamma}\left(\boldsymbol{u}_{e}^{1}\right)\right)\right)\right] \\
+S\left[\left(\mathbf{B}_{t}^{0} \Lambda_{0}^{-1} \mathbf{B}_{t}^{0}-\mathbf{B}_{t}^{1}\right) \mathbf{U}_{i}^{0}(\cdot, 0)-\mathbf{B}_{t}^{0} \Lambda_{0}^{-1} \operatorname{div}_{\Gamma}\left(V_{\mathrm{n}}\left(\bar{A}_{\mathrm{int}} e_{\Gamma}\left(\boldsymbol{u}_{e}^{1}\right)\right)\right)\right. \\
-\operatorname{div}_{\Gamma}\left(V_{\mathrm{n}}\left(\bar{A}_{\mathrm{int}} e_{\Gamma}\left(\Lambda_{0}^{-1} \mathbf{B}_{t}^{0} \boldsymbol{u}_{e}^{0}-\boldsymbol{u}_{e}^{1}\right)\right)\right)-\operatorname{div}_{\Gamma}\left(V_{\mathrm{n}}\left(\left[\mathrm{D}^{2} b\right]-\mathrm{H} \Pi_{d}\right)\left(\bar{A}_{\mathrm{int}} e_{\Gamma}\left(\boldsymbol{u}_{e}^{0}\right)\right)\right) \\
\left.-\operatorname{Hdiv}_{\Gamma}\left(V_{\mathrm{n}}\left(\bar{A}_{\mathrm{int}} e_{\Gamma}\left(\boldsymbol{u}_{e}^{0}\right)\right)\right)-\operatorname{div}_{\Gamma}\left(V_{\mathrm{n}}\left(\bar{A}_{\mathrm{int}}\left[\frac{1}{2} \Pi_{d}\left(\left[\left[\mathrm{D}^{2} b\right] \nabla_{\Gamma} \boldsymbol{u}_{e}^{0}\right]+{ }^{t}\left[\left[\mathrm{D}^{2} b\right] \nabla_{\Gamma} \boldsymbol{u}_{e}^{0}\right]\right) \Pi_{d}\right]\right)\right)-\mathbf{B}_{t}^{0} \mathbf{U}_{i}^{1}(\cdot, 0)\right] .
\end{gathered}
$$

For $N=0$, we have

$$
\begin{aligned}
A_{\mathrm{ext}} e\left(\boldsymbol{u}_{e}^{0}\right) \mathbf{n}= & \partial_{S} \Lambda_{0} \mathbf{U}_{i}^{1}(x, 0)+\mathbf{B}_{t}^{0} \mathbf{U}_{i}^{0}(x, 0)+V_{\mathrm{n}} \boldsymbol{f}+\operatorname{div}_{\Gamma} V_{\mathrm{n}} \Pi_{d} A_{\mathrm{ext}} e\left(\boldsymbol{u}_{e}^{0}\right) \Pi_{d} \\
\quad & \quad-\operatorname{div}_{\Gamma}\left(V_{\mathrm{n}}\left(\bar{A}_{\mathrm{int}} e_{\Gamma}\left(\boldsymbol{u}_{e}^{0}\right)\right)-\frac{\lambda_{\mathrm{int}}}{\lambda_{\mathrm{int}}+2 \mu_{\mathrm{int}}} \operatorname{div}_{\Gamma}\left(V_{\mathrm{n}}\left(\mathbf{n} \cdot A_{\mathrm{ext}} e\left(\boldsymbol{u}_{e}^{0}\right) \mathbf{n}\right) \Pi_{d}\right)\right. \\
= & V_{\mathrm{n}} \boldsymbol{f}+\operatorname{div}_{\Gamma} V_{\mathrm{n}} \Pi_{d} A_{\mathrm{ext}} e\left(\boldsymbol{u}_{e}^{0}\right) \Pi_{d} .
\end{aligned}
$$

In this case we approximate $\boldsymbol{u}_{e}^{\prime \varepsilon}$ by $\boldsymbol{u}_{e}^{0}$ and we obtain the GIBC satisfied by $\boldsymbol{w}_{[0]}^{\varepsilon}$.

For $N=1$, we have

$$
\begin{aligned}
A_{\mathrm{ext}} e\left(\boldsymbol{u}_{e}^{1}\right) \mathbf{n}=\partial_{S} \Lambda_{0} \mathbf{U}_{i}^{2}(x, 0)+\mathbf{B}_{t}^{0} \mathbf{U}_{i}^{1}(x, 0)+\operatorname{div}_{\Gamma} V_{\mathrm{n}} \Pi_{d} A_{\mathrm{ext}} e\left(\boldsymbol{u}_{e}^{1}\right) \Pi_{d} \\
\quad-\operatorname{div}_{\Gamma}\left(V_{\mathrm{n}}\left(\Pi_{d} \overline{A_{\mathrm{int}}} e_{\Gamma}\left(\boldsymbol{u}_{e}^{1}\right) \Pi_{d}\right)\right)-\frac{\lambda_{\mathrm{int}}}{\lambda_{\mathrm{int}}+2 \mu_{\mathrm{int}}} \operatorname{div}_{\Gamma}\left(V_{\mathrm{n}}\left(\mathbf{n} \cdot A_{\mathrm{ext}} e\left(\boldsymbol{u}_{e}^{1}\right) \mathbf{n}\right) \Pi_{d}\right) \\
=\operatorname{div}_{\Gamma} V_{\mathrm{n}} \Pi_{d} A_{\mathrm{ext}} e\left(\boldsymbol{u}_{e}^{1}\right) \Pi_{d}-\frac{\lambda_{\mathrm{int}}}{\lambda_{\mathrm{int}}+2 \mu_{\mathrm{int}}} \operatorname{div}_{\Gamma}\left(V_{\mathrm{n}}\left(\mathbf{n} \cdot A_{\mathrm{ext}} e\left(\boldsymbol{u}_{e}^{1}\right) \mathbf{n}\right) \Pi_{d}\right) \\
\quad+\operatorname{div}_{\Gamma}\left(\bar{A}_{\mathrm{int}} e_{\Gamma}\left(\boldsymbol{u}_{e}^{0}+V_{\mathrm{n}}\left(\frac{\partial \boldsymbol{u}_{e}^{0}}{\partial \mathbf{n}}-\Lambda_{0}^{-1} A_{\mathrm{ext}} e\left(\boldsymbol{u}_{e}^{0}\right) \mathbf{n}\right)\right)\right) \\
\left.\left.\quad+\operatorname{div}_{\Gamma}\left(\bar{A}_{\mathrm{int}} e_{\Gamma}\left(V_{\mathrm{n}} \Lambda_{0}^{-1} \mathbf{B}_{t}^{0} \boldsymbol{u}_{e}^{0}\right)\right)\right)-\operatorname{div}_{\Gamma}\left(V_{\mathrm{n}} \bar{A}_{\mathrm{int}} e_{\Gamma}\left(\Lambda_{0}^{-1} \mathbf{B}_{t}^{0} \boldsymbol{u}_{e}^{0}\right)\right)\right) \\
\quad-\operatorname{div}_{\Gamma}\left(V_{\mathrm{n}}\left(\left[\mathrm{D}^{2} b\right]-\mathrm{H} \Pi_{d}\right)\left(\bar{A}_{\mathrm{int}} e_{\Gamma}\left(\boldsymbol{u}_{e}^{0}\right)\right)\right) \\
+\left(\Lambda_{1} \Lambda_{0}^{-1}-\mathbf{B}_{t}^{0} \Lambda_{0}^{-1}-\mathrm{HI}_{d}\right) \operatorname{div}_{\Gamma}\left(V_{\mathrm{n}}\left(\overline{A_{\mathrm{int}}} e_{\Gamma}\left(\boldsymbol{u}_{e}^{0}\right)\right)\right) \\
-\operatorname{div}_{\Gamma}\left(V_{\mathrm{n}}\left(\bar{A}_{\mathrm{int}}\left[\frac{1}{2} \Pi_{d}\left(\left[\left[\mathrm{D}^{2} b\right] \nabla_{\Gamma} \boldsymbol{u}_{e}^{0}\right]+{ }^{t}\left[\left[\mathrm{D}^{2} b\right] \nabla_{\Gamma} \boldsymbol{u}_{e}^{0}\right]\right) \Pi_{d}\right]\right)\right) .
\end{aligned}
$$

Moreover,

$$
\begin{aligned}
\left(\Lambda_{1} \Lambda_{0}^{-1}-\mathbf{B}_{t}^{0} \Lambda_{0}^{-1}-\mathrm{HI}_{d}\right) & =\left(\left[\mathrm{D}^{2} b\right]+\mathbf{n d i v}_{\Gamma} \Pi_{d}\right)-\frac{\lambda_{\mathrm{int}}}{\lambda_{\mathrm{int}}+2 \mu_{\mathrm{int}}}\left(\mathrm{H} \mathbf{n} \otimes \mathbf{n}-\nabla_{\Gamma}(\mathbf{n} \cdot)\right), \\
\mathbf{n} \cdot \operatorname{div}_{\Gamma}\left(V_{\mathrm{n}}\left(\bar{A}_{\mathrm{int}} e_{\Gamma}\left(\boldsymbol{u}_{e}^{0}\right)\right)\right) & =V_{\mathrm{n}} \mathbf{n} \cdot \operatorname{div}_{\Gamma}\left(\left(\bar{A}_{\mathrm{int}} e_{\Gamma}\left(\boldsymbol{u}_{e}^{0}\right)\right)\right)=V_{\mathrm{n}} \mathbf{n} \cdot A_{\mathrm{ext}} e\left(\boldsymbol{u}_{e}^{1}\right) \mathbf{n} .
\end{aligned}
$$

Then

$$
\begin{aligned}
\frac{\lambda_{\text {int }}}{\lambda_{\text {int }}+2 \mu_{\text {int }}} \operatorname{div}_{\Gamma}\left(V_{\mathrm{n}}\left(\mathbf{n} \cdot A_{\text {ext }} e\left(\boldsymbol{u}_{e}^{1}\right) \mathbf{n}\right) \Pi_{d}\right) \\
\quad+\frac{\lambda_{\text {int }}}{\lambda_{\text {int }}+2 \mu_{\text {int }}}\left(\mathrm{H} \mathbf{n} \otimes \mathbf{n}-\nabla_{\Gamma}(\mathbf{n} \cdot)\right) \operatorname{div}_{\Gamma}\left(V_{\mathrm{n}}\left(\bar{A}_{\text {int }} e_{\Gamma}\left(\boldsymbol{u}_{e}^{0}\right)\right)\right)=\mathbf{0},
\end{aligned}
$$

and

$$
\begin{aligned}
\operatorname{div}_{\Gamma}\left(\bar{A}_{\mathrm{int}} e_{\Gamma}\left(V_{\mathrm{n}} \Lambda_{0}^{-1} \mathbf{B}_{t}^{0} \boldsymbol{u}_{e}^{0}\right)\right)-\operatorname{div}_{\Gamma}\left(V_{\mathrm{n}} \bar{A}_{\mathrm{int}} e_{\Gamma}\left(\Lambda_{0}^{-1} \mathbf{B}_{t}^{0} \boldsymbol{u}_{e}^{0}\right)\right) \\
\quad=\operatorname{div}_{\Gamma}\left(\bar{A}_{\mathrm{int}}\left[\frac{1}{2}\left(\left[\nabla_{\Gamma} \boldsymbol{u}_{e}^{0}\right] \mathbf{n} \otimes \nabla_{\Gamma} V_{\mathrm{n}}+\nabla_{\Gamma} V_{\mathrm{n}} \otimes\left[\nabla_{\Gamma} \boldsymbol{u}_{e}^{0}\right] \mathbf{n}\right)\right]\right) .
\end{aligned}
$$

In this case we approximate $\boldsymbol{u}_{e}^{\prime \varepsilon}$ by $\boldsymbol{u}_{e}^{0}+\varepsilon \boldsymbol{u}_{e}^{1}$ and we obtain the GIBC satisfied by $\boldsymbol{w}_{[1]}^{\varepsilon}$. 
We focus now on the remainder $\boldsymbol{r}_{[N]}^{\varepsilon}:=\boldsymbol{v}_{[N]}^{\varepsilon}-\boldsymbol{w}_{[N]}^{\varepsilon}$ for $N=0,1$, where $\boldsymbol{v}_{[N]}^{\prime \varepsilon}$ is the shape derivative of the solution $\boldsymbol{v}_{[N]}^{\varepsilon}$ of Problem 2.1]. We obtain the following convergence result which claims (as underlined in [37]) that one can interchange the asymptotic analysis and the shape derivative calculus.

Theorem 3.8. Let $N=0,1$. There exists a constant $C$ independent on $\varepsilon$ such that

$$
\left\|\boldsymbol{r}_{[N]}^{\varepsilon}\right\|_{\mathbf{L}^{2}(\Omega \backslash \bar{\omega})} \leq C \varepsilon^{N+1} .
$$

Proof. The result is obvious for $N=0$. For $N=1$, we recall that the shape derivative $\boldsymbol{v}_{[N]}^{\prime \varepsilon}$ satisfies the boundary condition:

$$
\left(A_{\mathrm{ext}} e\left(\boldsymbol{v}_{[1]}^{\varepsilon}\right) \mathbf{n}\right)+\mathbf{C}^{\varepsilon, N} \boldsymbol{v}_{[1]}^{\ell}=V_{\mathrm{n}} \boldsymbol{f}+\mathbf{F}_{2}^{\varepsilon, 1}
$$

where

$$
\begin{array}{r}
\mathbf{F}_{2}^{\varepsilon, 1}:=\operatorname{div}_{\Gamma}\left(V_{\mathrm{n}} \Pi_{d}\left(A_{\mathrm{ext}} e\left(\boldsymbol{v}_{[1]}^{\varepsilon}\right)\right) \Pi_{d}\right)+\varepsilon \operatorname{div}_{\Gamma}\left(\bar{A}_{\mathrm{int}} e_{\Gamma}\left(V_{\mathrm{n}}\left(\partial_{\mathbf{n}} \boldsymbol{v}_{[1]}^{\varepsilon}\right)\right)\right) \\
-\varepsilon \operatorname{div}_{\Gamma}\left(V_{\mathrm{n}}\left(\bar{A}_{\mathrm{int}}\left[\frac{1}{2} \Pi_{d}\left(\left[\left[\mathrm{D}^{2} b\right] \nabla_{\Gamma} \boldsymbol{v}_{[1]}^{\varepsilon}\right]+{ }^{t}\left[\left[\mathrm{D}^{2} b\right] \nabla_{\Gamma} \boldsymbol{v}_{[1]}^{\varepsilon}\right]\right) \Pi_{d}\right]\right)\right) \\
-\varepsilon \operatorname{div}_{\Gamma}\left(V_{\mathrm{n}}\left(\left[\mathrm{D}^{2} b\right]-\mathrm{H} \Pi_{d}\right)\left(\bar{A}_{\mathrm{int}} e_{\Gamma}\left(\boldsymbol{v}_{[1]}^{\varepsilon}\right)\right)\right)+\varepsilon\left(\left[\mathrm{D}^{2} b\right]+\mathbf{n} \operatorname{div}_{\Gamma} \Pi_{d}\right) \operatorname{div}_{\Gamma}\left(V_{\mathrm{n}}\left(\bar{A}_{\mathrm{int}} e_{\Gamma}\left(\boldsymbol{v}_{[1]}^{\varepsilon}\right)\right)\right) \\
+\varepsilon \operatorname{div} \Gamma\left(\bar{A}_{\mathrm{int}}\left[\frac{1}{2}\left(\left[\nabla_{\Gamma} \boldsymbol{v}_{[1]}^{\varepsilon}\right] \mathbf{n} \otimes \nabla_{\Gamma} V_{\mathrm{n}}+\nabla_{\Gamma} V_{\mathrm{n}} \otimes\left[\nabla_{\Gamma} \boldsymbol{v}_{[1]}^{\varepsilon}\right] \mathbf{n}\right)\right]\right) .
\end{array}
$$

We easily obtain $\mathbf{F}_{2}^{\varepsilon, 1}-\mathbf{F}_{1}^{\varepsilon, 1}=\varepsilon \operatorname{div}_{\Gamma}\left(\bar{A}_{\text {int }} e_{\Gamma}\left(V_{\mathrm{n}} \Lambda_{0}^{-1} A_{\text {ext }} e\left(\boldsymbol{v}_{[1]}^{\varepsilon}\right) \mathbf{n}\right)\right)$ and using the GIBC satisfied by $\boldsymbol{v}_{[1]}^{\varepsilon}$, we conclude $\mathbf{F}_{1}^{\varepsilon, 1}-\mathbf{F}_{2}^{\varepsilon, 1}=O\left(\varepsilon^{2}\right)$. The estimate results from Lax-Milgram theorem and the Korn's inequality.

Remark 3.9. For $N=1$, we have $\boldsymbol{v}_{[1]}^{\varepsilon}=\boldsymbol{u}$ and $\boldsymbol{v}_{[1]}^{\prime \varepsilon}=\boldsymbol{u}^{\prime}$. Thus the above theorem claims that

$$
\left\|\boldsymbol{u}^{\prime}-\boldsymbol{w}_{[1]}^{\varepsilon}\right\|_{\mathbf{L}^{2}(\Omega \backslash \bar{\omega})} \leq C \varepsilon^{2} .
$$

One can also notice that, in the previous proof, $\mathbf{F}_{2}^{\varepsilon, 1}=\xi\left(\boldsymbol{u}, V_{\mathrm{n}}\right)$ where $\xi\left(\boldsymbol{u}, V_{\mathrm{n}}\right)$ is given in Theorem 1.3 .

\section{Minimization of the compliance}

This part aims to show an application of the previous analysis on the classical problem of the minimization of the compliance of an elastic structure. We recall that the compliance of a structure is the work of the exterior forces which is here given by

$$
\mathcal{J}(\Omega \backslash \bar{\omega}):=\int_{\Omega \backslash \bar{\omega}} A_{\text {ext }} e(\boldsymbol{u}): e(\boldsymbol{u}),
$$

where $\boldsymbol{u} \in \mathcal{H}(\Omega \backslash \bar{\omega})$ solves Problem (1.4). We want to minimize the compliance (that is to say maximise the rigidity of the structure) adding a penalization on the total mass of the structure. Hence we want to minimize the following functional

$$
J(\Omega \backslash \bar{\omega}):=\mathcal{J}(\Omega \backslash \bar{\omega})+\ell|\Omega \backslash \bar{\omega}|
$$

on the admissible sets $\mathcal{O}_{\delta}$, where $\ell>0$ is a given constant of penalization. In other words, $\Omega$ is fixed and known and we want to find the best hole $\omega$ :

$$
\omega^{*} \in \underset{\omega \in \mathcal{O}_{\delta}}{\operatorname{argmin}} J(\Omega \backslash \bar{\omega}) .
$$

To do this, we will use the geometric shape optimization method and make some numerical reconstruction using a classical shape gradient algorithm. Hence we first prove the characterization of the shape gradient of $\mathcal{J}$ given in Theorem 1.4 and then perform some numerical simulations using Freefem ++ (see [34]). 


\subsection{Shape derivative of the compliance (Proof of Theorem 1.4)}

Using the variational formulation of Problem 1.4 with $\boldsymbol{u}$ as a test function, we obtain the following more convenient expression of the objective function:

$$
\mathcal{J}(\Omega \backslash \bar{\omega}):=\int_{\Omega \backslash \bar{\omega}} \boldsymbol{f} \cdot \boldsymbol{u}+\int_{\Gamma_{N}} \boldsymbol{g} \cdot \boldsymbol{u}+\int_{\Gamma}\left(A_{\mathrm{ext}} e(\boldsymbol{u}) \mathbf{n}\right) \cdot \boldsymbol{u} .
$$

Then we can prove Theorem 1.4

Proof of Theorem 1.4. In a first step, we compute the shape derivative of the cost functional. We have (see [35, Eq. (5.4)] and [35, Eq. (5.44)])

$$
\begin{aligned}
\nabla \mathcal{J}(\Omega \backslash \bar{\omega}) \cdot \boldsymbol{V}= & \int_{\Omega \backslash \bar{\omega}} \boldsymbol{f} \cdot \boldsymbol{u}^{\prime}+\int_{\Gamma} V_{\mathrm{n}} \boldsymbol{f} \cdot \boldsymbol{u}+\int_{\Gamma_{N}} \boldsymbol{g} \cdot \boldsymbol{u}^{\prime}+\int_{\Gamma}\left(\left(A_{\mathrm{ext}} e(\boldsymbol{u}) \mathbf{n}\right) \cdot \boldsymbol{u}^{\prime}+\boldsymbol{u} \cdot\left(A_{\mathrm{ext}} e\left(\boldsymbol{u}^{\prime}\right) \mathbf{n}\right)\right) \\
& +\int_{\Gamma}\left(-\boldsymbol{u} \cdot\left(A_{\mathrm{ext}} e(\boldsymbol{u}) \nabla_{\Gamma} V_{\mathrm{n}}\right)+V_{\mathrm{n}} \partial_{\mathbf{n}}\left(\boldsymbol{u} \cdot\left(A_{\mathrm{ext}} e(\boldsymbol{u}) \mathbf{n}\right)\right)+\mathrm{H} V_{\mathrm{n}} \boldsymbol{u} \cdot\left(A_{\mathrm{ext}} e(\boldsymbol{u}) \mathbf{n}\right)\right) \\
= & \int_{\Omega \backslash \bar{\omega}} \boldsymbol{f} \cdot \boldsymbol{u}^{\prime}+\int_{\Gamma} V_{\mathrm{n}} \boldsymbol{f} \cdot \boldsymbol{u}+\int_{\Gamma_{N}} \boldsymbol{g} \cdot \boldsymbol{u}^{\prime}+\int_{\Gamma}\left(\left(A_{\mathrm{ext}} e(\boldsymbol{u}) \mathbf{n}\right) \cdot \boldsymbol{u}^{\prime}+\boldsymbol{u} \cdot\left(A_{\mathrm{ext}} e\left(\boldsymbol{u}^{\prime}\right) \mathbf{n}\right)\right) \\
& -\int_{\Gamma} \boldsymbol{u} \cdot\left(A_{\mathrm{ext}} e(\boldsymbol{u}) \nabla_{\Gamma} V_{\mathrm{n}}\right)+\int_{\Gamma} V_{\mathrm{n}}\left(\left(\partial_{\mathbf{n}} \boldsymbol{u}\right) \cdot\left(A_{\mathrm{ext}} e(\boldsymbol{u}) \mathbf{n}\right)+\boldsymbol{u} \cdot \partial_{\mathrm{n}}\left(A_{\mathrm{ext}} e(\boldsymbol{u})\right) \mathbf{n}\right) \\
& +\int_{\Gamma} \mathrm{H} V_{\mathrm{n}}\left(A_{\mathrm{ext}} e(\boldsymbol{u}) \mathbf{n}\right) \cdot \boldsymbol{u}
\end{aligned}
$$

noticing that $\partial_{\mathbf{n}} \mathbf{n}=0$ (see, e.g., [35, Eq. (5.66)]). We use the following equality (see, e.g., 35, Proposition 5.4.9] $) \partial_{\mathbf{n}}\left(A_{\mathrm{ext}} e(\boldsymbol{u})\right) \mathbf{n}=\operatorname{div}\left(A_{\mathrm{ext}} e(\boldsymbol{u})\right)-\operatorname{div}_{\Gamma}\left(\Pi_{d}\left(A_{\mathrm{ext}} e(\boldsymbol{u})\right)\right)-\mathrm{H}\left(A_{\mathrm{ext}} e(\boldsymbol{u}) \mathbf{n}\right)$ and obtain

$$
\begin{aligned}
\nabla \mathcal{J}(\Omega \backslash \bar{\omega}) \cdot \boldsymbol{V}= & \int_{\Omega \backslash \bar{\omega}} \boldsymbol{f} \cdot \boldsymbol{u}^{\prime}+\int_{\Gamma} V_{\mathrm{n}} \boldsymbol{f} \cdot \boldsymbol{u}+\int_{\Gamma_{N}} \boldsymbol{g} \cdot \boldsymbol{u}^{\prime}+\int_{\Gamma}\left(\left(A_{\mathrm{ext}} e(\boldsymbol{u}) \mathbf{n}\right) \cdot \boldsymbol{u}^{\prime}+\boldsymbol{u} \cdot\left(A_{\mathrm{ext}} e\left(\boldsymbol{u}^{\prime}\right) \mathbf{n}\right)\right) \\
& \quad-\int_{\Gamma} \boldsymbol{u} \cdot\left(A_{\mathrm{ext}} e(\boldsymbol{u}) \nabla_{\Gamma} V_{\mathrm{n}}\right)+\int_{\Gamma} V_{\mathrm{n}}\left(\partial_{\mathbf{n}} \boldsymbol{u}\right) \cdot\left(A_{\mathrm{ext}} e(\boldsymbol{u}) \mathbf{n}\right) \\
& \quad+\int_{\Gamma} V_{\mathrm{n}} \boldsymbol{u} \cdot\left(\operatorname{div}\left(A_{\mathrm{ext}} e(\boldsymbol{u})\right)-\operatorname{div}_{\Gamma}\left(\Pi_{d}\left(A_{\mathrm{ext}} e(\boldsymbol{u})\right)\right)\right) \\
= & \int_{\Omega \backslash \bar{\omega}} \boldsymbol{f} \cdot \boldsymbol{u}^{\prime}+\int_{\Gamma_{N}} \boldsymbol{g} \cdot \boldsymbol{u}^{\prime}+\int_{\Gamma}\left(\left(A_{\mathrm{ext}} e(\boldsymbol{u}) \mathbf{n}\right) \cdot \boldsymbol{u}^{\prime}+\boldsymbol{u} \cdot\left(A_{\mathrm{ext}} e\left(\boldsymbol{u}^{\prime}\right) \mathbf{n}\right)\right) \\
& +\int_{\Gamma} V_{\mathrm{n}}\left(\partial_{\mathbf{n}} \boldsymbol{u}\right) \cdot\left(A_{\mathrm{ext}} e(\boldsymbol{u}) \mathbf{n}\right)+\int_{\Gamma} V_{\mathrm{n}}\left(\left[\nabla_{\Gamma} \boldsymbol{u}\right]: \Pi_{d}\left(A_{\mathrm{ext}} e(\boldsymbol{u})\right)\right) \\
= & \int_{\Omega \backslash \bar{\omega}} \boldsymbol{f} \cdot \boldsymbol{u}^{\prime}+\int_{\Gamma_{N}} \boldsymbol{g} \cdot \boldsymbol{u}^{\prime}+\int_{\Gamma}\left(\left(A_{\mathrm{ext}} e(\boldsymbol{u}) \mathbf{n}\right) \cdot \boldsymbol{u}^{\prime}+\boldsymbol{u} \cdot\left(A_{\mathrm{ext}} e\left(\boldsymbol{u}^{\prime}\right) \mathbf{n}\right)\right) \\
& +\int_{\Gamma} V_{\mathrm{n}}\left(e(\boldsymbol{u}): A_{\mathrm{ext}} e(\boldsymbol{u})\right)
\end{aligned}
$$

In a second step, we introduce the solution $\boldsymbol{p}$ of the adjoint problem 1.9 to spare us the computation of $\boldsymbol{u}^{\prime}$. Let set

$$
\mathcal{I}:=\int_{\Omega \backslash \bar{\omega}} \boldsymbol{f} \cdot \boldsymbol{u}^{\prime}+\int_{\Gamma_{N}} \boldsymbol{g} \cdot \boldsymbol{u}^{\prime}+\int_{\Gamma}\left(\left(A_{\mathrm{ext}} e(\boldsymbol{u}) \mathbf{n}\right) \cdot \boldsymbol{u}^{\prime}+\boldsymbol{u} \cdot\left(A_{\mathrm{ext}} e\left(\boldsymbol{u}^{\prime}\right) \mathbf{n}\right)\right) .
$$

Using the following second Green's formula (see, e.g., [4, Theorem 2.6])

$\int_{\Omega \backslash \bar{\omega}}\left(\left(\operatorname{div} A_{\mathrm{ext}} e\left(\boldsymbol{u}^{\prime}\right)\right) \cdot \boldsymbol{p}-\boldsymbol{u}^{\prime} \cdot\left(\operatorname{div} A_{\mathrm{ext}} e(\boldsymbol{p})\right)\right)=\int_{\partial(\Omega \backslash \bar{\omega})}\left(A_{\mathrm{ext}} e\left(\boldsymbol{u}^{\prime}\right) \mathbf{n}\right) \cdot \boldsymbol{p}-\int_{\partial(\Omega \backslash \bar{\omega})} \boldsymbol{u}^{\prime} \cdot\left(A_{\mathrm{ext}} e(\boldsymbol{p}) \mathbf{n}\right)$, 
and using the characterization (1.6) of $\boldsymbol{u}^{\prime}$, we get

$$
\begin{aligned}
\int_{\Omega \backslash \bar{\omega}} \boldsymbol{f} \cdot \boldsymbol{u}^{\prime} & =-\int_{\Omega \backslash \bar{\omega}}\left(\operatorname{div}\left(A_{\mathrm{ext}} e(\boldsymbol{p})\right)\right) \cdot \boldsymbol{u}^{\prime} \\
& =\int_{\partial(\Omega \backslash \bar{\omega})} \boldsymbol{p} \cdot\left(A_{\mathrm{ext}} e\left(\boldsymbol{u}^{\prime}\right) \mathbf{n}\right)-\int_{\partial(\Omega \backslash \bar{\omega})} \boldsymbol{u}^{\prime} \cdot\left(A_{\mathrm{ext}} e(\boldsymbol{p}) \mathbf{n}\right) \\
& =\int_{\Gamma} \boldsymbol{p} \cdot\left(A_{\mathrm{ext}} e\left(\boldsymbol{u}^{\prime}\right) \mathbf{n}\right)-\int_{\Gamma_{N}} \boldsymbol{u}^{\prime} \cdot \boldsymbol{g}-\int_{\Gamma} \boldsymbol{u}^{\prime} \cdot\left(A_{\mathrm{ext}} e(\boldsymbol{p}) \mathbf{n}\right) \\
& =\int_{\Gamma} \boldsymbol{p} \cdot\left(\xi\left(\boldsymbol{u}, V_{\mathrm{n}}\right)+\varepsilon \operatorname{div}_{\Gamma} \sigma_{\Gamma}\left(\boldsymbol{u}^{\prime}\right)\right)-\int_{\Gamma_{N}} \boldsymbol{u}^{\prime} \cdot \boldsymbol{g}-\int_{\Gamma} \boldsymbol{u}^{\prime} \cdot\left(A_{\mathrm{ext}} e(\boldsymbol{p}) \mathbf{n}\right) \\
& =\int_{\Gamma} \boldsymbol{p} \cdot \xi\left(\boldsymbol{u}, V_{\mathrm{n}}\right)-\int_{\Gamma_{N}} \boldsymbol{u}^{\prime} \cdot \boldsymbol{g}-\int_{\Gamma} \boldsymbol{u}^{\prime} \cdot\left(A_{\mathrm{ext}} e(\boldsymbol{p}) \mathbf{n}-\varepsilon \operatorname{div}_{\Gamma} \sigma_{\Gamma}(\boldsymbol{p})\right) .
\end{aligned}
$$

Then

$$
\begin{aligned}
\mathcal{I}= & \int_{\Gamma} \boldsymbol{p} \cdot \xi\left(\boldsymbol{u}, V_{\mathrm{n}}\right)-\int_{\Gamma} \boldsymbol{u}^{\prime} \cdot\left(A_{\mathrm{ext}} e(\boldsymbol{p}) \mathbf{n}-\varepsilon \operatorname{div}_{\Gamma} \sigma_{\Gamma}(\boldsymbol{p})\right) \\
& \quad+\int_{\Gamma}\left(\left(A_{\mathrm{ext}} e(\boldsymbol{u}) \mathbf{n}\right) \cdot \boldsymbol{u}^{\prime}+\boldsymbol{u} \cdot\left(A_{\mathrm{ext}} e\left(\boldsymbol{u}^{\prime}\right) \mathbf{n}\right)\right) \\
= & \int_{\Gamma} \boldsymbol{p} \cdot \xi\left(\boldsymbol{u}, V_{\mathrm{n}}\right)+\int_{\Gamma}\left(-\left(A_{\mathrm{ext}} e(\boldsymbol{u}) \mathbf{n}\right) \cdot \boldsymbol{u}^{\prime}+\boldsymbol{u} \cdot\left(A_{\mathrm{ext}} e\left(\boldsymbol{u}^{\prime}\right) \mathbf{n}\right)\right) \\
= & \int_{\Gamma} \boldsymbol{p} \cdot \xi\left(\boldsymbol{u}, V_{\mathrm{n}}\right)+\int_{\Gamma} \boldsymbol{u} \cdot \xi\left(\boldsymbol{u}, V_{\mathrm{n}}\right) .
\end{aligned}
$$

Finally, coming back to the cost function, we obtain

$$
\nabla \mathcal{J}(\Omega \backslash \bar{\omega}) \cdot \boldsymbol{V}=\mathcal{I}+\int_{\Gamma} V_{\mathrm{n}}\left(e(\boldsymbol{u}): A_{\mathrm{ext}} e(\boldsymbol{u})\right)=\int_{\Gamma}(\boldsymbol{p}+\boldsymbol{u}) \cdot \xi\left(\boldsymbol{u}, V_{\mathrm{n}}\right)+\int_{\Gamma} V_{\mathrm{n}}\left(e(\boldsymbol{u}): A_{\mathrm{ext}} e(\boldsymbol{u})\right) .
$$

Let set $\boldsymbol{v}:=\boldsymbol{p}+\boldsymbol{u}$. Then we use the following integration by part formula to compute the $\mathrm{L}^{2}$-adjoint operator $\xi^{*}$ with respect to the second variable of $\xi$ and write

$$
\int_{\Gamma} \boldsymbol{v} \cdot \xi\left(\boldsymbol{u}, V_{\mathrm{n}}\right)=\int_{\Gamma} V_{\mathrm{n}} \xi^{*}(\boldsymbol{u}, \boldsymbol{v})
$$

More precisely, we use the well-known results [35, Eq (5.51)]: for a scalar density $\varphi_{1}$ and a tangential vector density $\varphi_{2}$ we have

$$
-\int_{\Gamma}\left(\nabla_{\Gamma} \varphi_{1}\right) \cdot \varphi_{2}=\int_{\Gamma} \varphi_{1} \operatorname{div}_{\Gamma} \varphi_{2}
$$

Then, for every terms of $\xi(\boldsymbol{u}, \boldsymbol{v})$, we obtain successively:

$$
\begin{aligned}
\int_{\Gamma} \operatorname{div}_{\Gamma}\left(V_{\mathrm{n}} \Pi_{d}\left(A_{\mathrm{ext}} e(\boldsymbol{u})\right) \Pi_{d}\right) \cdot \boldsymbol{v} & =-\int_{\Gamma}\left(V_{\mathrm{n}} \Pi_{d}\left(A_{\mathrm{ext}} e(\boldsymbol{u})\right) \Pi_{d}\right):\left(\nabla_{\Gamma} \boldsymbol{v}\right), \\
-\int_{\Gamma} \operatorname{div}_{\Gamma}\left(V_{\mathrm{n}}\left(\left[\mathrm{D}^{2} b\right]-\mathrm{H} \Pi_{d}\right) \sigma_{\Gamma}(\boldsymbol{u})\right) \cdot \boldsymbol{v} & =\int_{\Gamma}\left(V_{\mathrm{n}}\left(\left[\mathrm{D}^{2} b\right]-\mathrm{H} \Pi_{d}\right) \sigma_{\Gamma}(\boldsymbol{u})\right):\left(\nabla_{\Gamma} \boldsymbol{v}\right), \\
-\int_{\Gamma} \operatorname{div}_{\Gamma}\left(V_{\mathrm{n}}\left(\bar{\lambda}_{\mathrm{int}}\left(\operatorname{Tr}\left[\left[\mathrm{D}^{2} b\right] \nabla_{\Gamma} \boldsymbol{u}\right]\right) \Pi_{d}\right)\right) \cdot \boldsymbol{v} & =\int_{\Gamma} V_{\mathrm{n}}\left(\left[\mathrm{D}^{2} b\right] \nabla_{\Gamma} \boldsymbol{u}\right):\left(\bar{\lambda}_{\mathrm{int}}\left(\operatorname{div}_{\Gamma} \boldsymbol{v}\right) \Pi_{d}\right) .
\end{aligned}
$$

Moreover

$$
\begin{aligned}
-\int_{\Gamma} \operatorname{div}_{\Gamma}\left(V_{\mathrm{n}}\left(2 \mu_{\mathrm{int}} \Pi_{d}\left[\frac{1}{2}\left(\left[\left[\mathrm{D}^{2} b\right] \nabla_{\Gamma} \boldsymbol{u}\right]+{ }^{t}\left[\left[\mathrm{D}^{2} b\right] \nabla_{\Gamma} \boldsymbol{u}\right]\right)\right] \Pi_{d}\right)\right) \cdot \boldsymbol{v} \\
\quad=\int_{\Gamma} V_{\mathrm{n}}\left(\left[\mathrm{D}^{2} b\right] \nabla_{\Gamma} \boldsymbol{u}\right):\left(2 \mu_{\mathrm{int}} e_{\Gamma}(\boldsymbol{v})\right)
\end{aligned}
$$


and

$$
\begin{aligned}
\int_{\Gamma} \operatorname{div}_{\Gamma}\left(\sigma_{\Gamma}\left(V_{\mathrm{n}} \partial_{\mathbf{n}} \boldsymbol{u}\right)\right) \cdot \boldsymbol{v} & =\int_{\Gamma} V_{\mathrm{n}}\left(\partial_{\mathbf{n}} \boldsymbol{u}\right) \cdot \operatorname{div}_{\Gamma}\left(\sigma_{\Gamma}(\boldsymbol{v})\right), \\
\int_{\Gamma}\left(\left[\mathrm{D}^{2} b\right]+\mathbf{n} \operatorname{div}_{\Gamma} \Pi_{d}\right)\left(\operatorname{div}_{\Gamma}\left(V_{\mathrm{n}} \sigma_{\Gamma}(\boldsymbol{u})\right)\right) \cdot \boldsymbol{v} & =\int_{\Gamma} V_{\mathrm{n}} \sigma_{\Gamma}(\boldsymbol{u}):\left(\nabla_{\Gamma}\left(\left[\nabla_{\Gamma} \boldsymbol{v}\right] \mathbf{n}\right)\right), \\
\int_{\Gamma} \operatorname{div}_{\Gamma}\left(\bar{\lambda}_{\mathrm{int}}\left(\left[\nabla_{\Gamma} \boldsymbol{u}\right] \mathbf{n} \cdot \nabla_{\Gamma} V_{\mathrm{n}}\right) \Pi_{d}\right) \cdot \boldsymbol{v} & =\int_{\Gamma} V_{\mathrm{n}} \operatorname{div}_{\Gamma}\left(\bar{\lambda}_{\mathrm{int}}\left(\operatorname{div}_{\Gamma} \boldsymbol{v}\right)\left[\nabla_{\Gamma} \boldsymbol{u}\right] \mathbf{n}\right)
\end{aligned}
$$

and finally

$$
\int_{\Gamma} \operatorname{div}_{\Gamma}\left(2 \mu_{\mathrm{int}}\left[\frac{1}{2}\left(\left[\nabla_{\Gamma} \boldsymbol{u}\right] \mathbf{n} \otimes \nabla_{\Gamma} V_{\mathrm{n}}+\nabla_{\Gamma} V_{\mathrm{n}} \otimes\left[\nabla_{\Gamma} \boldsymbol{u}\right] \mathbf{n}\right)\right]\right) \cdot \boldsymbol{v}=\int_{\Gamma} V_{\mathrm{n}} \operatorname{div}_{\Gamma}\left(2 \mu_{\mathrm{int}} e_{\Gamma}(\boldsymbol{v})\left[\nabla_{\Gamma} \boldsymbol{u}\right] \mathbf{n}\right) .
$$

We conclude by summing all the right-hand sides.

\subsection{Numerical simulations for a 2D-cantilever}

For the numerical simulations presented in this section, we consider the case where we optimize a part of the boundary of the solid which is not necessarily an inclusion. Hence we consider an elastic solid with a reference configuration $\Omega$, a bounded open set of $\mathbb{R}^{2}$, built on a part $\Gamma_{D}$ of its boundary and subjected to a load $\boldsymbol{g}$ on another part $\Gamma_{N}$. We consider here that $\Gamma:=\partial \Omega \backslash\left(\Gamma_{D} \cup \Gamma_{N}\right)$ is a nonempty set and is the only part of $\Gamma$ which can be optimized (see Figure 2). We assume that $\boldsymbol{f}=\mathbf{0}$, then the displacement $\boldsymbol{u} \in \mathcal{H}(\Omega)$ of the structure is the solution of

$$
\left\{\begin{aligned}
-\operatorname{div}\left(A_{\text {ext }} e(\boldsymbol{u})\right) & =\mathbf{0} & & \text { in } \Omega \\
\boldsymbol{u} & =\mathbf{0} & & \text { on } \Gamma_{D} \\
\left(A_{\text {ext }} e(\boldsymbol{u})\right) \mathbf{n} & =\boldsymbol{g} & & \text { on } \Gamma_{N} \\
\left(A_{\text {ext }} e(\boldsymbol{u})\right) \mathbf{n}-\varepsilon \operatorname{div}_{\Gamma}\left(\sigma_{\Gamma}(\boldsymbol{u})\right) & =\mathbf{0} & & \text { on } \Gamma .
\end{aligned}\right.
$$

We want to minimize the compliance adding a penalization on the total mass of the structure. Hence, as in the previous section, we consider the functional

$$
J(\Omega)=\mathcal{J}(\Omega)+\ell|\Omega|=\int_{\Omega} A_{\text {ext }} e(\boldsymbol{u}): e(\boldsymbol{u})+\ell|\Omega| .
$$

According to Proposition 1.4 and taking into account of the shape derivative of the volume, the shape derivative of the cost functional $J$ can be written in the form

$$
\nabla J(\Omega) \cdot \boldsymbol{V}=\int_{\Gamma} V_{\mathrm{n}}\left(\xi^{*}(\boldsymbol{u}, \boldsymbol{p}+\boldsymbol{u})+\left(A_{\mathrm{ext}} e(\boldsymbol{u}): e(\boldsymbol{u})\right)+\ell\right),
$$

where $\boldsymbol{V}$ is an admissible deformation and where $\xi^{*}$ and the adjoint state $\boldsymbol{p}$ are defined similarly as in Proposition 1.4

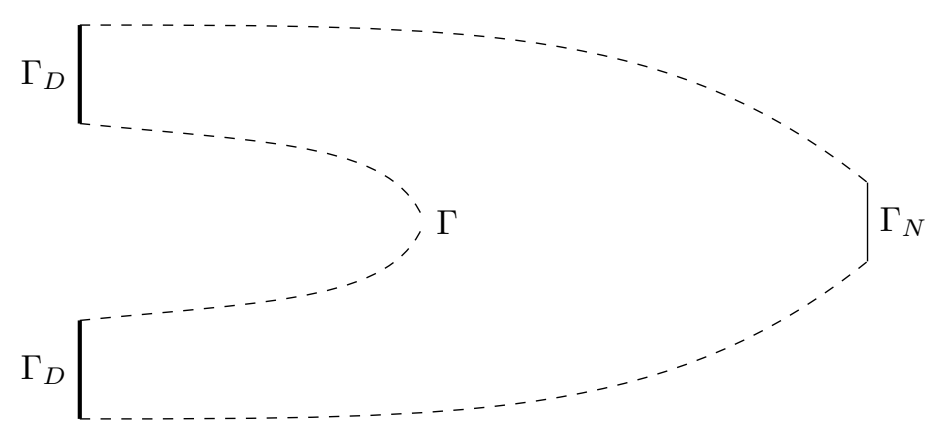

Figure 2: A cantilever.

For the numerical simulations, we use Freefem ++ (see 34) and the following parameters: $\varepsilon=0.05, \boldsymbol{g}=\left(\begin{array}{c}0 \\ -1\end{array}\right), \ell=0.05$ (the penalization of the volume), $Y=15$ (the Young Modulus), 
$\nu=0.35$ (the Poisson Ratio), $\lambda=Y * \frac{\nu}{(1 .+\nu) *(1 .-2 \cdot * \nu)}$ and $\mu=\frac{Y}{2 \cdot *(1 .+\nu)}$ (the Lamé Moduli). Moreover, we define the initial shape $\Omega_{0}$ as follows:

- $\Gamma_{D}:=\{x=0 ; y=[-1,-0.5] \cup[0.5,1]\}$

- $\Gamma_{N}:=\{x=4 ; y=[-0.1,0.1]\}$

- $\Gamma$ is given by joining the ends of the segments by straight lines,

and we define a big box $D$ as the rectangle formed by the points $(-0.5,2),(-0.5,-2),(4.5,-2)$ and $(4.5,2)$. We then follow the classical above algorithm.

\section{Algorithm}

1. We initialize the algorithm with a shape $\Omega_{0}$ contained in a big box $D$.

2. We solve the problem on the current shape $\Omega_{i}$.

3. We define $\boldsymbol{V} \in \tilde{\mathcal{U}}$, where

$$
\widetilde{\mathcal{U}}:=\left\{\boldsymbol{V}:=\left(\begin{array}{l}
\boldsymbol{V}_{x} \\
\boldsymbol{V}_{y}
\end{array}\right) \in \mathbf{H}^{1}(D) ; \boldsymbol{V}=\mathbf{0} \text { on } \Gamma_{N}, \boldsymbol{V}_{x}=0 \text { on } \Gamma_{D},\right.
$$

$\boldsymbol{V}_{x}=0$ on the left and the right side of $D, \boldsymbol{V}_{y}=0$ on the top and the bottom of $\left.D\right\}$, such that for all $\boldsymbol{W} \in \tilde{\mathcal{U}}$,

$$
\int_{D} A e(\boldsymbol{V}): e(\boldsymbol{W})+\nabla J(\Omega) \cdot \boldsymbol{W}=0 .
$$

4. We defined the convected set of $\Omega_{i}$ along the field $\boldsymbol{\theta}$ during a "small" time $\delta t$

$$
\Omega_{i+1}=\boldsymbol{X}_{i}\left(\Omega_{i}, \delta t\right)
$$

where $\boldsymbol{X}_{i}: \mathbb{R}^{2} \times \mathbb{R} \rightarrow \mathbb{R}^{2}$ is the application such that

$$
\left\{\begin{aligned}
\boldsymbol{X}_{i} & =\boldsymbol{x} & & \text { for all } \boldsymbol{x} \in \mathbb{R}^{2} \\
\frac{\partial \boldsymbol{X}_{i}}{\partial t}(\boldsymbol{x}, t) & =\boldsymbol{V}\left(\boldsymbol{X}_{i}(\boldsymbol{x}, t)\right) & & \text { for all }(\boldsymbol{x}, t) \in \mathbb{R}^{2} \times \mathbb{R} .
\end{aligned}\right.
$$

5. If $\boldsymbol{V}$ is small, we stop, else we return to the step 2.

We obtain the following result exposed in Figure 3 .

We also make a simulation adding a hole (with the generalized boundary conditions) in the initial shape. We obtain the following result exposed in Figure 4.

\section{A Well-posedness of the GIBC problem}

This section claims the well-posedness of the GIBC model (1.4) for non-vanishing boundary conditions on $\Gamma$. Hence we focus on the following problem for some given $\boldsymbol{f}, \boldsymbol{g}$ and $\boldsymbol{h}$ :

$$
\left\{\begin{aligned}
-\operatorname{div}\left(A_{\mathrm{ext}} e(\boldsymbol{u})\right) & =\boldsymbol{f} & & \text { in } \Omega \backslash \bar{\omega} \\
\boldsymbol{u} & =\mathbf{0} & & \text { on } \Gamma_{D} \\
\left(A_{\mathrm{ext}} e(\boldsymbol{u})\right) \mathbf{n} & =\boldsymbol{g} & & \text { on } \Gamma_{N} \\
\left(A_{\mathrm{ext}} e(\boldsymbol{u})\right) \mathbf{n}-\varepsilon \operatorname{div}_{\Gamma}\left(\sigma_{\Gamma}(\boldsymbol{u})\right) & =\boldsymbol{h} & & \text { on } \Gamma .
\end{aligned}\right.
$$

In the following, the dual space of $\mathbf{H}_{\Gamma_{D}}^{1}(\Omega \backslash \bar{\omega})$ is denoted by $\widetilde{\mathbf{H}}_{\Gamma_{D}}^{-1}(\Omega \backslash \bar{\omega})$.

Proposition A.1. Under the assumptions $\boldsymbol{f} \in \widetilde{\mathbf{H}}_{\Gamma_{D}}^{-1}(\Omega \backslash \bar{\omega}), \boldsymbol{g} \in \mathbf{H}^{-1 / 2}\left(\Gamma_{N}\right)$ and $\boldsymbol{h} \in \mathbf{V}^{\prime}(\Gamma)$, Problem A.1 admits a unique solution $\boldsymbol{u} \in \mathcal{H}(\Omega \backslash \bar{\omega})$. Moreover we have the following estimate, for some positive constant $C>0$,

$$
\|\boldsymbol{u}\|_{\mathcal{H}(\Omega \backslash \bar{\omega})} \leq C\left(\|\boldsymbol{f}\|_{\widetilde{\mathbf{H}}_{\Gamma_{D}}^{-1}(\Omega \backslash \bar{\omega})}+\|\boldsymbol{g}\|_{\mathbf{H}^{-1 / 2}\left(\Gamma_{N}\right)}+\|\boldsymbol{h}\|_{\mathbf{V}^{\prime}(\Gamma)}\right) .
$$



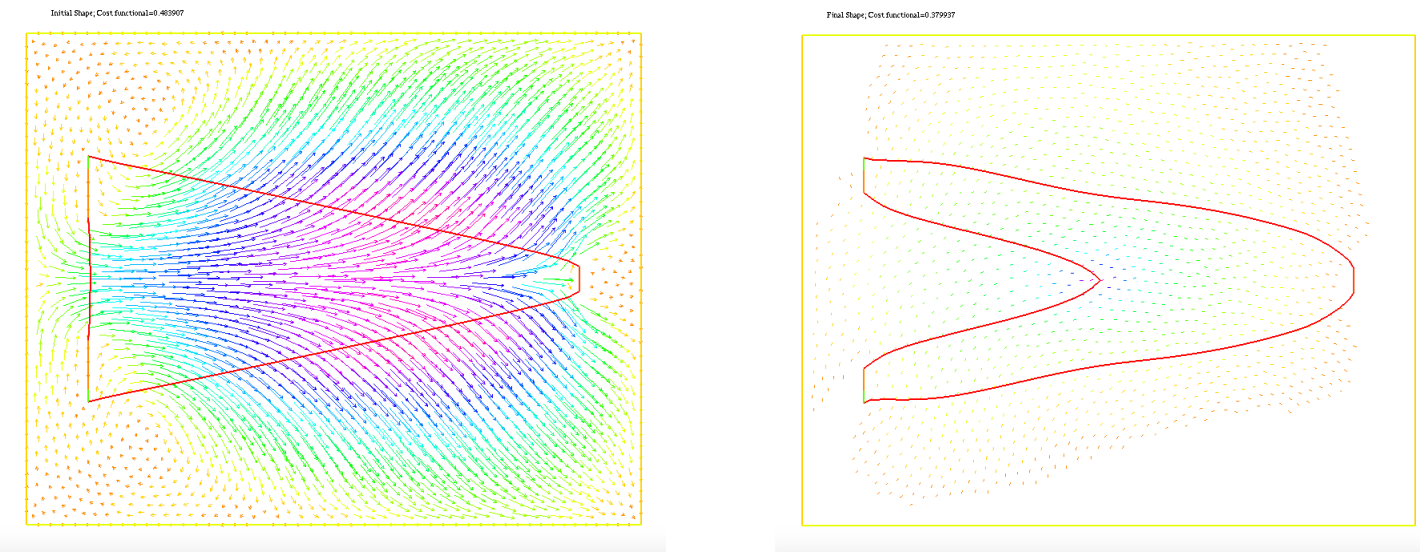

Figure 3: Minimization of the compliance for a 2d-cantilever: initial shape (left) and final shape (right).
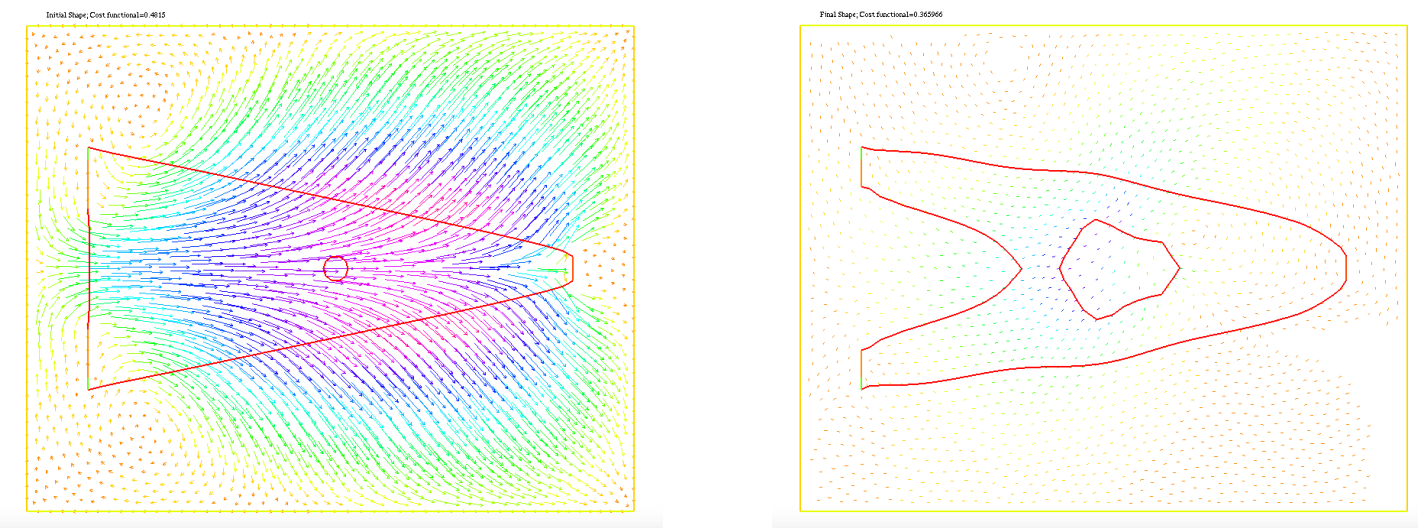

Figure 4: Minimization of the compliance for a 2d-cantilever with a hole: initial shape (left) and final shape (right).

Proof. Since $\left|\Gamma_{D}\right|>0$, we can use the second Korn's inequality (see, e.g., [20, Theorem 6.3-4]) to get the existence of a positive constant $C_{\left(\Omega \backslash \bar{\omega}, \Gamma_{D}\right)}$, which depends only on $\Omega \backslash \bar{\omega}$ and $\Gamma_{D}$, such that

$$
C_{\left(\Omega \backslash \bar{\omega}, \Gamma_{D}\right)}\|\boldsymbol{u}\|_{\mathbf{H}^{1}(\Omega \backslash \bar{\omega})} \leq\|e(\boldsymbol{u})\|_{\mathbf{L}^{2}(\Omega \backslash \bar{\omega})} \leq C_{\left(\Omega \backslash \bar{\omega}, \Gamma_{D}\right)}^{-1}\|\boldsymbol{u}\|_{\mathbf{H}^{1}(\Omega \backslash \bar{\omega})} .
$$

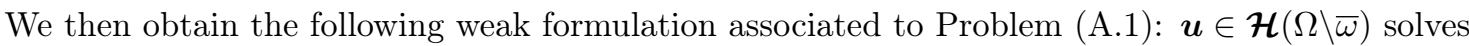

$$
\boldsymbol{a}(\boldsymbol{u}, \boldsymbol{v})=\ell(\boldsymbol{v}), \quad \forall \boldsymbol{v} \in \mathcal{H}(\Omega \backslash \bar{\omega})
$$

where

$$
\begin{aligned}
\boldsymbol{a}(\boldsymbol{u}, \boldsymbol{v}):=\lambda \int_{\Omega \backslash \bar{\omega}}(\operatorname{div} \boldsymbol{u})(\operatorname{div} \boldsymbol{v})+2 \mu \int_{\Omega \backslash \bar{\omega}} e(\boldsymbol{u}): e(\boldsymbol{v}) & \\
& +\varepsilon \bar{\lambda}_{\operatorname{int}} \int_{\Gamma}\left(\operatorname{div}_{\Gamma} \boldsymbol{u}\right)\left(\operatorname{div}_{\Gamma} \boldsymbol{v}\right)+2 \varepsilon \mu_{\mathrm{int}} \int_{\Gamma} e_{\Gamma}(\boldsymbol{u}): e_{\Gamma}(\boldsymbol{v}),
\end{aligned}
$$

and

$$
\ell(\boldsymbol{v}):=\int_{\Omega \backslash \bar{\omega}} \boldsymbol{f} \cdot \boldsymbol{v}+\int_{\Gamma_{N}} \boldsymbol{g} \cdot \boldsymbol{v}+\int_{\Gamma} \boldsymbol{h} \cdot \boldsymbol{v}
$$

Notice that the above integrals can be understood as duality products. Since the constants $\lambda, \mu$, $\bar{\lambda}_{\text {int }}$ and $\mu_{\text {int }}$ are positive, the symmetric bilinear form $\boldsymbol{a}$ is continuous and coercive on $\mathcal{H}(\Omega \backslash \bar{\omega})$. 
Indeed, since the trace operator is continuous and since $\operatorname{div} \boldsymbol{u}=\operatorname{Tr}(e(\boldsymbol{u}))$ and $\operatorname{div}_{\Gamma} \boldsymbol{u}=\operatorname{Tr}\left(e_{\Gamma}(\boldsymbol{u})\right)$, then there exist two positive constants $c_{1}$ and $c_{2}$ such that, for any $\boldsymbol{u}, \boldsymbol{v} \in \mathcal{H}(\Omega \backslash \bar{\omega})$,

$$
\begin{aligned}
|\boldsymbol{a}(\boldsymbol{u}, \boldsymbol{v})| & \leq c_{1}\|e(\boldsymbol{u})\|_{\mathbf{L}^{2}(\Omega \backslash \bar{\omega})}\|e(\boldsymbol{v})\|_{\mathbf{L}^{2}(\Omega \backslash \bar{\omega})}+c_{2} \varepsilon\left\|e_{\Gamma}(\boldsymbol{u})\right\|_{\mathbf{L}^{2}(\Gamma)}\left\|e_{\Gamma}(\boldsymbol{v})\right\|_{\mathbf{L}^{2}(\Gamma)} \\
& \leq\left(c_{1}+c_{2} \varepsilon\right) \max \left(1, C_{\left(\Omega \backslash \bar{\omega}, \Gamma_{D}\right)}^{-1}\right)\|\boldsymbol{u}\|_{\mathcal{H}(\Omega \backslash \bar{\omega})}\|\boldsymbol{v}\|_{\mathcal{H}(\Omega \backslash \bar{\omega})} .
\end{aligned}
$$

Then, there exists a positive constant $c_{3}:=\min \left(2 \mu C_{\left(\Omega \backslash \bar{\omega}, \Gamma_{D}\right)}, 2 \varepsilon \mu_{\text {int }}\right)$ such that

$$
\boldsymbol{a}(\boldsymbol{u}, \boldsymbol{u}) \geq 2 \mu\|e(\boldsymbol{u})\|_{\mathbf{L}^{2}(\Omega \backslash \bar{\omega})}^{2}+2 \varepsilon \mu_{\mathrm{int}}\left\|e_{\Gamma}(\boldsymbol{u})\right\|_{\mathbf{L}^{2}(\Gamma)}^{2} \geq c_{3}\|\boldsymbol{u}\|_{\mathcal{H}(\Omega \backslash \bar{\omega})}^{2} .
$$

The linear form $\boldsymbol{\ell}$ is also continuous on $\mathcal{H}(\Omega \backslash \bar{\omega})$. Then the Lax-Milgram theorem ensures the existence and uniqueness of the solution $\boldsymbol{u} \in \mathcal{H}(\Omega \backslash \bar{\omega})$.

\section{B Asymptotics in the thin layer}

This appendix is devoted to the derivation of the asymptotic expansion of the Lamé operator in the thin layer and the traction operator on the boundary with respect to the thickness parameter $\varepsilon \rightarrow 0$.

Recall that the thin layer coating the interior of $\Omega \backslash \bar{\omega}$ is defined by:

$$
\omega_{\text {int }}^{\varepsilon}:=\{x+s \mathbf{n}(x) \mid x \in \Gamma \text { and } 0<s<\varepsilon\} .
$$

For any smooth scalar function $u$ defined in $\omega_{\text {int }}^{\varepsilon}$ and $0<s<\varepsilon$, we set $u_{\mid \Gamma_{s}}(x+s \mathbf{n}(x))=\bar{u}(x, s)$ and, for $N \in \mathbb{N}^{*}$, we have (see [40, Eqs (2.5.182) and (2.5.208)])

$$
(\nabla u)(x+s \mathbf{n}(x))=\mathbf{n}(x) \partial_{s} \bar{u}(x, s)+\nabla_{\Gamma} \bar{u}(x, s)+\sum_{\ell=1}^{N} s^{\ell}(-1)^{\ell}\left[\mathrm{D}^{2} b\right]^{\ell} \nabla_{\Gamma} \bar{u}(x, s)+O\left(s^{N+1}\right) .
$$

We also have, for $N \in \mathbb{N}^{*}$ and for any smooth vector function $\boldsymbol{u}$ defined in $\omega_{\text {int }}^{\varepsilon}$ (see [37, Section 2]),

$$
(\operatorname{div} \boldsymbol{u})(x+s \mathbf{n}(x))=\mathbf{n}(x) \cdot \partial_{s} \overline{\boldsymbol{u}}(x, s)+\operatorname{div}_{\Gamma} \overline{\boldsymbol{u}}(x, s)+\sum_{\ell=1}^{N} s^{\ell}(-1)^{\ell} \boldsymbol{D}^{\ell} \overline{\boldsymbol{u}}(x, s)+O\left(s^{N+1}\right),
$$

with

$$
\boldsymbol{D}^{1} \overline{\boldsymbol{u}}=\operatorname{Tr}\left[\left[\mathrm{D}^{2} b\right] \nabla_{\Gamma} \overline{\boldsymbol{u}}\right]=\operatorname{div}_{\Gamma}\left(\left[\mathrm{D}^{2} b\right]-\mathrm{H}\right) \Pi_{d} \overline{\boldsymbol{u}}+\operatorname{Hdiv}_{\Gamma} \Pi_{d} \overline{\boldsymbol{u}}+\left(\mathrm{H}^{2}-2 \mathrm{G}\right)(\mathbf{n} \cdot \overline{\boldsymbol{u}}),
$$

where $\mathrm{H}=\operatorname{Tr}\left[\mathrm{D}^{2} b\right]$ is the mean curvature and $\mathrm{G}$ is the gaussian curvature. We use the following change of variable $y=x+s \mathbf{n}(x)=x+\varepsilon S \mathbf{n}(x)$ with $S \in[0 ; 1]$. We set $\overline{\boldsymbol{u}}(x, s)=\overline{\boldsymbol{u}}(x, \varepsilon S)=: \mathbf{U}(x, S)$ and we obtain the following asymptotic expansions when $\varepsilon \rightarrow 0$

$$
\begin{aligned}
(\nabla \boldsymbol{u})(x+s \mathbf{n}(x)) & =\frac{1}{\varepsilon} \mathbf{n}(x) \partial_{S} \mathbf{U}(x, S)+\nabla_{\Gamma} \mathbf{U}(x, S)+\sum_{\ell=1}^{N} \varepsilon^{\ell} S^{\ell}(-1)^{\ell}\left[\mathrm{D}^{2} b\right]^{\ell} \nabla_{\Gamma} \mathbf{U}(x, S)+O\left(\varepsilon^{N+1}\right), \\
(\operatorname{div} \boldsymbol{u})(x+s \mathbf{n}(x)) & =\frac{1}{\varepsilon} \mathbf{n}(x) \cdot \partial_{S} \mathbf{U}(x, S)+\operatorname{div}_{\Gamma} \mathbf{U}(x, S)+\sum_{\ell=1}^{N} \varepsilon^{\ell} S^{\ell}(-1)^{\ell} \boldsymbol{D}^{\ell} \mathbf{U}(x, S)+O\left(\varepsilon^{N+1}\right) .
\end{aligned}
$$

Combining the last two equalities, we obtain

$$
\begin{aligned}
A_{\text {int }} e(\boldsymbol{u})(x+\varepsilon S \mathbf{n}(x))= & \frac{1}{\varepsilon}\left(\lambda_{\mathrm{int}}\left(\mathbf{n} \cdot \partial_{S} \mathbf{U}\right) \mathrm{I}_{d}+\mu_{\mathrm{int}}\left(\mathbf{n} \otimes \partial_{S} \mathbf{U}+\partial_{S} \mathbf{U} \otimes \mathbf{n}\right)\right) \\
& +\left(\lambda_{\mathrm{int}}\left(\operatorname{div}_{\Gamma} \mathbf{U}\right) \mathrm{I}_{d}+\mu_{\mathrm{int}}\left(\left[\nabla_{\Gamma} \mathbf{U}\right]+{ }^{t}\left[\nabla_{\Gamma} \mathbf{U}\right]\right)\right) \\
& +\sum_{\ell \geq 1}(-1)^{\ell} \varepsilon^{\ell} S^{\ell}\left(\lambda_{\mathrm{int}}\left(\boldsymbol{D}^{\ell} \mathbf{U}\right) \mathrm{I}_{d}+\mu_{\mathrm{int}}\left(\left[\left[\mathrm{D}^{2} b\right]^{\ell} \nabla_{\Gamma} \mathbf{U}\right]+{ }^{t}\left[\left[\mathrm{D}^{2} b\right]^{\ell} \nabla_{\Gamma} \mathbf{U}\right]\right)\right) .
\end{aligned}
$$

Using the following formulas

$\left[\nabla_{\Gamma} \mathbf{U}\right] \mathbf{n}=\nabla_{\Gamma}(\mathbf{n} \cdot \mathbf{U})-\left[\mathrm{D}^{2} b\right] \mathbf{U}, \quad \operatorname{div}_{\Gamma}(\mathbf{n} \otimes \mathbf{U})=\mathrm{HU}, \quad \operatorname{div}_{\Gamma}(\mathbf{U} \otimes \mathbf{n})=\mathbf{n} \operatorname{div}_{\Gamma} \mathbf{U}+\left[\mathrm{D}^{2} b\right] \mathbf{U}$, $\operatorname{Tr}\left[\left[\mathrm{D}^{2} b\right] \nabla_{\Gamma}(\mathbf{n} \otimes \mathbf{U})\right]=\operatorname{Tr}\left[\left[\mathrm{D}^{2} b\right]^{2}\right] \mathbf{U}, \quad$ and $\quad \operatorname{Tr}\left[\left[\mathrm{D}^{2} b\right] \nabla_{\Gamma}(\mathbf{U} \otimes \mathbf{n})\right]=\mathbf{n} \operatorname{Tr}\left[\left[\mathrm{D}^{2} b\right] \nabla_{\Gamma} \mathbf{U}\right]+\left[\mathrm{D}^{2} b\right]^{2} \mathbf{U}$, 
) we finally obtain 2.2 . Notice that we have

$$
\begin{aligned}
\Lambda_{2,2} \mathbf{U} & =\operatorname{div}_{\Gamma}\left(\lambda_{\mathrm{int}}\left(\operatorname{div}_{\Gamma} \mathbf{U}\right) \mathrm{I}_{d}+\mu_{\mathrm{int}}\left(\left[\nabla_{\Gamma} \mathbf{U}\right]+{ }^{t}\left[\nabla_{\Gamma} \mathbf{U}\right]\right)\right)-\lambda_{\mathrm{int}} \mathbf{n}\left(\boldsymbol{D}^{1} \mathbf{U}\right)-\mu_{\mathrm{int}}\left[\left[\mathrm{D}^{2} b\right] \nabla_{\Gamma} \mathbf{U}\right] \mathbf{n} \\
& =\left(\lambda_{\mathrm{int}}+\mu_{\mathrm{int}}\right) \nabla_{\Gamma} \operatorname{div}_{\Gamma} \mathbf{U}+\mu_{\mathrm{int}} \Delta_{\Gamma} \mathbf{U}-\lambda_{\mathrm{int}} \mathbf{n} \operatorname{Tr}\left[\left[\mathrm{D}^{2} b\right] \nabla_{\Gamma} \mathbf{U}\right]-\mu_{\mathrm{int}}\left[\left[\mathrm{D}^{2} b\right] \nabla_{\Gamma} \mathbf{U}\right] \mathbf{n}, \\
\Lambda_{2,1} \mathbf{V} & =-\lambda_{\mathrm{int}} \mathbf{n}\left(\boldsymbol{D}^{1} \mathbf{V}\right)-\mu_{\mathrm{int}}\left[\left[\mathrm{D}^{2} b\right] \nabla_{\Gamma} \mathbf{V}\right] \mathbf{n}-\boldsymbol{D}^{1}\left(\lambda_{\mathrm{int}}(\mathbf{n} \cdot \mathbf{V}) \mathrm{I}_{d}+\mu_{\mathrm{int}}(\mathbf{n} \otimes \mathbf{V}+\mathbf{V} \otimes \mathbf{n})\right) \\
& =-\mu_{\mathrm{int}} \operatorname{Tr}\left[\left[\mathrm{D}^{2} b\right]^{2}\right] \mathbf{V}-\left(\lambda_{\mathrm{int}}+\mu_{\mathrm{int}}\right)\left(\mathbf{n}\left(\operatorname{Tr}\left[\left[\mathrm{D}^{2} b\right] \nabla_{\Gamma} \mathbf{V}\right]\right)+\left[\mathrm{D}^{2} b\right] \nabla_{\Gamma}(\mathbf{n} \cdot \mathbf{V})\right) .
\end{aligned}
$$

Moreover the traction trace operator defined on the surface $\Gamma_{S}:=\{x+\varepsilon S \mathbf{n}(x) \mid x \in \Gamma\}$ is given, for any $0 \leq S \leq 1$, by

$$
A_{\text {int }} e(\mathbf{U})=\frac{1}{\varepsilon} \Lambda_{0} \partial_{S} \mathbf{U}+\mathbf{B}_{t}^{0} \mathbf{U}+\sum_{\ell \geq 1} \varepsilon^{\ell} S^{\ell} \mathbf{B}_{t}^{\ell} \mathbf{U}
$$

where $\mathbf{B}_{t}^{0} \mathbf{U}:=\lambda_{\text {int }} \mathbf{n} \operatorname{div}_{\Gamma} \mathbf{U}+\mu_{\mathrm{int}}\left[\nabla_{\Gamma} \mathbf{U}\right] \mathbf{n}$ and for $\ell \geq 1$,

$$
\mathbf{B}_{t}^{\ell} \mathbf{U}:=(-1)^{\ell}\left(\lambda_{\mathrm{int}} \mathbf{n}\left(\boldsymbol{D}^{\ell} \mathbf{U}\right)+\mu_{\mathrm{int}}\left[\left[\mathrm{D}^{2} b\right]^{\ell} \nabla_{\Gamma} \mathbf{U}\right] \mathbf{n}\right) .
$$

\section{References}

[1] Y. Achdou, O. Pironneau, and F. Valentin. Effective boundary conditions for laminar flows over periodic rough boundaries. J. Comput. Phys., 147(1):187-218, 1998.

[2] R. A. Adams and J. J. F. Fournier. Sobolev spaces, volume 140 of Pure and Applied Mathematics (Amsterdam). Elsevier/Academic Press, Amsterdam, second edition, 2003.

[3] G. Allaire. Shape optimization by the homogenization method, volume 146 of Applied Mathematical Sciences. Springer-Verlag, New York, 2002.

[4] G. Allaire. Conception optimale de structures, volume 58 of Mathématiques 83 Applications (Berlin) [Mathematics \& Applications]. Springer-Verlag, Berlin, 2007. With the collaboration of Marc Schoenauer (INRIA) in the writing of Chapter 8.

[5] G. Allaire, E. Bonnetier, G. Francfort, and F. Jouve. Shape optimization by the homogenization method. Numer. Math., 76(1):27-68, 1997.

[6] G. Allaire and C. Dapogny. A deterministic approximation method in shape optimization under random uncertainties. SMAI-Journal of computational mathematics, 1:83-143, 2015.

[7] G. Allaire, F. Jouve, and A.-M. Toader. Structural optimization using sensitivity analysis and a level-set method. J. Comput. Phys., 194(1):363-393, 2004.

[8] S. Amstutz and M. Ciligot-Travain. A notion of compliance robustness in topology optimization. ESAIM Control Optim. Calc. Var., 22(1):64-87, 2016.

[9] X. Antoine and H. Barucq. Approximation by generalized impedance boundary conditions of a transmission problem in acoustic scattering. M2AN Math. Model. Numer. Anal., 39(5):1041$1059,2005$.

[10] A. Bendali and K. Lemrabet. The effect of a thin coating on the scattering of a time-harmonic wave for the Helmholtz equation. SIAM J. Appl. Math., 56(6):1664-1693, 1996.

[11] M. Bonnet, A. Burel, M. Duruflé, and P. Joly. Effective transmission conditions for thinlayer transmission problems in elastodynamics. The case of a planar layer model. ESAIM: Mathematical Modelling and Numerical Analysis, 50:43-75, 2016.

[12] L. Bourgeois, N. Chaulet, and H. Haddar. On simultaneous identification of the shape and generalized impedance boundary condition in obstacle scattering. SIAM J. Sci. Comput., 34(3):A1824-A1848, 2012.

[13] H. Brezis. Functional analysis, Sobolev spaces and partial differential equations. Universitext. Springer, New York, 2011. 
[14] F. Cakoni, Y. Hu, and R. Kress. Simultaneous reconstruction of shape and generalized impedance functions in electrostatic imaging. Inverse Problems, 30(10):105009, 19, 2014.

[15] F. Cakoni and R. Kress. Integral equation methods for the inverse obstacle problem with generalized impedance boundary condition. Inverse Problems, 29(1):015005, 19, 2013.

[16] G. Caloz, M. Costabel, M. Dauge, and G. Vial. Asymptotic expansion of the solution of an interface problem in a polygonal domain with thin layer. Asymptot. Anal., 50(1-2):121-173, 2006.

[17] F. Caubet, M. Dambrine, and D. Kateb. Shape optimization methods for the inverse obstacle problem with generalized impedance boundary conditions. Inverse Problems, 29(11):115011, 26, 2013.

[18] F. Caubet, H. Haddar, J.-R. Li, and V. N. D. New transmission condition accounting for diffusion anisotropy in thin layers applied to diffusion MRI. M2AN Math. Model. Numer. Anal., 2016/17.

[19] N. Chaulet and H. Haddar. Electromagnetic inverse shape problem for coated obstacles. Adv. Comput. Math., 41(6):1179-1205, 2015.

[20] P. G. Ciarlet. Mathematical elasticity. Vol. I, volume 20 of Studies in Mathematics and its Applications. North-Holland Publishing Co., Amsterdam, 1988. Three-dimensional elasticity.

[21] P. G. Ciarlet. Mathematical elasticity. Vol. III, volume 29 of Studies in Mathematics and its Applications. North-Holland Publishing Co., Amsterdam, 2000. Theory of shells.

[22] M. Costabel and F. Le Louër. Shape derivatives of boundary integral operators in electromagnetic scattering. Part I: Shape differentiability of pseudo-homogeneous boundary integral operators. Integral Equations Operator Theory, 72(4):509-535, 2012.

[23] M. Dambrine, I. Greff, H. Harbrecht, and B. Puig. Numerical solution of the homogeneous Neumann boundary value problem on domains with a thin layer of random thickness. $J$. Comput. Phys., 330:943-959, 2017.

[24] M. Dambrine and A. Laurain. A first order approach for worst-case shape optimization of the compliance for a mixture in the low contrast regime. Struct. Multidiscip. Optim., 54(2):215231, 2016.

[25] B. Engquist and A. Majda. Absorbing boundary conditions for the numerical simulation of waves. Math. Comp., 31(139):629-651, 1977.

[26] E. Faou. Elasticity on a thin shell: formal series solution. Asymptot. Anal., 31(3-4):317-361, 2002.

[27] Z. M. Gao, Y. C. Ma, and H. W. Zhuang. Shape optimization for Navier-Stokes flow. Inverse Probl. Sci. Eng., 16(5):583-616, 2008.

[28] S. Garreau, P. Guillaume, and M. Masmoudi. The topological asymptotic for PDE systems: the elasticity case. SIAM J. Control Optim., 39(6):1756-1778, 2001.

[29] D. Givoli. Nonreflecting boundary conditions. J. Comput. Phys., 94(1):1-29, 1991.

[30] H. Haddar and P. Joly. Effective boundary conditions for thin ferromagnetic layers: the one-dimensional model. SIAM J. Appl. Math., 61(4):1386-1417, 2000/01.

[31] H. Haddar, P. Joly, and H.-M. Nguyen. Generalized impedance boundary conditions for scattering by strongly absorbing obstacles: the scalar case. Math. Models Methods Appl. Sci., 15(8):1273-1300, 2005.

[32] H. Haddar, P. Joly, and H.-M. Nguyen. Generalized impedance boundary conditions for scattering problems from strongly absorbing obstacles: the case of Maxwell's equations. Math. Models Methods Appl. Sci., 18(10):1787-1827, 2008. 
[33] J. Haslinger and R. A. E. Mäkinen. Introduction to shape optimization, volume 7 of Advances in Design and Control. Society for Industrial and Applied Mathematics (SIAM), Philadelphia, PA, 2003. Theory, approximation, and computation.

[34] F. Hecht. New development in freefem++. J. Numer. Math., 20(3-4):251-265, 2012.

[35] A. Henrot and M. Pierre. Variation et optimisation de formes, volume 48 of Mathématiques \& Applications (Berlin) [Mathematics \& Applications]. Springer, Berlin, 2005. Une analyse géométrique. [A geometric analysis].

[36] W. Jäger and A. Mikelić. On the roughness-induced effective boundary conditions for an incompressible viscous flow. J. Differential Equations, 170(1):96-122, 2001.

[37] D. Kateb and F. Le Louër. Generalized impedance boundary conditions and shape derivatives for 3D Helmholtz problems. Math. Models Methods Appl. Sci., 26(10):1995-2033, 2016.

[38] F. Le Louër. A domain derivative-based method for solving elastodynamic inverse obstacle scattering problems. Inverse Problems, 31(11):115006, 27, 2015.

[39] F. Murat and J. Simon. Sur le contrôle par un domaine géométrique. Rapport du L.A. 189, 1976. Université de Paris VI, France.

[40] J.-C. Nédélec. Acoustic and electromagnetic equations, volume 144 of Applied Mathematical Sciences. Springer-Verlag, New York, 2001. Integral representations for harmonic problems.

[41] A. A. Novotny, R. A. Feijóo, E. Taroco, and C. Padra. Topological sensitivity analysis for three-dimensional linear elasticity problem. Comput. Methods Appl. Mech. Engrg., 196(4144):4354-4364, 2007.

[42] A. A. Novotny and J. Sokołowski. Topological derivatives in shape optimization. Interaction of Mechanics and Mathematics. Springer, Heidelberg, 2013.

[43] J. r. Něcas. Direct methods in the theory of elliptic equations. Springer Monographs in Mathematics. Springer, Heidelberg, 2012. Translated from the 1967 French original by Gerard Tronel and Alois Kufner, Editorial coordination and preface by Šárka Nečasová and a contribution by Christian G. Simader.

[44] C. Poignard. Generalized impedance boundary condition at high frequency for a domain with thin layer: the circular case. Appl. Anal., 86(12):1549-1568, 2007.

[45] J. Sokołowski and J.-P. Zolésio. Introduction to shape optimization, volume 16 of Springer Series in Computational Mathematics. Springer-Verlag, Berlin, 1992. Shape sensitivity analysis.

[46] G. Vial. Analyse multi-échelle et conditions aux limites approchées pour un problème avec couche mince dans un domaine à coin. PhD thesis, Université de Rennes 1, 2003. 